\title{
Transferring Instantly the State of Higher-Order Linear Descriptor (Regular) Differential Systems Using Impulsive Inputs
}

\author{
Athanasios D. Karageorgos, ${ }^{1}$ Athanasios A. Pantelous, ${ }^{1,2,3}$ and Grigoris I. Kalogeropoulos ${ }^{1}$ \\ ${ }^{1}$ Department of Mathematics, University of Athens, Athens, Greece \\ ${ }^{2}$ School of Engineering and Mathematical Sciences, City University, London, UK \\ ${ }^{3}$ Department of Mathematical Sciences, University of Liverpool, Liverpool, UK
}

Correspondence should be addressed to Athanasios A. Pantelous, a.pantelous@liverpool.ac.uk

Received 4 March 2009; Accepted 19 June 2009

Recommended by Amit Bhaya

In many applications, and generally speaking in many dynamical differential systems, the problem of transferring the initial state of the system to a desired state in (almost) zero-time time is desirable but difficult to achieve. Theoretically, this can be achieved by using a linear combination of Dirac $\delta$-function and its derivatives. Obviously, such an input is physically unrealizable. However, we can think of it approximately as a combination of small pulses of very high magnitude and infinitely small duration. In this paper, the approximation process of the distributional behaviour of higher-order linear descriptor (regular) differential systems is presented. Thus, new analytical formulae based on linear algebra methods and generalized inverses theory are provided. Our approach is quite general and some significant conditions are derived. Finally, a numerical example is presented and discussed.

Copyright (C) 2009 Athanasios D. Karageorgos et al. This is an open access article distributed under the Creative Commons Attribution License, which permits unrestricted use, distribution, and reproduction in any medium, provided the original work is properly cited.

\section{Introduction}

From the point of view of several important applications, in several fields of research, see for instance $[1,2]$, taking a given state of a linear system to a desired state in minimum time is very desirable, though it is a challenging problem in control and system theory.

Significant attention has been given to this problem in the case of linear systems; see [1-3]. Recently, Kalogeropoulos et al. [4] have further enriched these first approaches, as they have relaxed some of the rather restricted assumptions that $[1,2]$ are considered. Afterwards, the method has been also applied to the more general class of linear descriptor (regular) systems; see [5].

In this paper, a further extension of [5], in the class of linear descriptor (regular) equations, is provided. Comparing with the existing literature, see [1-5], we solve this problem

(1) for higher-order linear differential descriptor (regular) systems (compare with [5]),

(2) using higher-order consistent initial conditions (compare with [1-5]),
(3) obtaining more analytical formulas, that is, see Appendices A and B, Theorems 20 and 23. (compare with [1-5]),

(4) without using the controllability matrix (compare with [3]),

(5) applying analytical methods for the exact determination of the generalized inverses of Vandermonde and relative to this matrices; see [6].

To summarize, in this paper, we investigate how we can transfer the initial state of an open loop, linear higherorder descriptor (regular) differential system in (practically speaking, almost) zero-time, that is,

$$
F \underline{x}^{(r)}(t)=G \underline{x}(t)+\underline{b} u(t)
$$

with known initial conditions

$$
\underline{x}\left(t_{0}\right), \underline{x}^{\prime}\left(t_{o}\right), \ldots, \underline{x}^{(r-1)}\left(t_{0}\right)
$$


where $F, G \in \mathcal{M}(n \times n ; \mathbb{F})$, and $\underline{b} \in \mathcal{M}(n \times 1 ; \mathbb{F})$ (i.e., $\mathcal{M}$ is the algebra of $n \times m$ matrices with elements in the field $\mathbb{F})$ with $\operatorname{det} M=0(0$ is the zero element of $\mathcal{M}(n=1, \mathbb{F}))$, $\underline{x}(t) \in \mathcal{C}^{\infty}(\mathbb{F}, \mathcal{M}(n \times 1 ; \mathbb{F}))$, and $u(t) \in \mathcal{C}^{\infty}(\mathbb{F}, \mathcal{M}(1 ; \mathbb{F}))$. For the sake of simplicity, we set in the sequel $\mathcal{M}_{n}=\mathcal{M}(n \times n ; \mathbb{F})$ and $\mathcal{M}_{n m}=\mathcal{M}(n \times m ; \mathbb{F})$.

In order to solve this problem, the appropriate input vector has to be made up as a linear combination of the $\delta$-function of Dirac and its derivatives, see $[1,2]$, and for more details consult [7]. Obviously, such an input is very hard to imagine physically. However, we can think of it approximately as a combination of small pulses of very high magnitude and infinitely small duration.

Linear descriptor (singular or regular) differential systems have been extensively used in control theory; see for instance [8-10], and for more details [11].

A brief outline of the paper is as follows. Section 2 provides the incentives and the typical modelling features of the problem. Moreover, a classical approximated expression for the controller, that is, a linear combination of the $\delta$ function of Dirac and its derivatives that is based on the normal (Gaussian) probability density function, is used. Then, the need to determine the unknown coefficients is derived. Section 3 is divided into four extensive subsections. In Section 3.1, the reduction of the higher-order system to first-order is discussed. The first-order descriptor (regular) is divided into a fast and a slow subsystem, using the Weierstrass canonical form. Section 3.2 investigates and presents some analytical formulas based on the slow subsystem. In Section 3.3, the theory of the $\{1\}$-generalized inverses is used. Finally, some significant conditions for the solution of the slow subsystem are presented in Section 3.4. In Section 4, a necessary condition based on the fast subsystem is discussed and obtained. Section 5 provides an interesting numerical application from physics and Section 6 concludes the paper. Two appendices for the analytical calculations of two important integrals are also available.

\section{Preliminary Results-Matrix Pencil Framework}

In this section, some preliminary results for matrix pencil and system theory are briefly presented. First, we assume that for an $n$ th-order linear differential system, see (1), the input can be a linear combination of Dirac $\delta$-function and its first $(n-1)$ th-derivatives as follows:

$$
\begin{aligned}
u_{o}(t) & =a_{o} \delta(t)+a_{1} \delta^{(1)}(t)+a_{2} \delta^{(2)}(t)+\cdots+a_{n-1} \delta^{(n-1)}(t) \\
& =\sum_{k=1}^{n-1} a_{k} \delta^{(k)}(t),
\end{aligned}
$$

where $\delta^{(k)}(t)$ or $d^{k} \delta(t) / d t^{k}$ is the $k$ th-derivative of the Dirac $\delta$-function, and $a_{i}$ for $i=0,1, \ldots, n-1$ are the magnitudes of the delta function and its derivatives. Furthermore, we assume that the state of the system at time $0^{-}$is

$$
\underline{x}\left(0^{-}\right)=\underline{x}^{\prime}\left(0^{-}\right)=\cdots \underline{x}^{(r-1)}\left(0^{-}\right)=\left[\begin{array}{llll}
0 & 0 & \cdots & 0
\end{array}\right]^{t},
$$

and at time $0^{+}$, it achieves

$$
\begin{gathered}
\underline{x}\left(0^{+}\right)=\left[\begin{array}{llll}
x_{1}^{0} & x_{2}^{0} & \cdots & x_{n}^{0}
\end{array}\right]^{t}, \quad \underline{x}^{\prime}\left(0^{+}\right)=\left[\begin{array}{llll}
x_{1}^{1} & x_{2}^{1} & \cdots & x_{n}^{1}
\end{array}\right]^{t} \\
, \ldots, \underline{x}^{(r-1)}\left(0^{+}\right)=\left[\begin{array}{llll}
x_{1}^{r-1} & x_{2}^{r-1} & \cdots & x_{n}^{r-1}
\end{array}\right]^{t} .
\end{gathered}
$$

With the following definitions, a brief presentation of the most important elements of matrix pencil theory is given.

Definition 1. Given $F, G \in \mathcal{M}_{n m}$ and an indeterminate $s \in \mathbb{F}$, the matrix pencil $s F-G$ is called regular when $m=n$ and $\operatorname{det}(s F-G) \neq 0$. In any other case, the pencil will be called singular.

Definition 2. The pencil $s F-G$ is said to be strictly equivalent to the pencil $s \widetilde{F}-\tilde{G}$ if and only if there exist nonsingular $P \in \mathcal{M}_{m}$ and $Q \in \mathcal{M}_{n}$ such as

$$
P(s F-G) Q=s \widetilde{F}-\widetilde{G} .
$$

In this paper, we consider the case that pencil is regular. Thus, the strict equivalence relation can be defined rigorously on the set of regular pencils as follows.

This is the set of elementary divisors (e.d.s) obtained by factorizing the invariant polynomials $f_{i}(s, \hat{s})$ into powers of homogeneous polynomials irreducible over field $\mathbb{F}$.

In the case where $s F-G$ is a regular, we have e.d. of the following types:

(i) e.d. of the type $s^{p}$ are called zero finite elementary divisors (z. f.e.d.)

(ii) e.d. of the type $(s-a)^{\pi}, a \neq 0$ are called nonzero finite elementary divisors (nz. f.e.d.),

(iii) e.d. of the type $\hat{s}^{q}$ are called infinite elementary divisors (i.e.d.).

Let $B_{1}, B_{2}, \ldots, B_{n}$ be elements of $\mathcal{M}_{n}$. The direct sum of them denoted by $B_{1} \oplus B_{2} \oplus \cdots \oplus B_{n}$ is the block diag $\left\{B_{1}, B_{2}, \ldots, B_{n}\right\}$.

Then, the complex Weierstrass form $s F_{w}-Q_{w}$ of the regular pencil $s F-G$ is defined by

$$
s F_{w}-Q_{w} \triangleq s I_{p}-J_{p} \bigoplus s H_{q}-I_{q} .
$$

Now, the Jordan type element, that is, $J_{p}$, is uniquely defined by the set of f.e.d.

$$
\left(s-a_{1}\right)^{p_{1}}, \ldots,\left(s-a_{v}\right)^{p_{v}}, \sum_{j=1}^{v} p_{j}=p,
$$

of $s F-G$ and has the form

$$
s I_{p}-J_{p} \triangleq s I_{p_{1}}-J_{p_{1}}\left(a_{1}\right) \bigoplus \cdots \bigoplus s I_{p_{v}}-J_{p_{v}}\left(a_{v}\right),
$$

where

$$
\begin{gathered}
I_{p} \triangleq I_{p_{1}} \bigoplus I_{p_{2}} \bigoplus \cdots \bigoplus I_{p_{v}}, \\
J_{p} \triangleq J_{p_{1}}\left(a_{1}\right) \bigoplus J_{p_{2}}\left(a_{2}\right) \bigoplus \cdots \bigoplus J_{p_{v}}\left(a_{\nu}\right) .
\end{gathered}
$$


The $q$ blocks of the second, that is, $s H_{q}-I_{q}$, see (7), are uniquely defined by the set of i.e.d.

$$
\hat{s}^{q_{1}}, \ldots, \hat{s}^{q_{\sigma}}, \sum_{j=1}^{\sigma} q_{j}=q
$$

of $s F-G$ and has the form

$$
s H_{q}-I_{q} \triangleq s H_{q_{1}}-I_{q_{1}} \bigoplus \cdots \bigoplus s H_{q_{\sigma}}-I_{q_{\sigma}},
$$

where

$$
\begin{gathered}
I_{q} \triangleq I_{q_{1}} \bigoplus I_{q_{2}} \bigoplus \cdots \oplus I_{q_{\sigma}}, \\
H_{q} \triangleq H_{q_{1}} \bigoplus H_{q_{2}} \bigoplus \cdots \oplus H_{q_{\sigma}} .
\end{gathered}
$$

Furthermore, $H_{q}$ is a nilpotent element of $\mathcal{M}_{n}$ with index $\tilde{q}=$ $\max \left\{q_{j}: j=1,2, \ldots, \sigma\right\}$, where

$$
H_{q}^{\tilde{q}}=\mathbb{O},
$$

and $I_{p_{j}}, J_{p_{j}}\left(a_{j}\right), H_{q_{j}}$ are defined as

$$
\begin{aligned}
I_{p_{j}} & =\left[\begin{array}{cccc}
1 & 0 & \cdots & 0 \\
0 & 1 & \cdots & 0 \\
\vdots & \vdots & \ddots & \vdots \\
0 & 0 & \cdots & 1
\end{array}\right] \in \mathcal{M}_{p_{j}}, \\
J_{p_{j}}\left(a_{j}\right) & =\left[\begin{array}{ccccc}
a_{j} & 1 & 0 & \cdots & 0 \\
0 & a_{j} & 1 & \cdots & 0 \\
\vdots & \vdots & \ddots & \vdots & \vdots \\
0 & 0 & 0 & a_{j} & 1 \\
0 & 0 & 0 & 0 & a_{j}
\end{array}\right] \in \mathcal{M}_{p_{j}}, \\
H_{q_{j}} & =\left[\begin{array}{lllll}
0 & 1 & 0 & \cdots & 0 \\
0 & 0 & 1 & \cdots & 0 \\
\vdots & \vdots & \ddots & \vdots & \vdots \\
0 & 0 & 0 & 0 & 1 \\
0 & 0 & 0 & 0 & 0
\end{array}\right] \in \mathcal{M}_{q_{j}} .
\end{aligned}
$$

Moreover, for the matrices $F$ and $G$, we have the parameterization

$$
\begin{aligned}
& P F Q=F_{w}=I_{p} \bigoplus H_{q}, \\
& P G Q=G_{w}=J_{p} \bigoplus I_{q} .
\end{aligned}
$$

Since the state $x\left(0^{+}\right)$which we wish to reach is specified, we need to determine the $n$ unknown coefficients $a_{i}$, for $i=0,1, \ldots, n-1$.

In practice, we cannot create an exact impulse function nor its derivatives. However, if we use one of the approximations of Dirac $\delta$-function, we will be able to change the state in some minimum practical time depending mainly upon how well we generate the approximations. Let the Dirac $\delta$ function be viewed as the limit of sequence function

$$
\delta(t)=\lim _{a \rightarrow 0} \delta_{a}(t),
$$

where $\delta_{a}(t)$ is called a nascent delta function. This limit is in the sense that

$$
\lim _{a \rightarrow 0} \int_{-\infty}^{+\infty} \delta_{a}(t) f(t) d t=f(0) .
$$

Some wellknown and very useful in applications nascent delta functions are the Normal and Cauchy distributions, the rectangular function, the derivative of the sigmoid (or Fermi-Dirac) function, the Airy function, and so forth; see for instance $[2,5,12-17]$. The results given below are based on the normal function. Thus, by taking into consideration expression (18) and the normal (Gaussian) probability density distribution, we obtain

$$
\delta(t)=\lim _{\sigma \rightarrow 0} \frac{1}{\sigma \sqrt{2 \pi}} e^{-t^{2} / 2 \sigma^{2}}=\lim _{\sigma \rightarrow 0} \frac{1}{\sigma} \Phi\left(\frac{t}{\sigma}\right),
$$

where $\Phi(x)=(1 / \sqrt{2 \pi}) e^{-x^{2} / 2}$.

So, the approximate expression for the controller (2) is given by

$$
\begin{aligned}
u(t)= & \frac{1}{\sigma} \Phi\left(\frac{t}{\sigma}\right) a_{o}+\frac{1}{\sigma^{2}} \Phi^{(1)}\left(\frac{t}{\sigma}\right) a_{1} \\
& +\cdots+\frac{1}{\sigma^{n}} \Phi^{(n-1)}\left(\frac{t}{\sigma}\right) a_{n-1} \\
= & \sum_{k=0}^{n-1} \frac{1}{\sigma^{k+1}} \Phi^{(k)}\left(\frac{t}{\sigma}\right) a_{k} .
\end{aligned}
$$

Then, we take the limit

$$
u_{o}(t)=\lim _{\sigma \rightarrow 0} u(t) .
$$

In the next section, the main results are presented.

\section{The Main Results}

3.1. Order Reduction of System (1). The section begins with the following important lemma.

Lemma 3. System (1) is divided into the following two subsystems:

$$
\begin{aligned}
& \underline{y}_{p}^{(r)}(t)=J_{p} \underline{y}_{p}(t)+\underline{\tilde{b}}_{p} u_{o}(t), \\
& H_{q} \underline{y}_{q}^{(r)}(t)=\underline{y}_{q}(t)+\underline{\tilde{b}}_{q} u_{o}(t) .
\end{aligned}
$$

Proof. Consider the transformation

$$
\underline{x}(t)=Q \underline{y}(t) .
$$

Substituting the previous expression into (1) and considering also (3), we obtain

$$
F Q \underline{y}^{(r)}(t)=G Q \underline{y}(t)+\underline{b} u_{o}(t) .
$$


Multiplying by $P$, we arrive at

$$
F_{w} \underline{y}^{(r)}(t)=G_{w} \underline{y}(t)+P \underline{b} u_{o}(t) .
$$

Now, we denote

$$
\begin{aligned}
& \underline{y}(t)=\left[\begin{array}{l}
\underline{y}_{p}(t) \\
\underline{y}_{q}(t)
\end{array}\right], \\
& P \underline{b} \triangleq \underline{\tilde{b}}=\left[\begin{array}{l}
\underline{\tilde{b}}_{p} \\
\tilde{b}_{q}
\end{array}\right],
\end{aligned}
$$

where $p+q=n$.

Taking into account the following expressions, that is,

$$
\begin{gathered}
{\left[\begin{array}{cc}
I_{p} & \mathbb{O}_{p, q} \\
\mathbb{O}_{q, p} & H_{q}
\end{array}\right]\left[\begin{array}{l}
\underline{y}_{p}^{(r)}(t) \\
\underline{y}_{q}^{(r)}(t)
\end{array}\right]} \\
=\left[\begin{array}{cc}
J_{p} & \mathbb{O}_{p, q} \\
\mathbb{O}_{q, p} & I_{q}
\end{array}\right]\left[\begin{array}{l}
\underline{y}_{p}(t) \\
\underline{y}_{q}(t)
\end{array}\right]+\left[\begin{array}{l}
\tilde{\tilde{b}}_{p} \\
\tilde{\tilde{b}}_{q}
\end{array}\right] u_{o}(t),
\end{gathered}
$$

we arrive easily at (23) and (24).

System (23) is standard form of nonhomogeneous higherorder linear differential equations of Apostol-Kolodner type, which may be treated by methods that are more classical; see for instance [18] and references therein.

Thus, it is convenient to define new variables as

$$
\begin{gathered}
\underline{z}_{1}(t)=\underline{y}_{p}(t), \\
\underline{z}_{2}(t)=\underline{y}_{p}^{\prime}(t), \\
\vdots \\
\underline{z}_{r}(t)=\underline{y}_{p}^{(r-1)}(t) .
\end{gathered}
$$

Then, we have the following system of ordinary differential equations:

$$
\begin{gathered}
\underline{z}_{1}^{\prime}(t)=\underline{z}_{2}(t), \\
\underline{z}_{2}^{\prime}(t)=\underline{z}_{3}(t), \\
\vdots \\
\underline{z}_{r}^{\prime}(t)=J_{p} \underline{z}_{1}(t)+\underline{\tilde{b}}_{p} u_{o}(t) .
\end{gathered}
$$

Finally, (31) can be expressed by using vector-matrix equations

$$
\underline{\mathbf{z}}^{\prime}(t)=\mathbf{R} \underline{\mathbf{z}}(t)+\underline{\mathbf{L}} u_{o}(t),
$$

where $\underline{\mathbf{z}}(t)=\left[\begin{array}{llll}\underline{z}_{1}^{t}(t) & \underline{z}_{2}^{t}(t) & \cdots & \underline{z}_{r}^{t}(t)\end{array}\right]^{t}\left(()^{t}\right.$ is the transpose tensor) and the coefficient matrices $\mathbf{R}$ and $\underline{\mathbf{L}}$ are given by

$$
\mathbf{R}=\left[\begin{array}{ccccc}
\mathbb{O}_{p} & I_{p} & \mathbb{O}_{p} & \cdots & \mathbb{O}_{p} \\
\mathbb{O}_{p} & \mathbb{O}_{p} & I_{p} & \cdots & \mathbb{O}_{p} \\
\vdots & \vdots & \vdots & \ddots & \vdots \\
\mathbb{O}_{p} & \mathbb{O}_{p} & \mathbb{O}_{p} & \cdots & I_{p} \\
J_{p} & \mathbb{O}_{p} & \mathbb{O}_{p} & \cdots & \mathbb{O}_{p}
\end{array}\right] \in \mathcal{M}_{r p}
$$

$$
\underline{\mathbf{L}}=\left[\begin{array}{c}
\underline{0}_{p} \\
\underline{0}_{p} \\
\vdots \\
\tilde{b}_{p}
\end{array}\right] \in \mathcal{M}_{(r p) 1} \text {, }
$$

with corresponding dimension of $\underline{\mathbf{z}}(t), r p \times 1$.

The state of system $(1)$ at time $0^{-}$is $\underline{x}\left(0^{-}\right)=\left[\begin{array}{llll}0 & 0 & \cdots & 0\end{array}\right]^{t}$ and at time $0^{+}$it achieves $\underline{x}\left(0^{+}\right)=\left[\begin{array}{llll}x_{1} & x_{2} & \cdots & x_{n}\end{array}\right]^{t}$.

Now, considering (25), at time $0^{-}$we have

$$
\begin{aligned}
\underline{y}\left(0^{-}\right) & =Q^{-1} \underline{x}\left(0^{-}\right)=\underline{y}^{\prime}\left(0^{-}\right) \\
& =Q^{-1} \underline{x}^{\prime}\left(0^{-}\right)=\cdots=\underline{y}^{(r-1)}\left(0^{-}\right) \\
& =Q^{-1} \underline{x}^{(r-1)}\left(0^{-}\right)=\underline{0}^{t},
\end{aligned}
$$

and at time $0^{+}$we obtain

$$
\begin{gathered}
\underline{y}\left(0^{+}\right)=Q^{-1} \underline{x}\left(0^{+}\right)=\left[\begin{array}{llll}
\chi_{1}^{0} & \chi_{2}^{0} & \cdots & \chi_{n}^{0}
\end{array}\right]^{t}, \\
\underline{y^{\prime}}\left(0^{+}\right)=Q^{-1} \underline{x}^{\prime}\left(0^{+}\right)=\left[\begin{array}{llll}
\chi_{1}^{1} & \chi_{2}^{1} & \cdots & \chi_{n}^{1}
\end{array}\right]^{t}, \ldots, \\
\underline{y}^{(r-1)}\left(0^{+}\right)=Q^{-1} \underline{x}^{(r-1)}\left(0^{+}\right)=\left[\begin{array}{llll}
\chi_{1}^{r-1} & \chi_{2}^{r-1} & \cdots & \chi_{n}^{r-1}
\end{array}\right]^{t} .
\end{gathered}
$$

Moreover,

$$
\begin{gathered}
\underline{y}_{p}^{(i)}\left(0^{-}\right)=\underline{0}_{p}^{t} \in \mathcal{M}_{p}, \\
\underline{y}_{p}^{(i)}\left(0^{+}\right)=\left[\begin{array}{llll}
\chi_{1}^{i} & \chi_{2}^{i} & \cdots & \chi_{p}^{i}
\end{array}\right]^{t} \in \mathcal{M}_{p}, \\
\underline{y}_{q}^{(i)}\left(0^{-}\right)=\underline{0}_{q}^{t} \in \mathcal{M}_{q}, \\
\underline{y}_{q}^{(i)}\left(0^{+}\right)=\left[\begin{array}{llll}
\chi_{p+1}^{i} & \chi_{p+2}^{i} & \cdots & \chi_{n}^{i}
\end{array}\right]^{t} \in \mathcal{M}_{q},
\end{gathered}
$$

where $\chi_{j}^{i} \in \mathbb{F}$ for $i=0,1,2, \ldots, r-1$, and $j=1,2, \ldots, n$. 
Furthermore,

$$
\begin{aligned}
& \underline{\mathbf{z}}\left(0^{-}\right)=\left[\begin{array}{llll}
\underline{z}_{1}^{t}\left(0^{-}\right) & \underline{z}_{2}^{t}\left(0^{-}\right) & \cdots & \underline{z}_{r}^{t}\left(0^{-}\right)
\end{array}\right]^{t} \\
& =\left[\begin{array}{llll}
\underline{y}_{p}^{t}\left(0^{-}\right) & \underline{y}_{p}^{\prime t}\left(0^{-}\right) & \cdots & \underline{y}_{p}^{(r-1) t}\left(0^{-}\right)
\end{array}\right]^{t}=\underline{0}_{r p}^{t}, \\
& \underline{\mathbf{z}}\left(0^{+}\right)=\left[\begin{array}{llll}
\underline{z}_{1}^{t}\left(0^{+}\right) & \underline{z}_{2}^{t}\left(0^{+}\right) & \cdots & \underline{z}_{r}^{t}\left(0^{+}\right)
\end{array}\right]^{t} \\
& =\left[\begin{array}{llll}
\underline{y}_{p}^{t}\left(0^{+}\right) & \underline{y}_{p}^{\prime t}\left(0^{+}\right) & \cdots & \underline{y}_{p}^{(r-1) t}\left(0^{+}\right)
\end{array}\right]^{t} \\
& =\left[\begin{array}{lllllll}
\chi_{1}^{0} & \chi_{2}^{0} & \cdots & \chi_{p}^{0} & \chi_{1}^{1} & \chi_{2}^{1} & \cdots
\end{array}\right. \\
& \left.\begin{array}{llllll}
\chi_{p}^{1} & \cdots & \chi_{1}^{r-1} & \chi_{2}^{r-1} & \cdots & \chi_{p}^{r-1}
\end{array}\right]^{t} .
\end{aligned}
$$

3.2. The Solution of Subsystem (32). In order to solve subsystem (32), the following definitions should be provided.

Definition 4. The characteristic polynomial of matrix $\mathbf{R}$ is given by

$$
\varphi(\lambda)=\prod_{i=1}^{\kappa}\left(\lambda-\lambda_{1}\right)^{\tau_{i}}
$$

with $\lambda_{i} \neq \lambda_{j}$ for $i \neq j$ and $\sum_{i=1}^{\kappa} \tau_{i}=r p$. Without loss of generality, we assume that

$$
\begin{aligned}
& d_{1}=\tau_{1}, \quad d_{2}=\tau_{2}, \ldots, \\
& d_{l}=\tau_{l}, \quad \text { for } 1<l<\kappa, d_{l+1}<\tau_{l+1}, \ldots, d_{k}<\tau_{\kappa},
\end{aligned}
$$

where $d_{i}, \tau_{i}, i=1,2, \ldots, \kappa$ is the geometric and algebraic multiplicity of the given eigenvalues $\lambda_{i}$, respectively.

Generally speaking, the matrix $\mathbf{R}$ is not diagonalizable. However, we can generate $r p$ linearly independent vectors $\underline{v}_{1}, \underline{v}_{2}, \ldots, \underline{v}_{r p}$ and $r p \times r p$ similarity transformation $C=$ $\left[\underline{v}_{1}, \underline{v}_{2}, \ldots, \underline{v}_{r p}\right]$ that takes $\mathbf{R}$ into the Jordan canonical form, as the following definition clarifies.

Definition 5. There exists an invertible matrix $C \in \mathcal{M}_{r p}$ such as $\mathbf{J}=C^{-1} \mathbf{R} C, \mathbf{J} \in \mathcal{M}_{r p}$; is it Jordan canonical form of matrix R. Analytically,

$$
\mathbf{J}=J_{o} \bigoplus J_{l+1} \bigoplus J_{l+2} \bigoplus \cdots \oplus J_{\kappa} .
$$

(i) The block diagonal matrix $J_{o}=J_{1} \oplus J_{2} \oplus \cdots \oplus J_{l}$, where

$$
J_{i}=\left[\begin{array}{llll}
\lambda_{i} & & & \\
& \lambda_{i} & & \\
& & \ddots & \\
& & & \lambda_{i}
\end{array}\right] \in \mathcal{M}_{\tau_{i}},
$$

is also a diagonal matrix with diagonal elements the eigenvalue $\lambda_{i}$, for $i=1, \ldots, l$. Consequently, the dimension of $J_{o}$ is $s \times s, s \triangleq \sum_{i=1}^{l} \tau_{i}$. (ii) Also, the block matrix $J_{j}=J_{j, 1} \oplus J_{j, 2} \oplus \cdots \oplus J_{j, d_{j}} \in$ $\mathcal{M}_{\tau_{j}}$, where

$$
J_{j, z_{j}}=\left[\begin{array}{ccccc}
\lambda_{j} & 1 & & & \\
& \lambda_{j} & 1 & & \\
& & \lambda_{j} & \ddots & \\
& & & \ddots & 1 \\
& & & & \lambda_{j}
\end{array}\right] \in \mathcal{M}_{z_{j}}
$$

$$
\text { for } j=l+1, l+2, \ldots, k, z_{j}=1,2, \ldots, d_{j} \text {. }
$$

According to the classical theory of ordinary differential equations, the solution of system (32) is given by the following lemma.

Lemma 6. The solution of subsystem (32) is given by

$$
\underline{\mathbf{z}}(t)=C \int_{-\infty}^{t} e^{\mathrm{J}(t-\tau)} \underline{\mathbf{b}} u_{o}(\tau) d \tau
$$

with initial condition $\underline{\mathbf{z}}\left(0^{-}\right)=\underline{0}_{r p}^{t}$.

Proof. Consider the transformation

$$
\underline{\mathbf{z}}(t)=C \underline{\zeta}(t),
$$

where nonsingular $C \in \mathcal{M}_{r p}$, and $\underline{\zeta}\left(0^{-}\right)=C^{-1} \underline{\mathbf{z}}\left(0^{-}\right)=\underline{0}_{r p}^{t}$.

Substituting (44) into (43), we obtain

$$
\begin{aligned}
C \underline{\zeta}^{\prime}(t) & =\mathbf{R} C \underline{\zeta}(t)+\underline{\mathbf{L}} u_{o}(t) \Longleftrightarrow \underline{\zeta}^{\prime}(t) \\
& =C^{-1} \mathbf{R} C \underline{\zeta}(t)+C^{-1} \underline{\mathbf{L}} u_{o}(t) .
\end{aligned}
$$

Furthermore, we define $\underline{\mathbf{b}} \triangleq C^{-1} \underline{\mathbf{L}}$, such as the last equation is transformed into

$$
\underline{\zeta}^{\prime}(t)=\mathbf{J} \underline{\zeta}(t)+\underline{\mathbf{b}} u_{o}(t) .
$$

Now, according to the relevant theory of first-order differential systems of (46) form, see for instant [16], using also (44), the solution is expressed by (43).

Definition 7. The exponential matrix $e^{J(t-\tau)}$ is defined as

$$
\begin{aligned}
& e^{J(t-\tau)} \\
& =e^{J_{\sigma}(t-\tau)} \bigoplus e^{J_{l+1}(t-\tau)} \bigoplus e^{J_{++2}(t-\tau)} \bigoplus \cdots \bigoplus e^{J_{\kappa}(t-\tau)} \in \mathcal{M}_{r p},
\end{aligned}
$$

where

$$
\begin{aligned}
& e^{J_{o}(t-\tau)}=e^{J_{1}(t-\tau)} \bigoplus e^{J_{2}(t-\tau)} \bigoplus \cdots \bigoplus e^{J_{l}(t-\tau)} \in \mathcal{M}_{s}, \\
& e^{J_{i}(t-\tau)}=e^{\lambda_{i}(t-\tau)} \bigoplus e^{\lambda_{i}(t-\tau)} \bigoplus \cdots \bigoplus e^{\lambda_{i}(t-\tau)} \in \mathcal{M}_{\tau_{i}}, \\
& \text { for } i=1, \ldots, l . \\
& \quad \text { Furthermore, }
\end{aligned}
$$

$$
e^{J_{j}(t-\tau)}=e^{\lambda_{j_{1}}(t-\tau)} \bigoplus e^{\lambda_{j_{2}}(t-\tau)} \bigoplus \cdots \bigoplus e^{\lambda_{j_{j}}(t-\tau)} \in \mathcal{M}_{\tau_{j}},
$$


where

$$
e^{J_{j, z_{j}}(t-\tau)}=e^{\lambda_{j}(t-\tau)}\left[\begin{array}{cccc}
1 & t-\tau & \cdots & \frac{(t-\tau)^{\mu_{z_{j}}-1}}{\left(\mu_{z_{j}}-1\right) !} \\
0 & 1 & \cdots & \frac{(t-\tau)^{\mu_{z_{j}}-2}}{\left(\mu_{z_{j}}-2\right) !} \\
\vdots & \vdots & \ddots & \vdots \\
0 & 0 & \cdots & 1
\end{array}\right] \in \mathcal{M}_{\mu_{z_{j}}},
$$

where $\mu_{z_{j}}$ are the Weyr characteristics via Ferrer diagrams, for $j=l+1, l+2, \ldots, k$ and $z_{j}=1,2, \ldots, d_{j}$. Note that $\rho_{j}=\max _{z_{i}=1,2, \ldots, d_{j}} \mu_{z_{j}}$ is the index of annihilation for the eigenvalue $\lambda_{j}$.

Now, taking into consideration (21) the solution (22) is transposed into

$$
\underline{\mathbf{z}}(t)=\lim _{\sigma \rightarrow 0}\left\{C e^{\mathrm{Jt}} \int_{-\infty}^{t} e^{-\mathbf{J} \tau} \underline{\mathbf{b}} u(\tau) d \tau\right\},
$$

or equivalently

$$
\underline{\mathbf{z}}(t)=C\left[\begin{array}{c}
\lim _{\sigma \rightarrow 0}\left\{e^{J_{o} t} \int_{-\infty}^{t} e^{-J_{o} \tau} \underline{\mathbf{b}}_{s} u(\tau) d \tau\right\} \\
\lim _{\sigma \rightarrow 0}\left\{e^{J_{l+1} t} \int_{-\infty}^{t} e^{-J_{l+1} \tau} \underline{\mathbf{b}}_{\tau_{l+1}} u(\tau) d \tau\right\} \\
\vdots \\
\lim _{\sigma \rightarrow 0}\left\{e^{J_{k} t} \int_{-\infty}^{t} e^{-J_{\kappa} \tau} \underline{\mathbf{b}}_{\tau_{\kappa}} u(\tau) d \tau\right\}
\end{array}\right],
$$

where $\underline{\mathbf{b}}=\left[\begin{array}{lll}\underline{\mathbf{b}}_{s}^{t} \underline{\mathbf{b}}_{\tau_{l+1}}^{t} & \cdots & \underline{\mathbf{b}}_{\tau_{\kappa}}^{t}\end{array}\right]^{t}$.

Remark 1. [4, 5] Given the large number of terms involved, in order to make our calculations affordable, we consider the fact that $\Phi(t / \sigma)$ and its derivatives tend to zero very strongly with $t \rightarrow 0$ (note also that $\sigma \rightarrow 0$ ). Thus, by letting $t=$ $K \cdot \sigma$, where $K$ is chosen large enough (i.e., $K \rightarrow \infty$ ) the assumption as stated above is valid, that is,

$$
\Phi\left(\frac{t}{\sigma}\right) \stackrel{t=K \sigma}{=} \Phi(K) \longrightarrow 0,
$$

and its derivatives $\Phi^{(i)}(t / \sigma) \stackrel{t=K \sigma}{=} \Phi^{(i)}(K) \stackrel{K \rightarrow \infty}{\rightarrow} 0$, for $i=$ $0,1,2, \ldots, k-1$.

Now, using the Remark 1, we obtain that $\lim _{\sigma \rightarrow 0} e^{J_{i} t} \stackrel{t=K \sigma}{=} I$, for $i=1,2, \ldots, l, l+1, l+2, \ldots, k$, and $\Phi^{-1}\left(K+\lambda_{i} \sigma\right) \rightarrow$ $\Phi^{-1}(K)$, as well. Moreover, for the analytic determination of vector (3), we need to calculate the integral $\int_{-\infty}^{t} e^{-J \tau} \underline{\mathbf{b}} u(\tau) d \tau$.

In this part of the paper, two subsystems are derived; see the following lemmas, and Appendices A and B.

Lemma 8. For the diagonal matrix $J_{o}$, the following integral is given by (54):

$$
\lim _{\sigma \rightarrow 0} \int_{-\infty}^{t} e^{-J_{0} t} \underline{\mathbf{b}}_{s} u(\tau) d \tau=\Phi^{-1}(K)\left[\begin{array}{c}
\underline{\mathbf{b}}_{\tau_{1}} \sum_{k=0}^{n-1} \alpha_{k} \lambda_{1}^{k} \\
\vdots \\
\underline{\mathbf{b}}_{\tau_{i}} \sum_{k=0}^{n-1} \alpha_{k} \lambda_{i}^{k} \\
\vdots \\
\underline{\mathbf{b}}_{\tau_{l}} \sum_{k=0}^{n-1} \alpha_{k} \lambda_{l}^{k}
\end{array}\right] \in \mathcal{M}_{s 1} .
$$

Proof. See Appendix A.

Lemma 9. For the diagonal matrix $J_{j}=J_{j_{1}} \oplus J_{j_{2}} \oplus \cdots \oplus J_{j_{d_{j}}}$ $\in \mathcal{M}_{\tau_{j}}$, the following integral is given by (55):

$$
\lim _{\sigma \rightarrow 0} \int_{-\infty}^{t} e^{-J_{j} t} \underline{\mathbf{b}}_{\tau_{j}} u(\tau) d \tau=\Phi^{-1}(K)\left[\begin{array}{c}
\mathbf{V}_{j_{1}} \\
\hline \mathbf{V}_{j_{2}} \\
\hline \vdots \\
\hline \mathbf{V}_{j_{j}} \\
\hline \vdots \\
\hline \mathbf{V}_{j_{j}} \\
\hline
\end{array}\right]
$$

where

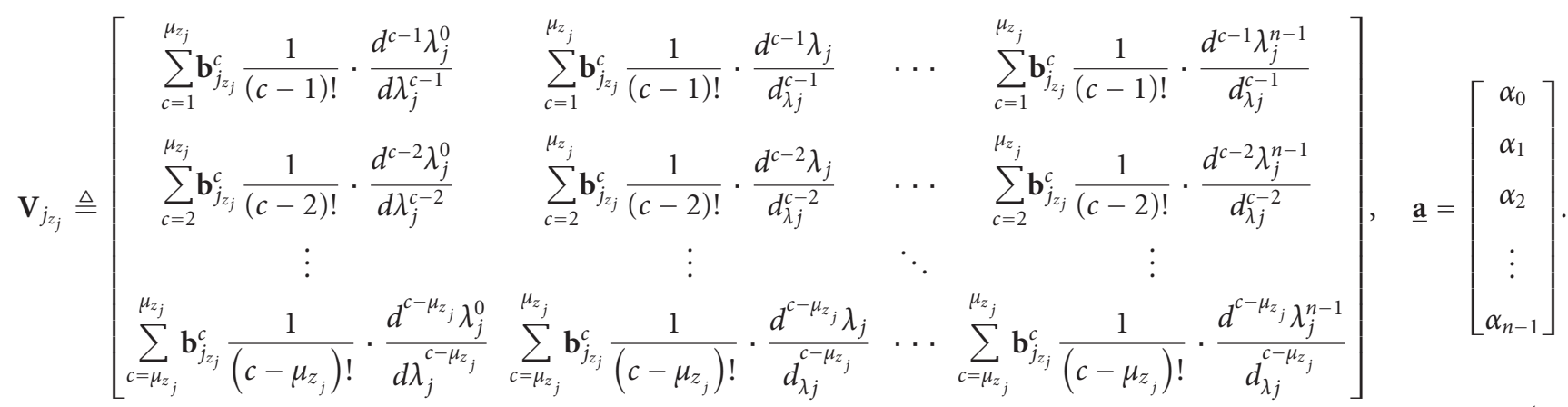


Proof. See Appendix B.

Now, we revisit (54), thus

$$
\Phi^{-1}(K)\left[\begin{array}{c}
\underline{\mathbf{b}}_{\tau_{1}} \sum_{k=0}^{n-1} \alpha_{k} \lambda_{1}^{k} \\
\vdots \\
\underline{\mathbf{b}}_{\tau_{i}} \sum_{k=0}^{n-1} \alpha_{k} \lambda_{i}^{k} \\
\vdots \\
\underline{\mathbf{b}}_{\tau_{l}} \sum_{k=0}^{n-1} \alpha_{k} \lambda_{l}^{k}
\end{array}\right]
$$$$
=\Phi^{-1}(K)\left(\underline{\mathbf{b}}_{\tau_{1}} \oplus \underline{\mathbf{b}}_{\tau_{2}} \oplus \cdots \oplus \underline{\mathbf{b}}_{\tau_{l}}\right)\left[\begin{array}{c}
\sum_{k=0}^{n-1} \alpha_{k} \lambda_{1}^{k} \\
\sum_{k=0}^{n-1} \alpha_{k} \lambda_{2}^{k} \\
\vdots \\
\sum_{k=0}^{n-1} \alpha_{k} \lambda_{l}^{k}
\end{array}\right]
$$$$
=\Phi^{-1}(K)\left(\underline{\mathbf{b}}_{\tau_{1}} \bigoplus \underline{\mathbf{b}}_{\tau_{2}} \bigoplus \cdots \oplus \underline{\mathbf{b}}_{\tau_{l}}\right)
$$$$
\times \underbrace{\left[\begin{array}{ccccc}
1 & \lambda_{1} & \lambda_{1}^{2} & \cdots & \lambda_{1}^{n-1} \\
1 & \lambda_{2} & \lambda_{2}^{2} & \cdots & \lambda_{2}^{n-1} \\
\vdots & \vdots & \vdots & \ddots & \vdots \\
1 & \lambda_{l} & \lambda_{l}^{2} & \cdots & \lambda_{l}^{n-1}
\end{array}\right]}_{\mathbf{V}_{l}}\left[\begin{array}{c}
\alpha_{0} \\
\alpha_{1} \\
\alpha_{2} \\
\vdots \\
\alpha_{n-1}
\end{array}\right]
$$$$
=\Phi^{-1}(K)\left(\underline{\mathbf{b}}_{\tau_{1}} \bigoplus \underline{\mathbf{b}}_{\tau_{2}} \bigoplus \cdots \bigoplus \underline{\mathbf{b}}_{\tau_{l}}\right) \mathbf{V}_{l} \underline{\mathbf{a}} .
$$

Note that $\mathbf{V}_{l}$ is the well-known Vandermonde matrix.

Combining (52)-(57), we obtain the following system:

$$
\begin{aligned}
C^{-1} \underline{\mathbf{z}}\left(0^{+}\right) \\
=\Phi^{-1}(K) \\
\quad \times(\underbrace{\left.\underline{\mathbf{b}}_{\tau_{1}} \oplus \underline{\mathbf{b}}_{\tau_{2}} \oplus \cdots \oplus \underline{\mathbf{b}}_{\tau_{l}} \oplus I_{\tau_{l+1}} \oplus \cdots \oplus I_{\tau_{\kappa}}\right)}_{l}
\end{aligned}
$$

$$
\times\left[\begin{array}{c}
\mathbf{V}_{l} \\
\mathbf{V}_{(l+1)_{1}} \\
\vdots \\
\mathbf{V}_{(l+1)_{d_{l+1}}} \\
\vdots \\
\mathbf{V}_{j_{1}} \\
\vdots \\
\mathbf{V}_{j_{d_{j}}} \\
\vdots \\
\mathbf{V}_{\kappa_{1}} \\
\vdots \\
\mathbf{V}_{\kappa_{d_{k}}}
\end{array}\right]
$$

where $\mathbf{V}_{l} \in \mathcal{M}_{s n}$ and $\mathbf{V}_{j_{z_{j}}} \in \mathcal{M}_{\mu_{z_{j}} n}$ with $j=l+1, l+2, \ldots, \kappa$ and $z_{j}=1,2, \ldots, d_{j}$. Furthermore, we obtain the initial condition

$$
\begin{aligned}
C^{-1} \underline{\mathbf{z}}\left(0^{+}\right) \\
=\left[\begin{array}{lllllll}
\underline{\mathbf{z}}_{l}^{t}\left(0^{+}\right) & \underline{\mathbf{z}}_{(l+1)_{1}}^{t}\left(0^{+}\right) & \cdots & \underline{\mathbf{z}}_{(l+1)_{d_{l+1}}^{t}}^{t}\left(0^{+}\right) & \cdots & \underline{\mathbf{z}}_{j_{1}}^{t}\left(0^{+}\right) \\
& \cdots & \underline{\mathbf{z}}_{j_{d_{j}}}^{t}\left(0^{+}\right) & \cdots & \underline{\mathbf{z}}_{\kappa_{1}}^{t}\left(0^{+}\right) & \cdots & \underline{\mathbf{z}}_{\kappa_{d_{k}}}^{t}\left(0^{+}\right)
\end{array}\right]
\end{aligned}
$$

The system (58) can now be divided into two types of subsystems:

(S1)

$$
\frac{1}{\Phi^{-1}(K)} \underline{\mathbf{z}}_{l}\left(0^{+}\right)=\left(\underline{\mathbf{b}}_{\tau_{1}} \bigoplus \underline{\mathbf{b}}_{\tau_{2}} \bigoplus \cdots \bigoplus \underline{\mathbf{b}}_{\tau_{l}}\right) \mathbf{V}_{l} \underline{\mathbf{a}},
$$

$$
\frac{1}{\Phi^{-1}(K)^{2}} \underline{\mathbf{z}}_{j_{j}}\left(0^{+}\right)=\mathbf{V}_{j_{z_{j}}} \mathbf{a},
$$

for $j=l+1, l+2, \ldots, \kappa$ and $z_{j}=1,2, \ldots, d_{j}$.

Proposition 10. System (60) is solvable if

$$
\frac{z_{i}^{1}}{b_{i}^{1}}=\frac{z_{i}^{2}}{b_{i}^{2}}=\cdots=\frac{z_{i}^{\tau_{i}}}{b_{i}^{\tau_{i}}},
$$

for every nonzero element of vectors $\underline{\mathbf{b}}_{\tau_{1}}, \underline{\mathbf{b}}_{\tau_{2}}, \ldots, \underline{\mathbf{b}}_{\tau_{l}}$, where $\underline{z}_{i}\left(0^{+}\right)=\left[\begin{array}{llll}z_{i}^{1} & z_{i}^{2} & z_{i}^{\tau_{i}}\end{array}\right]^{t}$, for every $i=1,2, \ldots, l$.

Moreover, if one of the elements of vectors $\underline{\mathbf{b}}_{\tau_{1}}, \underline{\mathbf{b}}_{\tau_{2}}, \ldots, \underline{\mathbf{b}}_{\tau_{l}}$ is zero, then the relative element of the ith-row of the vector $\underline{\mathbf{z}}_{\tau_{i}}\left(0^{+}\right)$should be also zero. 
Proof. System (60) contains $l$-subsystems of the following type:

$$
\begin{aligned}
\left(\underline{0}_{\tau_{1}} \bigoplus \cdots \bigoplus \underline{\mathbf{b}}_{\tau_{i}} \bigoplus \cdots \bigoplus \underline{0}_{\tau_{l}}\right) \mathbf{v}_{l} \underline{\boldsymbol{\alpha}} \\
\quad=\frac{1}{\Phi^{-1}(K)}\left(I_{\tau_{1}} \bigoplus \cdots \bigoplus I_{\tau_{i}} \bigoplus \cdots \bigoplus I_{\tau_{l}}\right) \underline{\mathbf{z}}_{l}\left(0^{+}\right),
\end{aligned}
$$

or equivalently

$$
\left[\begin{array}{cccc}
b_{i}^{1} & b_{i}^{1} \lambda_{i} & \cdots & b_{i}^{1} \lambda_{i}^{n-1} \\
b_{i}^{2} & b_{i}^{2} \lambda_{i} & \cdots & b_{i}^{2} \lambda_{i}^{n-1} \\
\vdots & \vdots & \ddots & \vdots \\
b_{i}^{\tau_{i}} & b_{i}^{\tau_{i}} \lambda_{i} & \cdots & b_{i}^{\tau_{i}} \lambda_{i}^{n-1}
\end{array}\right]\left[\begin{array}{c}
\alpha_{0} \\
\alpha_{1} \\
\vdots \\
\alpha_{n-1}
\end{array}\right]=\frac{1}{\Phi^{-1}(K)}\left[\begin{array}{c}
z_{i}^{1}\left(0^{+}\right) \\
z_{i}^{2}\left(0^{+}\right) \\
\vdots \\
z_{i}^{\tau_{i}}\left(0^{+}\right)
\end{array}\right] .
$$
$\tau_{i}$, then the relative element of the $i$ th row of the vector $\underline{\mathbf{z}}_{\tau_{i}}\left(0^{+}\right)$should be also zero (in order to obtain solution).

(ii) If every of the coefficients $b_{i}^{\gamma} \neq 0$, for $\gamma=1,2, \ldots, \tau_{i}$, then we have (i) If one of the coefficients $b_{i}^{\gamma}=0$, for $\gamma=1,2, \ldots$,

$$
\left.\begin{array}{c}
b_{i}^{1}\left(\alpha_{0}+\alpha_{1} \lambda_{i}+\cdots+\alpha_{n-1} \lambda_{i}^{n-1}\right)=z_{i}^{1} \frac{1}{\Phi^{-1}(K)} \\
b_{i}^{2}\left(\alpha_{0}+\alpha_{1} \lambda_{i}+\cdots+\alpha_{n-1} \lambda_{i}^{n-1}\right)=z_{i}^{2} \frac{1}{\Phi^{-1}(K)} \\
\vdots \\
b_{i}^{\tau_{i}}\left(\alpha_{0}+\alpha_{1} \lambda_{i}+\cdots+\alpha_{n-1} \lambda_{i}^{n-1}\right)=z_{i}^{\tau_{i}} \frac{1}{\Phi^{-1}(K)}
\end{array}\right\} \Leftrightarrow
$$

for every $i=1,2, \ldots, l$.

Consequently, system (60) is solvable if (62) is satisfied for every $i=1,2, \ldots, l$.

Now, we will work with subsystems (61) which can be written as follows:

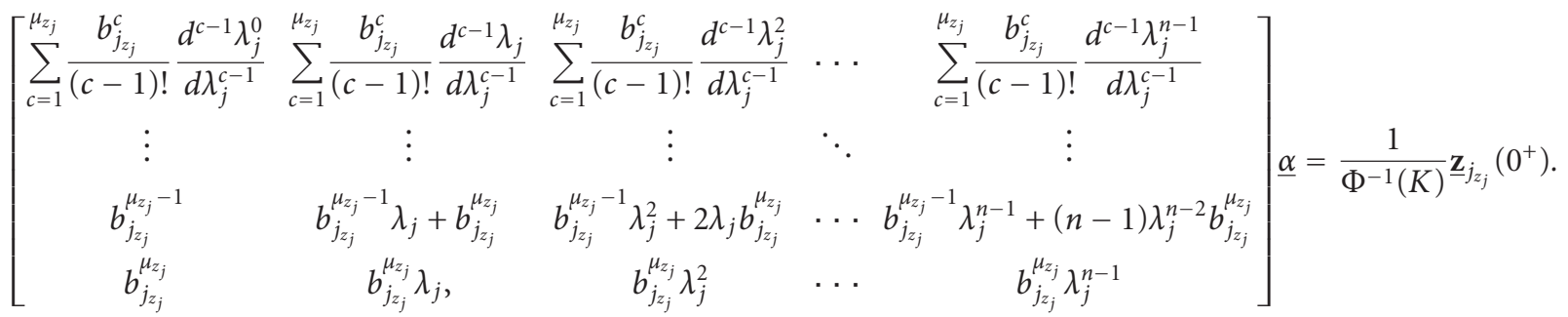

The coefficient matrix $\mathbf{V}_{j_{z j}}$ can be transposed to the following equivalent matrix; see (67). Note that $\mathbf{V}_{j_{z_{j}}}$ is a $\mu_{z_{j}} \times n$ matrix. Moreover, from the $\mu_{z_{j}}$ elements $b_{j_{z_{j}}}^{c}$, for $c=1,2, \ldots, \mu_{z_{j}}$, we can assume that $b_{j_{z_{j}}}^{\mu_{z_{j}}}=b_{j_{z_{j}}}^{\mu_{z_{j}}-1}=\cdots=$ $b_{j_{z_{j}}}^{\mu_{z_{j}}-\mu}=0$. Thus, we obtain

$$
\mathbf{V}_{j_{j}}^{*}=\left[\begin{array}{cccccccc}
0 & 0 & 0 & \cdots & 1 & \frac{1}{\left(\mu_{z_{j}}-\mu\right) !} \frac{d^{\mu_{z_{j}}-\mu} \lambda_{j}^{\mu_{z_{j}}+1-\mu}}{d \lambda_{j}^{\mu_{z_{j}}-\mu}} & \cdots & \frac{1}{\left(\mu_{z_{j}}-\mu\right) !} \frac{d^{\mu_{z_{j}}-\mu} \lambda_{j}^{n-1}}{d \lambda_{j}^{\mu_{z_{j}}-\mu}} \\
\vdots & \vdots & \vdots & \cdots & \vdots & \ddots & \vdots \\
0 & 0 & 1 & \cdots & & \cdots & \frac{1}{2 !} \frac{d^{2} \lambda_{j}^{n-1}}{d \lambda_{j}^{2}} \\
0 & 1 & 2 \lambda_{j} & \cdots & & \cdots & (n-1) \lambda_{j}^{n-1} \\
1 & \lambda_{j} & \lambda_{j}^{2} & \cdots & & \cdots & \lambda_{j}^{n-1} \\
0 & 0 & 0 & \cdots & 0 & 0 & \vdots & 0 \\
\vdots & \vdots & \vdots & \ddots & \vdots & 0 & \cdots & \vdots \\
0 & 0 & 0 & \cdots & 0 & \vdots & \cdots & 0
\end{array}\right] .
$$


In order to be able to understand (67) better, the following example is helpful.

Example 1. We take the coefficient matrix $\mathbf{V}$

$$
\left[\begin{array}{ccccc}
b_{3} & b_{3} \lambda+b_{4} & b_{3} \lambda^{2}+2 \lambda b_{4}+b_{5} & b_{3} \lambda^{3}+3 \lambda^{2} b_{4}+3 \lambda b_{5}+b_{6} & b_{3} \lambda^{4}+4 \lambda^{3} b_{4}+6 \lambda^{2} b_{5}+2 \lambda b_{6} \\
b_{4} & b_{4} \lambda+b_{5} & b_{4} \lambda^{2}+2 \lambda b_{5}+b_{6} & b_{4} \lambda^{3}+3 \lambda^{2} b_{5}+3 \lambda b_{6} & b_{4} \lambda^{4}+4 \lambda^{3} b_{5}+6 \lambda^{2} b_{6} \\
b_{5} & b_{5} \lambda+b_{6} & b_{5} \lambda^{2}+2 \lambda b_{6} & b_{5} \lambda^{3}+3 \lambda^{2} b_{6} & b_{5} \lambda^{4}+4 \lambda^{3} b_{6} \\
b_{6} & b_{6} \lambda & b_{6} \lambda^{2} & b_{6} \lambda^{3} & b_{6} \lambda^{4}
\end{array}\right]
$$

We assume that $b_{6}=0$ and $b_{5} \neq 0$. Since $b_{5} \neq 0$, we are not interested in $b_{3}, b_{4}$.

Thus, under our assumption, we obtain

$$
\left[\begin{array}{ccccc}
b_{3} & b_{3} \lambda+b_{4} & b_{3} \lambda^{2}+2 \lambda b_{4}+b_{5} & b_{3} \lambda^{3}+3 \lambda^{2} b_{4}+3 \lambda b_{5} & b_{3} \lambda^{4}+4 \lambda^{3} b_{4}+6 \lambda^{2} b_{5} \\
b_{4} & b_{4} \lambda+b_{5} & b_{4} \lambda^{2}+2 \lambda b_{5} & b_{4} \lambda^{3}+3 \lambda^{2} b_{5} & b_{4} \lambda^{4}+4 \lambda^{3} b_{5} \\
b_{5} & b_{5} \lambda & b_{5} \lambda^{2} & b_{5} \lambda^{3} & b_{5} \lambda^{4} \\
0 & 0 & 0 & 0 & 0
\end{array}\right] .
$$

Afterwards, we multiply the 3rd row with $-b_{4} / b_{5}$ and it is added to the 2nd row. Moreover, we multiply the 3rd row with $-b_{3} / b_{5}$ and it is added with the 1st row. Then, the following equivalent matrix is derived:

$$
\left[\begin{array}{ccccc}
0 & b_{4} & 2 \lambda b_{4}+b_{5} & 3 \lambda^{2} b_{4}+3 \lambda b_{5} & 4 \lambda^{3} b_{4}+6 \lambda^{2} b_{5} \\
0 & b_{5} & 2 \lambda b_{5} & 3 \lambda^{2} b_{5} & 4 \lambda^{3} b_{5} \\
b_{5} & b_{5} \lambda & b_{5} \lambda^{2} & b_{5} \lambda^{3} & b_{5} \lambda^{4} \\
0 & 0 & 0 & 0 & 0
\end{array}\right]
$$

Now, we multiply the 2 nd row with $-b_{4} / b_{5}$ and it is added with the 1st row. Thus, we obtain

$$
\left[\begin{array}{ccccc}
0 & 0 & b_{5} & 3 \lambda b_{5} & 6 \lambda^{2} b_{5} \\
0 & b_{5} & 2 \lambda b_{5} & 3 \lambda^{2} b_{5} & 4 \lambda^{3} b_{5} \\
b_{5} & b_{5} \lambda & b_{5} \lambda^{2} & b_{5} \lambda^{3} & b_{5} \lambda^{4} \\
0 & 0 & 0 & 0 & 0
\end{array}\right]
$$

Finally, we multiply the matrix with the element $1 / b_{5}$ and we conclude to obtain

$$
\begin{aligned}
{\left[\begin{array}{ccccc}
0 & 0 & 1 & 3 \lambda & 6 \lambda^{2} \\
0 & 1 & 2 \lambda & 3 \lambda^{2} & 4 \lambda^{3} \\
1 & \lambda & \lambda^{2} & \lambda^{3} & \lambda^{4} \\
0 & 0 & 0 & 0 & 0
\end{array}\right] } \\
=\left[\begin{array}{ccccc}
\frac{(1)^{\prime \prime}}{2 !} & \frac{(\lambda)^{\prime \prime}}{2 !} & \frac{\left(\lambda^{2}\right)^{\prime \prime}}{2 !} & \frac{\left(\lambda^{3}\right)^{\prime \prime}}{2 !} & \frac{\left(\lambda^{4}\right)^{\prime \prime}}{2 !} \\
\frac{(1)^{\prime}}{1 !} & \frac{(\lambda)^{\prime}}{1 !} & \frac{\left(\lambda^{2}\right)^{\prime}}{1 !} & \frac{\left(\lambda^{3}\right)^{\prime}}{1 !} & \frac{\left(\lambda^{4}\right)^{\prime}}{1 !} \\
1 & \lambda & \lambda^{2} & \lambda^{3} & \lambda^{4} \\
0 & 0 & 0 & 0 & 0
\end{array}\right] .
\end{aligned}
$$

Remark 2. Considering the results already presented, it is clear that there exists a nonsingular matrix $L_{j_{z_{j}}}, \mu_{z_{j}} \times \mu_{z_{j}}$ such that

$$
L_{j z_{j}} \mathbf{V}_{j z_{j}}=\mathbf{V}_{j_{z_{j}}}^{*} .
$$

Thus, system (61) can be transformed into

$$
\frac{1}{\Phi^{-1}(K)} L_{j z_{j}} \underline{\mathbf{z}}_{j_{z_{j}}}\left(0^{+}\right)=L_{j z_{j}} \mathbf{V}_{j_{z_{j}}} \mathbf{a} \Longleftrightarrow \mathbf{V}_{j_{z_{j}}}^{*} \mathbf{a}=\underline{\mathbf{z}}_{z_{j}}^{*}\left(0^{+}\right),
$$

where

$$
\begin{gathered}
\underline{\mathbf{z}}_{j_{j}}^{*}\left(0^{+}\right)=\frac{1}{\Phi^{-1}(K)} L_{j z_{j}} \underline{\mathbf{z}}_{z_{j}}\left(0^{+}\right), \\
\forall j=l+1, l+2, \ldots, \kappa \\
\forall z_{j}=1,2, \ldots, d_{j} .
\end{gathered}
$$

Proposition 11. Subsystems (74) are solvable when the elements of vectors

$$
\underline{\mathbf{z}}_{j_{j}}^{*}\left(0^{+}\right), \quad \text { for every } z_{j}=1,2, \ldots, d_{j}, j=l+1, l+2, \ldots, \kappa
$$

are included into the vector with the greatest dimension, that is, $\rho_{j} \times 1$ where

$$
\rho_{j}=\max \mu_{z_{j}}, \quad z_{j}=1,2, \ldots, d_{j} .
$$

Equivalently, if one assumes that

$$
\underline{\mathbf{z}}_{j_{1}}^{*}\left(0^{+}\right)=\left[\begin{array}{c}
\gamma_{1} \\
\gamma_{2} \\
\vdots \\
\gamma_{\rho_{j}}
\end{array}\right],
$$

then each of the following vectors $\underline{\mathbf{z}}_{j_{2}}^{*}\left(0^{+}\right), \underline{\mathbf{z}}_{j_{3}}^{*}\left(0^{+}\right), \ldots, \underline{\mathbf{z}}_{j_{j_{j}}}^{*}\left(0^{+}\right)$ should be vectors of $\left[\begin{array}{c}\gamma_{1} \\ y_{2} \\ \vdots \\ y_{\xi}\end{array}\right]$ type with $\xi \leq \rho_{j}$. 
Proof. We take the $d_{j}$ subsystems; as follows:

$$
\begin{gathered}
\mathbf{V}_{j_{1}}^{*} \underline{\mathbf{a}}=\underline{\mathbf{z}}_{j_{1}}^{*}\left(0^{+}\right), \\
\cdots \\
\mathbf{V}_{j_{d_{j}}}^{*} \underline{\mathbf{a}}=\underline{\mathbf{z}}_{j_{d_{j}}}^{*}\left(0^{+}\right) .
\end{gathered}
$$

The matrix

$$
\begin{aligned}
& \mathbf{V}_{j_{1}}^{*} \text { has } \mu_{1} \text { rows, } \\
& \mathbf{V}_{j_{2}}^{*} \text { has } \mu_{2} \text { rows, } \\
& \vdots \\
& \mathbf{V}_{j_{d_{j}}}^{*} \text { has } \mu_{d_{j}} \text { rows. }
\end{aligned}
$$

Without loss of generality, we assume that $\mu_{1} \leq \mu_{2} \leq \cdots \leq$ $\mu_{d_{j}}=\rho_{j}$.

Looking carefully at the type of matrices

$$
\mathbf{V}_{j_{1}}^{*}, \mathbf{V}_{j_{2}}^{*}, \ldots, \mathbf{V}_{j_{d_{j}}}^{*},
$$

we can easily verify that the $\mu_{1}$-first rows of them are identically same. Thus, it is necessary the relevant $\mu_{1}$-first rows of $\underline{\mathbf{z}}_{j_{1}}^{*}\left(0^{+}\right), \underline{\mathbf{z}}_{j_{2}}^{*}\left(0^{+}\right), \ldots, \underline{\mathbf{z}}_{j_{d_{j}}}^{*}\left(0^{+}\right)$to be also identically the same. Analogously, the $\mu_{2}$-first rows of $\mathbf{V}_{j_{1}}^{*}, \mathbf{V}_{j_{2}}^{*}, \ldots, \mathbf{V}_{j_{d_{j}}}^{*}$ should be identically the same with the relevant $\mu_{2}$-first rows of $\underline{\mathbf{z}}_{j_{1}}^{*}\left(0^{+}\right), \underline{\mathbf{z}}_{j_{2}}^{*}\left(0^{+}\right), \ldots, \underline{\mathbf{z}}_{j_{d_{j}}}^{*}\left(0^{+}\right)$. And so on until the $\mu_{d_{j}}=\rho_{j}$ row.

$$
\begin{aligned}
& \Phi^{-1}(K)(\underbrace{\underline{\mathbf{b}}_{\tau_{1}} \bigoplus \underline{\mathbf{b}}_{\tau_{2}} \bigoplus \cdots \bigoplus \underline{\mathbf{b}}_{\tau_{l}} \cdots}_{l} \\
& \left.\bigoplus \frac{1}{\Phi^{-1}(K)}\left[I_{\tau_{l+1}} \bigoplus \cdots \bigoplus I_{\tau_{\kappa}}\right]\right)
\end{aligned}
$$

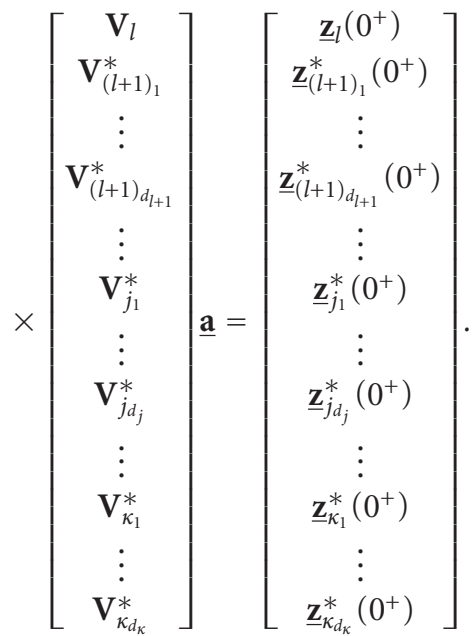

Consequently, it is time to use the results of Propositions 10 and 11. Thus, system (81) is solvable if we obtain the following. (i) The first nonzero elements of vector coefficient $\underline{\mathbf{b}}_{\tau_{1}}, \underline{\mathbf{b}}_{\tau_{2}}, \ldots, \underline{\mathbf{b}}_{\tau_{l}}$ (practically speaking, without loss of generality, we assume that the first elements are nonzero).

(ii) The matrix $\mathbf{V}_{(q+1)_{z_{q+1}}}^{*}$ with dimension $\rho_{l+1} \times n$, where $\rho_{l+1}=\max \mu_{z_{l+1}}, z_{l+1}=1,2, \ldots, d_{l+1}$.

(iii) The matrix $\mathbf{V}_{j_{z_{j}}}^{*}$ with dimension $\rho_{j} \times n$, where $\rho_{j}=$ $\max \mu_{z_{j}}, z_{j}=1,2, \ldots, d_{j}$.

(iv) The matrix $\mathbf{V}_{\kappa_{z_{\kappa}}}^{*}$ with dimension $\rho_{\kappa} \times n$, where $\rho_{\kappa}=$ $\max \mu_{z_{\kappa}}, z_{\kappa}=1,2, \ldots, d_{\kappa}$.

Consequently, system (81) is transposed to the solvable system (83)

$$
\begin{aligned}
& \Phi^{-1}(K)(\underbrace{b_{\tau_{1}} \bigoplus b_{\tau_{2}} \bigoplus \cdots \bigoplus b_{\tau_{l}}}_{l} \\
& \bigoplus \frac{1}{\Phi^{-1}(K)}\left[\begin{array}{c}
\left.\left.I_{\tau_{l+1}} \bigoplus \cdots \bigoplus I_{\tau_{\kappa}}\right]\right) \\
\times
\end{array}\right. \\
& {\left[\begin{array}{c}
\mathbf{V}_{l} \\
\mathbf{V}_{(l+1)_{z_{l+1}}}^{*} \\
\mathbf{V}_{(l+2)_{z_{l+2}}^{*}}^{*} \\
\vdots \\
\mathbf{V}_{\mathcal{K}_{d_{\kappa}}^{*}}^{*}
\end{array}\right] \underline{\mathbf{a}}=\left[\begin{array}{c}
\underline{\mathbf{z}}_{l}\left(0^{+}\right) \\
\underline{\mathbf{z}}_{(l+1)_{z_{l+1}}}^{*}\left(0^{+}\right) \\
\underline{\mathbf{z}}_{(l+2)_{z 2}}^{*}\left(0^{+}\right) \\
\vdots \\
\\
\underline{\mathbf{z}}_{\kappa_{z_{\kappa}}}^{*}\left(0^{+}\right)
\end{array}\right] }
\end{aligned}
$$

or equivalently,

$$
\left[\begin{array}{c}
\mathbf{V}_{l} \\
\mathbf{V}_{(l+1)_{z_{l+1}}}^{*} \\
\mathbf{V}_{(l+2)_{z_{l+2}}}^{*} \\
\vdots \\
\mathbf{V}_{\mathcal{\kappa}_{d_{\kappa}}}^{*}
\end{array}\right] \underline{\mathbf{a}}=\left[\begin{array}{c}
\underline{\mathbf{z}}_{l}^{*}\left(0^{+}\right) \\
\underline{\mathbf{z}}_{(l+1)_{z_{l+1}}}^{*}\left(0^{+}\right) \\
\underline{\mathbf{z}}_{(l+2)_{z 2}}^{*}\left(0^{+}\right) \\
\vdots \\
\underline{\mathbf{z}}_{z_{z_{\kappa}}}^{*}\left(0^{+}\right)
\end{array}\right],
$$

where $\underline{\mathbf{z}}_{l}^{*}\left(0^{+}\right)=\left(1 / \Phi^{-1}(K)\right)(\underbrace{1 / b_{\tau_{1}} \bigoplus 1 / b_{\tau_{2}} \bigoplus \cdots \bigoplus 1 / b_{\tau_{l}}}_{l})$ $\underline{\mathbf{z}}_{l}\left(0^{+}\right)$, since $\left(b_{\tau_{1}} \oplus b_{\tau_{2}} \oplus \cdots \oplus b_{\tau_{l}}\right)$ is nonsingular. 
Remark 3. The matrices given by (67) with some row transformations can be transformed to the following:

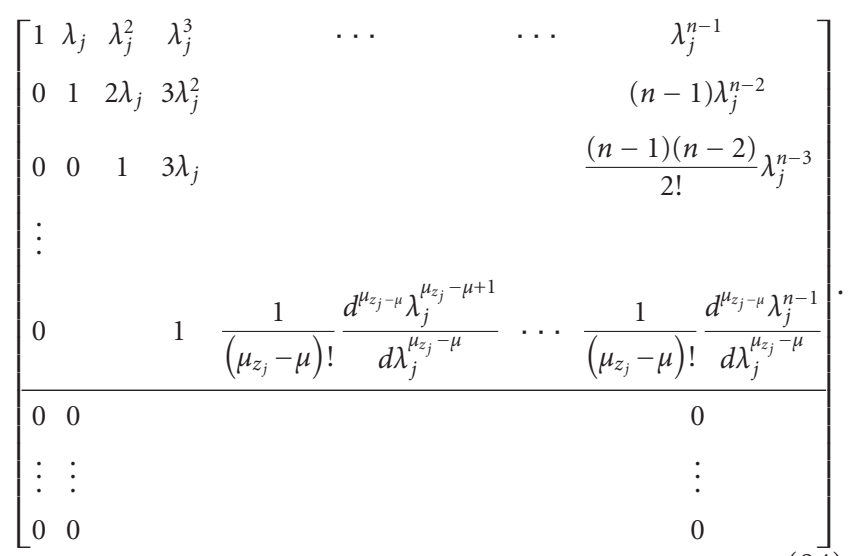

The matrix (84) is denoted by $\mathbf{V}_{j_{z_{j}}}^{* *}$. Thus, there is a nonsingular matrix $\Lambda_{j_{z_{j}}}$ such as

$$
\Lambda_{j_{z_{j}}} \mathbf{V}_{j_{z_{j}}}^{*}=\mathbf{V}_{j_{z_{j}}}^{* *}
$$

Then, subsystems (74) are transformed to

$$
\Lambda_{j_{z_{j}}} \mathbf{V}_{j_{z_{j}}}^{*} \underline{\mathbf{a}}=\Lambda_{j_{z_{j}}} \underline{\mathbf{z}}_{j_{j}}^{*}\left(0^{+}\right) \Longleftrightarrow \mathbf{V}_{j_{z_{j}}}^{* *} \underline{\mathbf{a}}=\underline{\mathbf{z}}_{j_{z_{j}}}^{* *}\left(0^{+}\right)
$$

where $\Lambda_{j_{z_{j}}} \underline{\mathbf{z}}_{j_{j}}^{*}\left(0^{+}\right)=\underline{\mathbf{z}}_{j_{j}}^{* *}\left(0^{+}\right)$for every $z_{j}=1,2, \ldots, d_{j}$, and $j=l+1, l+2, \ldots, \kappa$. Consequently, system (83) is transformed to

$$
\left[\begin{array}{c}
\mathbf{V}_{l} \\
\mathbf{V}_{(l+1)_{z_{l+1}}}^{* *} \\
\mathbf{V}_{(l+2)_{z_{l+2}}^{* *}} \\
\vdots \\
\mathbf{V}_{\kappa_{d_{k}}}^{* *}
\end{array}\right] \underline{\mathbf{a}}=\left[\begin{array}{c}
\underline{\mathbf{z}}_{l}^{*}\left(0^{+}\right) \\
\underline{\mathbf{z}}_{(l+1)_{z_{l+1}}}^{* *}\left(0^{+}\right) \\
\underline{\mathbf{z}}_{(l+2)_{z 2}}^{* *}\left(0^{+}\right) \\
\vdots \\
\underline{\mathbf{z}}_{\kappa_{z_{\kappa}}}^{* *}\left(0^{+}\right)
\end{array}\right]
$$

Comment 1. Without loss of generality, we can assume that the matrices $\mathbf{V}_{j_{d_{j}}}^{* *}$ do not contain zero rows. On contrary, if there exist some zero rows, the solvability of system (87) is not affected; only the number of nonzero rows which are included in (87) is changed.

Remark 4. System (87) can be further transposed to a more convenient system. Analytically, if we multiply the 1st row of Vandermonde matrix $\mathbf{V}_{l}$, that is, $\left[\begin{array}{lllll}1 & \lambda_{1} & \lambda_{1}^{2} & \cdots & \lambda_{1}^{n-1}\end{array}\right]$ with the number $(-1)$ and it is added to the 1st row of each of $\mathbf{V}_{(l+1)_{z_{l+1}}^{* *}}^{*}, \mathbf{V}_{(l+2)_{z_{l+2}}^{* *}}^{*}, \ldots, \mathbf{V}_{\kappa_{z_{\kappa}}}^{* *}$, then $\mathbf{V}_{j_{z_{j}}}^{* *}$ is given by

$$
\left[\begin{array}{ccccc}
0 & \lambda_{j}-\lambda_{1} & \lambda_{j}^{2}-\lambda_{1}^{2} & \cdots & \lambda_{j}^{n-1}-\lambda_{1}^{n-1} \\
0 & 1 & 2 \lambda_{j} & \cdots & (n-1) \lambda_{j}^{n-2} \\
\vdots & 1 & & \\
\vdots & & & \ddots & \\
0 & 0 & \cdots & 0 & 1, \frac{1}{\left(\rho_{j}-1\right) !} \frac{d^{\rho_{j}-1} \lambda_{j}^{\rho_{j+1}}}{d \lambda_{j}^{\rho_{j}-1}}, \ldots, \frac{1}{\left(\rho_{j}-1\right) !} \frac{d^{\rho_{j}-1} \lambda_{j}^{n-1}}{d \lambda_{j}^{\rho_{j}-1}}
\end{array}\right] .
$$

(Note that we have assumed that the matrices $\mathbf{V}_{j_{j}}^{* *}$, for $j=$ $l+1, l+2, \ldots, \kappa$, do not cotain zero rows; see also Comment)

We can easily see that the 1st row of matrix (88) can be rewritten as what follows.

The element $\lambda_{j}^{\kappa}+\lambda_{j}^{\kappa-1} \lambda_{1}+\cdots+\lambda_{j} \lambda_{1}^{\kappa-1}+\lambda_{1}^{\kappa}=$ $\sum_{m_{1}, m_{2}=0, \sum_{i=1}^{2} m_{i}=\kappa}^{\kappa} \lambda_{j}^{m_{1}} \lambda_{1}^{m_{2}}$.

Thus, the first row is presented as

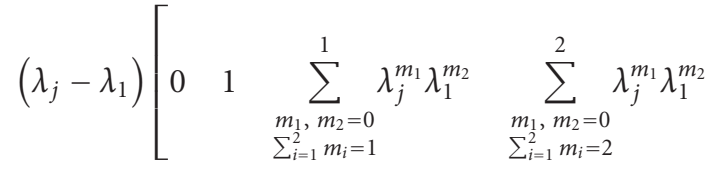

$$
\begin{aligned}
& \left.\ldots \quad \sum_{\substack{m_{1}, m_{2}=0 \\
\sum_{i=1}^{2} m_{i}=n-2}}^{n-2} \lambda_{j}^{m_{1}} \lambda_{1}^{m_{2}}\right] \text {. }
\end{aligned}
$$

Since, the element $\lambda_{j}-\lambda_{1} \neq 0$, we can multiply by left the equation (88) with a properly chosen transformation matrix, so as to obtain

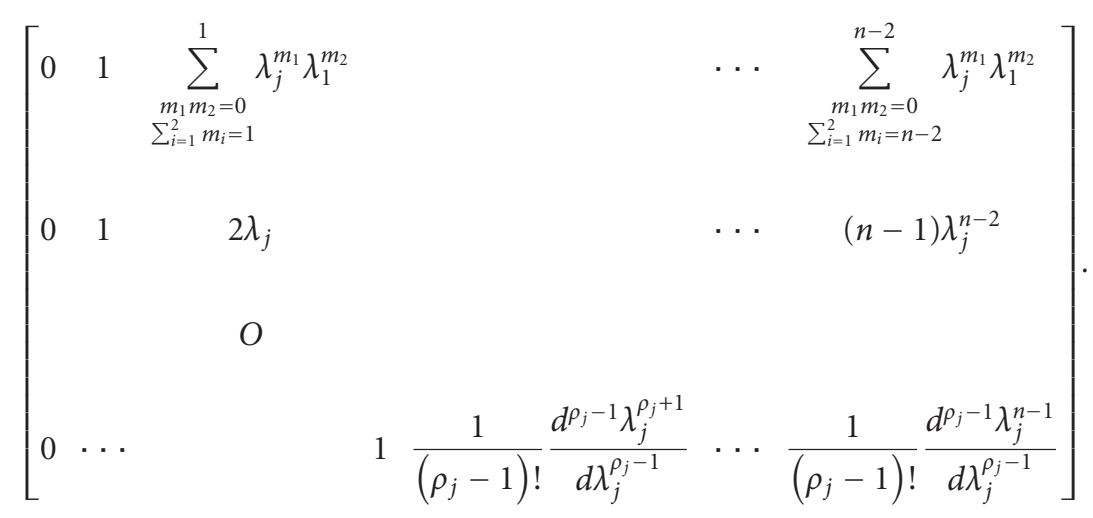


Finally, this subsection is concluded by considering system (91), where the matrices $\mathbf{A}_{j_{j}}$ for $j=l+1, l+2, \ldots, \kappa$ are derived by taking into account a properly chosen transformation left-matrix $\mathrm{Z}$ as follows:

$$
Z\left[\begin{array}{c}
\mathbf{V}_{l} \\
\mathbf{V}_{(l+1)_{z_{l+1}}}^{* *} \\
\mathbf{V}_{(l+2)_{z_{l+2}} *}^{*} \\
\vdots \\
\mathbf{V}_{\mathcal{\kappa}_{d_{\kappa}}}^{* *}
\end{array}\right] \underline{\mathbf{a}}=Z\left[\begin{array}{c}
\underline{\mathbf{z}}_{l}^{*}\left(0^{+}\right) \\
\underline{\mathbf{z}}_{(l+1)_{z_{l+1}} *}^{* *}\left(0^{+}\right) \\
\underline{\mathbf{z}}_{(l+2)_{z 2}}^{* *}\left(0^{+}\right) \\
\vdots \\
\underline{\mathbf{z}}_{\kappa_{z_{\kappa}}}^{* *}\left(0^{+}\right)
\end{array}\right] \Longleftrightarrow\left[\begin{array}{c}
\mathbf{V}_{l} \\
\mathbf{A}_{l+1} \\
\mathbf{A}_{l+2} \\
\vdots \\
\mathbf{A}_{\kappa}
\end{array}\right] \underline{\mathbf{a}}=\left[\begin{array}{c}
\underline{\mathbf{z}}_{l}^{*}\left(0^{+}\right) \\
\underline{\mathbf{d}}_{l+1}\left(0^{+}\right) \\
\underline{\mathbf{d}}_{l+2}\left(0^{+}\right) \\
\vdots \\
\underline{\mathbf{d}}_{\kappa}\left(0^{+}\right)
\end{array}\right]
$$

where

$$
\left[\begin{array}{c}
\underline{\mathbf{z}}_{l}^{*}\left(0^{+}\right) \\
\underline{\mathbf{d}}_{l+1}\left(0^{+}\right) \\
\underline{\mathbf{d}}_{l+2}\left(0^{+}\right) \\
\vdots \\
\underline{\mathbf{d}}_{\kappa}\left(0^{+}\right)
\end{array}\right]=Z\left[\begin{array}{c}
\underline{\mathbf{z}}_{l}^{*}\left(0^{+}\right) \\
\underline{\mathbf{z}}_{(l+1)_{z_{l+1}} *}^{* *}\left(0^{+}\right) \\
\underline{\mathbf{z}}_{(l+2)_{z 2}}^{* *}\left(0^{+}\right) \\
\vdots \\
\underline{\mathbf{z}}_{\kappa_{z_{\kappa}}}^{* *}\left(0^{+}\right)
\end{array}\right]
$$

with

$$
\begin{gathered}
\mathbf{V}_{l} \in \mathcal{M}_{\ln }, \quad \mathbf{V}_{j} \in \mathcal{M}_{j n} \\
\text { for } j=l+1, l+2, \ldots, \kappa, \\
\underline{\mathbf{z}}_{l}^{*}\left(0^{+}\right) \in \mathcal{M}_{l 1}, \quad \underline{\mathbf{d}}_{l+2}\left(0^{+}\right) \in \mathcal{M}_{j 1}, \\
\text { for } j=l+1, l+2, \ldots, \kappa .
\end{gathered}
$$

$\rho_{j}=\max _{z_{i}=1,2, \ldots, d_{j}} \mu_{z_{j}}$ is the index of annihilation for the eigenvalue $\lambda_{j}$.

Consequently, the system (91) contains the following subsystems

$$
\left.\begin{array}{c}
\mathbf{V}_{l \underline{\mathbf{a}}}=\underline{\mathbf{z}}_{l}^{*}\left(0^{+}\right), \\
\mathbf{A}_{l+1} \underline{\mathbf{a}}=\underline{\mathbf{d}}_{l+1}\left(0^{+}\right), \\
\ldots \\
\mathbf{A}_{\kappa} \underline{\mathbf{a}}=\underline{\mathbf{d}}_{\kappa}\left(0^{+}\right)
\end{array}\right\},
$$

where $\mathbf{V}_{l}, \mathbf{A}_{j}$ for $j=l+1, l+2, \ldots, \kappa$ are nonsquare matrices.

In order to solve system (91), we should use the relevant theory of generalized inverses. Some basic elements are briefly presented in the next subsection.

3.3. Solving Subsystem (91). This subsection is beginning with the brief presentation of the most basic results of $\{1\}-$ generalized inverses.

Definition 12. For a matrix $A \in \mathcal{M}_{p q}$, the $X \in \mathcal{M}_{q p}$ is called $\{1\}$-inverses of matrix $A$, if

$$
A X A=A .
$$

The following proposition provides the means for finding $\{1\}$-inverses; see $[19,20]$ for proofs and details.
Proposition 13. Let $A \in \mathcal{M}_{p q}$, with $\operatorname{rank} A=r<\min \{p, q\}$ and $P \in \mathcal{M}_{p}, Q \in \mathcal{M}_{q}$, invertible such that

$$
P A Q=I_{r} \bigoplus \mathbb{O}_{p-r, q-r}
$$

Then, every $X,\{1\}$-inverse of matrix $A$, is written as follows:

$$
X=Q\left(I_{r} \bigoplus \mathbb{O}_{p-r, q-r}\right) P
$$

for arbitrary $L \in \mathcal{M}_{(q-r)(p-r)}$.

Definition 14. We define any $\{1\}$-inverse of matrix $A$ by $A^{(1)}$.

The following result is due to [6].

Theorem 15. Let $A \in \mathcal{M}_{p q}, B \in \mathcal{M}_{d v}, D \in \mathcal{M}_{p v}$. Then the matrix equation

$$
A X B=D,
$$

is consistent if and only if for some $A^{(1)}, B^{(1)}$

$$
A A^{(1)} D B^{(1)} B=D
$$

and the general solution is given by

$$
X=A^{(1)} D B^{(1)}+Y-A^{(1)} A Y B B^{(1)}
$$

for arbitrary $Y \in \mathcal{M}_{p d}$. [20].

The following characterization of the set of $A^{(1)}$ is due to

Corollary 16. Let $A \in \mathcal{M}_{p q}$ and $A^{(1)} \in \mathcal{M}_{q p}$, then any other $\{1\}$-inverse $\tilde{A}^{(1)} \in \mathcal{M}_{q p}$ of matrix $A$ is given by

$$
\tilde{A}^{(1)}=A^{(1)}+Z-A^{(1)} A Z A A^{(1)}
$$

for some $Z \in \mathcal{M}_{q p}$.

A special case of $\{1\}$-inverses is the left/right inverses of a full column/row rank matrix, respectively. The proofs and further details are presented extensively by $[19,20]$.

Definition 17. Consider the following matrices.

(a) Let $P_{i}(\alpha)$ be a $m \times m$-matrix

$$
P_{i}(\alpha)=\left[\begin{array}{lllllll}
1 & & & & & & 0 \\
& 1 & & & & & \\
& & \ddots & & & & \\
& & & \alpha & & & \\
& & & 1 & & \\
& & & & & & \\
& & & & & & \\
\mathbb{O} & & & & & & 1
\end{array}\right] .
$$

(The nonzero element $a$ is in the ith row and the ith column.)

Thus, whenever a matrix $A$ is multiplied from the left by $P_{i}(\alpha)$, then its $i$ th row is multiplied by the nonzero number $a$. 
(b) Let $P_{i}(j, a)$ be an $m \times m$-matrix

$$
P_{i}(j, \alpha)=\left[\begin{array}{cccccccc}
1 & & & & & & & 0 \\
& 1 & & & & & & \\
& & \ddots & & & & & \\
& & 1 & \cdots & \alpha & \\
& & & \ddots & \vdots & \\
& & & & 1 & \\
& & & & & & \\
& & & & & \ddots & \\
0 & & & & & & 1
\end{array}\right] .
$$

(The nonzero element $a$ is in the $i$ th row and the $j$ th column.)

Thus, whenever a matrix $A$ is multiplied from the left by $P_{i}(j, a)$, then its $j$ th row is multiplied by the nonzero number $a$ and it is added to the $j$ th row of $A$.

(c) Let $Q_{i}(a)$ be an $n \times n$-matrix

$$
Q_{i}(\alpha)=\left[\begin{array}{llllllll}
1 & & & & & & \\
& 1 & & & & & \\
& & \ddots & & & & \\
& & & 1 & & & \\
& & & \alpha & & \\
& & & & 1 & & \\
& & & & & \ddots & \\
& & & & & & \\
& & & & & & 1
\end{array}\right] .
$$

(The nonzero element $a$ is in the $i$ th-row and the $i$ thcolumn).

Thus, whenever a matrix $A$ is multiplied from the right by $Q_{i}(\alpha)$, then its $i$ th column is multiplied by the nonzero number $a$.

(d) Let $Q_{i}(j, a)$ be an $n \times n$-matrix

$$
Q_{i}(j, \alpha)=\left[\begin{array}{llllllll}
1 & & & & & & \\
& 1 & & & & & & \\
& & \ddots & & & & & \\
& & 1 & & & & \\
& & \vdots & 1 & & & \\
& & & & \ddots & & \\
& & \alpha & \ldots & & 1 & \\
& & & & & & \ddots \\
& & & & & & & \\
& & & & & & & 1
\end{array}\right] .
$$

(The nonzero element $a$ is in the $j$ th row and the $i$ th column.)

Thus, whenever a matrix $A$ is multiplied from the right by $Q_{i}(j, a)$, then its $i$ th column is multiplied by the nonzero number $a$ and it is added to the $j$ th column of $A$.

Now, in order to calculate the generalized inverses of Vandermonde and the relative to it matrices, we use the recent results of paper [6]. In this context, the following propositions are relevant.

Proposition 18. The $\{1\}$-inverse of the Vandermonde matrix is given by

$$
\mathbf{V}_{l}^{(1)}=\mathbf{Q}\left[\begin{array}{c}
I_{l} \\
\mathbb{O}_{n-l, l}
\end{array}\right] \mathbf{P}
$$

where

$$
\mathbf{P}=\prod_{s=l-1}^{1} \prod_{j=l}^{s+1} P_{j}\left(\frac{1}{\lambda_{j}-\lambda_{s}}\right) P_{j}(s,-1) \in \mathcal{M}_{l},
$$

(the multiplication counts backwards, starting from $l \rightarrow l-$ $1 \rightarrow \cdots$ etc.)

$$
\mathbf{Q}=\prod_{s=1}^{l} \prod_{r=s+1}^{n} Q_{r}\left(s,-\sum_{\substack{m_{1}, \ldots, m_{s}=0 \\ m_{1}+\cdots+m_{s}=r-s}}^{r-s} \prod_{\zeta=1}^{s} \lambda_{\zeta}^{m_{\zeta}}\right) \in \mathcal{M}_{n},
$$

and the general solution of system $\mathbf{V}_{l} \underline{\mathbf{a}}=\underline{\mathbf{z}}_{l}^{*}\left(0^{+}\right)$is given by

$$
\underline{\mathbf{a}}=\mathbf{V}_{l}^{(1)} \underline{\mathbf{z}}_{l}^{*}\left(0^{+}\right)+\left(I_{n}-\mathbf{V}_{l}^{(1)} \mathbf{V}_{l}\right) \underline{\psi},
$$

for an arbitrarily chosen vector $\psi \in \mathcal{M}_{n 1}$.

Proof. The proof is a straightforward application of Theorem 15. Analytically,

$$
\mathbf{P V}_{l} \mathbf{Q}=\left[\begin{array}{ll}
I_{l} & \mathbb{O}_{l, n-l}
\end{array}\right] \Longleftrightarrow \mathbf{V}_{l}=\mathbf{P}^{-1}\left[\begin{array}{ll}
I_{l} & \mathbb{O}_{l, n-l}
\end{array}\right] \mathbf{Q}^{-1}
$$

In order for the matrix $\mathbf{Q}\left[\begin{array}{c}I_{l} \\ \mathbb{O}_{n-l, l}\end{array}\right] \mathbf{P}$ to be the $\{1\}$-inverse of $\mathbf{V}_{l}$, we should prove the following equality

$$
\mathbf{V}_{l} \mathbf{Q}\left[\begin{array}{c}
I_{l} \\
\mathbb{O}_{n-l, l}
\end{array}\right] \mathbf{P V}_{l}=\mathbf{V}_{l}
$$

Thus,

$$
\begin{aligned}
\mathbf{V}_{l} \mathbf{Q}\left[\begin{array}{c}
I_{l} \\
\mathbb{O}_{n-l, l}
\end{array}\right] \mathbf{P} \mathbf{V}_{l} \\
=\mathbf{P}^{-1}\left[\begin{array}{ll}
I_{l} & \mathbb{O}_{l, n-l}
\end{array}\right] \mathbf{Q}^{-1} \mathbf{Q}\left[\begin{array}{c}
I_{l} \\
\mathbb{O}_{n-l, l}
\end{array}\right] \mathbf{P P}^{-1}\left[\begin{array}{ll}
I_{l} & \mathbb{O}_{l, n-l}
\end{array}\right] \mathbf{Q}^{-1} \\
= \\
=\mathbf{P}^{-1}\left[\begin{array}{ll}
I_{l} & \mathbb{O}_{l, n-l}
\end{array}\right]\left[\begin{array}{c}
I_{l} \\
\mathbb{O}_{n-l, l}
\end{array}\right]\left[\begin{array}{ll}
I_{l} & \mathbb{O}_{l, n-l}
\end{array}\right] \mathbf{Q}^{-1} \\
= \\
=\mathbf{P}^{-1}\left(I_{l} \cdot I_{l}+\mathbb{O}_{l, n-l} \cdot \mathbb{O}_{n-l, l}\right)\left[\begin{array}{ll}
I_{l} & \mathbb{O}_{l, n-l}
\end{array}\right] \mathbf{Q}^{-1} \\
=\mathbf{P}^{-1}\left[\begin{array}{ll}
I_{l} & \mathbb{O}_{l, n-l}
\end{array}\right] \mathbf{Q}^{-1}=\mathbf{V}_{l} .
\end{aligned}
$$

Hence, the solution of system $\mathbf{V}_{l} \underline{\mathbf{a}}=\underline{\mathbf{z}}_{l}^{*}\left(0^{+}\right)$is given by (113). 
Proposition 19. The $\{1\}$-inverse of the matrix $\mathbf{A}_{l}$ for $j=l+$ $1, l+2, \ldots, \kappa$ is given by

$$
\mathbf{A}_{j}^{(1)}=\mathbf{Q}_{j}\left[\begin{array}{c}
\underline{0}_{p_{j}}^{t} \\
I_{p_{j}} \\
\mathbb{O}_{n-p_{j}-1, p_{j}}
\end{array}\right] \mathbf{P}_{j},
$$

where

$$
\begin{gathered}
\mathbf{P}_{j}=\prod_{s=2}^{p_{j}} P_{p_{j}-s+2}\left(\frac{1}{\lambda_{j}-\lambda_{1}}\right) P_{p_{j}-s+2}\left(p_{j}-s+1,-1\right), \\
\mathbf{Q}_{j}=\prod_{s=2}^{p_{j}+1} \prod_{m=s+1}^{n} Q_{m}\left(s,-\frac{1}{(s-2) !} \frac{d^{s-2}}{d \lambda_{j}^{s-2}}\left(\sum_{\substack{m_{1}, m_{2}=0 \\
m_{1}+m_{2}=m-2}}^{m-2} \lambda_{1}^{m_{1}} \lambda_{j}^{m_{2}}\right)\right) .
\end{gathered}
$$

for $j=l+1, l+2, \ldots, \kappa$, and the general solution of systems $\mathbf{A}_{j} \underline{\mathbf{a}}=\underline{\mathbf{d}}_{j}\left(0^{+}\right)$is given by

$$
\underline{\mathbf{a}}=\mathbf{A}_{j}^{(1)} \underline{\mathbf{d}}_{j}\left(0^{+}\right)+\left(I_{n}-\mathbf{A}_{j}^{(1)} \mathbf{A}_{j}\right) \underline{\psi}_{j}
$$

for an arbitrarily chosen vector $\underline{\psi}_{j} \in \mathcal{M}_{n 1}$ for $j=l+1, l+$ $2, \ldots, \kappa$.

Proof. The proof is also a straightforward application of Theorem 15. Analytically,

$$
\begin{aligned}
\mathbf{P}_{j} \mathbf{A}_{j} \mathbf{Q}_{j} & =\left[\begin{array}{lll}
\underline{0}_{p_{j}} & I_{p_{j}} & \mathbb{O}_{p_{j}, n-p_{j}-1}
\end{array}\right] \\
& \Longleftrightarrow \mathbf{A}_{j}=\mathbf{P}_{j}^{-1}\left[\begin{array}{lll}
\underline{0}_{p_{j}} & I_{p_{j}} & \mathbb{O}_{p_{j}, n-p_{j}-1}
\end{array}\right] \mathbf{Q}_{j}^{-1} .
\end{aligned}
$$

In order for the matrix

$$
\mathbf{Q}_{j}\left[\begin{array}{c}
\underline{0}_{p_{j}}^{t} \\
I_{p_{j}} \\
\mathbb{O}_{n-p_{j}-1, p_{j}}
\end{array}\right] \mathbf{P}_{j}
$$

to be the $\{1\}$-inverse of $\mathbf{A}_{j}$, we should prove the following equality:

$$
\mathbf{A}_{j} \mathbf{Q}_{j}\left[\begin{array}{c}
\underline{0}_{p_{j}}^{t} \\
I_{p_{j}} \\
\mathbb{O}_{n-p_{j}-1, p_{j}}
\end{array}\right] \mathbf{P}_{j} \mathbf{A}_{j}=\mathbf{A}_{j}
$$

Thus,

$$
\begin{aligned}
& \mathbf{A}_{j} \mathbf{Q}_{j}\left[\begin{array}{c}
\underline{0}_{p_{j}}^{t} \\
I_{p_{j}} \\
\mathbb{O}_{n-p_{j}-1, p_{j}}
\end{array}\right] \mathbf{P}_{j} \mathbf{A}_{j} \\
&=\mathbf{P}_{j}^{-1}\left[\begin{array}{lll}
\underline{0}_{p_{j}} & I_{p_{j}} & \mathbb{O}_{p_{j}, n-p_{j}-1}
\end{array}\right] \mathbf{Q}_{j}^{-1} \mathbf{Q}_{j}\left[\begin{array}{c}
\underline{0}_{p_{j}}^{t} \\
I_{p_{j}} \\
\mathbb{O}_{n-p_{j}-1, p_{j}}
\end{array}\right] \mathbf{P}_{j} \mathbf{P}_{j}^{-1} \\
& \\
& \times\left[\begin{array}{lll}
\underline{0}_{p_{j}} & I_{p_{j}} & \mathbb{O}_{p_{j}, n-p_{j}-1}
\end{array}\right] \mathbf{Q}_{j}^{-1} \\
&= \mathbf{P}_{j}^{-1}\left[\begin{array}{lll}
\underline{0}_{p_{j}} & I_{p_{j}} & \mathbb{O}_{p_{j}, n-p_{j}-1}
\end{array}\right]\left[\begin{array}{c}
\underline{0}_{p_{j}}^{t} \\
I_{p_{j}} \\
\mathbb{O}_{n-p_{j}-1, p_{j}}
\end{array}\right] \\
& \times\left[\begin{array}{lll}
\underline{0}_{p_{j}} & I_{p_{j}} & \mathbb{O}_{p_{j}, n-p_{j}-1}
\end{array}\right] \mathbf{Q}_{j}^{-1} \\
&= \mathbf{P}_{j}^{-1}\left(\begin{array}{lll}
\underline{0}_{p_{j}} & \underline{0}_{p_{j}}^{t}+I_{p_{j}} \cdot I_{p_{j}}+\mathbb{O}_{p_{j}, n-p_{j}-1} \cdot \mathbb{O}_{n-p_{j}-1, p_{j}}
\end{array}\right] \\
& \times\left[\begin{array}{lll}
\underline{0}_{p_{j}} & I_{p_{j}} & \mathbb{O}_{p_{j}, n-p_{j}-1}
\end{array}\right] \mathbf{Q}_{j}^{-1} \\
&= \mathbf{P}_{j}^{-1}\left[\begin{array}{lll}
\underline{0}_{p_{j}} & I_{p_{j}} & \mathbb{O}_{p_{j}, n-p_{j}-1}
\end{array}\right] \mathbf{Q}_{j}^{-1}=\mathbf{A}_{j} .
\end{aligned}
$$

Hence, the solution of system $\mathbf{A}_{j} \underline{\mathbf{a}}=\underline{\mathbf{d}}_{j}\left(0^{+}\right)$is given by (115).

3.4. The Solution of System (91). This subsection concludes the whole discussion. The following theorem holds.

Theorem 20. System (91) is solvable, that is,

$$
A \underline{\mathbf{a}}=\underline{\mathbf{b}},
$$

where

$$
\mathbf{A} \triangleq\left[\begin{array}{c}
\mathbf{V}_{l} \\
\mathbf{A}_{l+1} \\
\mathbf{A}_{l+2} \\
\vdots \\
\mathbf{A}_{\kappa}
\end{array}\right], \quad \underline{\mathbf{b}} \triangleq\left[\begin{array}{c}
\underline{\mathbf{z}}_{l}^{*}\left(0^{+}\right) \\
\underline{\mathbf{d}}_{l+1}\left(0^{+}\right) \\
\underline{\mathbf{d}}_{l+2}\left(0^{+}\right) \\
\vdots \\
\underline{\mathbf{d}}_{\kappa}\left(0^{+}\right)
\end{array}\right]
$$

if the following conditions are satisfied:

$$
\begin{aligned}
\mathbf{V}_{l}\left[\mathbf{A}_{j}^{(1)} \underline{\mathbf{d}}_{j}\left(0^{+}\right)+\left(I_{n}-\mathbf{A}_{j}^{(1)} \mathbf{A}_{j}\right) \underline{\psi}\right] & =\underline{\mathbf{d}}_{l}, \\
\mathbf{A}_{j}\left[\mathbf{V}_{l}^{(1)} \underline{\mathbf{z}}_{l}\left(0^{+}\right)+\left(I_{n}-\mathbf{V}_{l}^{(1)} \mathbf{V}_{l}\right) \underline{\psi}\right] & =\underline{\mathbf{d}}_{j}, \\
\mathbf{A}_{i}\left[\mathbf{A}_{j}^{(1)} \underline{\mathbf{d}}_{j}\left(0^{+}\right)+\left(I_{n}-\mathbf{A}_{j}^{(1)} \mathbf{A}_{j}\right) \underline{\psi}\right] & =\underline{\mathbf{d}}_{i}
\end{aligned}
$$

for $i \neq j$ and $i, j=l+1, l+2, \ldots, \kappa$. 
Proof. Following Propositions 18 and 19, we have

(i) for system $\mathbf{V}_{l} \underline{\mathbf{a}}=\underline{\mathbf{z}}_{l}^{*}\left(0^{+}\right)$, the solution is given by $\underline{\mathbf{a}}=$ $\mathbf{V}_{l}^{(1)} \underline{\mathbf{z}}_{l}^{*}\left(0^{+}\right)+\left(I_{n}-\mathbf{V}_{l}^{(1)} \mathbf{V}_{l}\right) \psi$,

(ii) for systems $\mathbf{A}_{j} \underline{\mathbf{a}}=\underline{\mathbf{d}}_{j}\left(0^{+}\right)$, the solution is given by $\underline{\mathbf{a}}=\mathbf{A}_{j}^{(1)} \underline{\mathbf{d}}_{j}\left(0^{+}\right)+\left(I_{n}-\mathbf{A}_{j}^{(1)} \mathbf{A}_{j}\right) \psi$ for arbitrarily chosen vector $\psi \in \mathcal{M}_{n 1}$ for $j=l+1, \bar{l}+2, \ldots, \kappa$.

There are infinitely many solutions, since the vector $\psi \in$ $\mathcal{M}_{n 1}$ is arbitrary. Moreover, each of the above subsystems also contains the solution of system $\mathbf{A} \underline{\mathbf{a}}=\underline{\mathbf{b}}$. Thus, we assume that the desired solution is given by $\mathbf{a}$, then it should also satisfy the first system, that is, $\mathbf{V}_{l} \underline{\mathbf{a}}=\underline{\mathbf{z}}_{l}^{*}\left(0^{+}\right)$. Thus, we take

$$
\begin{aligned}
& \underline{\mathbf{a}}=\mathbf{V}_{l}^{(1)} \underline{\mathbf{z}}_{l}^{*}\left(0^{+}\right)+\left(I_{n}-\mathbf{V}_{l}^{(1)} \mathbf{V}_{l}\right) \underline{\psi}, \\
& \mathbf{A} \underline{\mathbf{a}}=\underline{\mathbf{b}} \Longleftrightarrow \mathbf{A}\left(\mathbf{V}_{l}^{(1)} \underline{\mathbf{z}}_{l}^{*}\left(0^{+}\right)+\left(I_{n}-\mathbf{V}_{l}^{(1)} \mathbf{V}_{l}\right) \underline{\psi}\right)=\underline{\mathbf{b}} \\
& \Leftrightarrow \mathbf{A V}_{l}^{(1)} \underline{\mathbf{z}}_{l}^{*}\left(0^{+}\right)+\mathbf{A}\left(I_{n}-\mathbf{V}_{l}^{(1)} \mathbf{V}_{l}\right) \underline{\psi}=\underline{\mathbf{b}} \\
& \Leftrightarrow \mathbf{A V}_{l}^{(1)} \underline{\mathbf{z}}_{l}^{*}\left(0^{+}\right)+\left(\mathbf{A}-\mathbf{A} \mathbf{V}_{l}^{(1)} \mathbf{V}_{l}\right) \underline{\psi}=\underline{\mathbf{b}} \\
& \Leftrightarrow\left[\begin{array}{c}
\mathbf{V}_{l} \\
\mathbf{A}_{l+1} \\
\mathbf{A}_{l+2} \\
\vdots \\
\mathbf{A}_{\kappa}
\end{array}\right] \mathbf{V}_{l}^{(1)} \underline{\mathbf{z}}_{l}^{*}\left(0^{+}\right)+\left(\left[\begin{array}{c}
\mathbf{V}_{l} \\
\mathbf{A}_{l+1} \\
\mathbf{A}_{l+2} \\
\vdots \\
\mathbf{A}_{\kappa}
\end{array}\right]-\left[\begin{array}{c}
\mathbf{V}_{l} \\
\mathbf{A}_{l+1} \\
\mathbf{A}_{l+2} \\
\vdots \\
\mathbf{A}_{\kappa}
\end{array}\right] V_{l}^{(1)} V_{l}\right) \underline{\psi} \\
& \triangleq\left[\begin{array}{c}
\underline{\mathbf{z}}_{l}^{*}\left(0^{+}\right) \\
\underline{\mathbf{d}}_{l+1}\left(0^{+}\right) \\
\underline{\mathbf{d}}_{l+2}\left(0^{+}\right) \\
\vdots \\
\underline{\mathbf{d}}_{\kappa}\left(0^{+}\right)
\end{array}\right] \Leftrightarrow\left[\begin{array}{c}
\underline{\mathbf{z}}_{l}^{*}\left(0^{+}\right) \\
\mathbf{A}_{l+1} \mathbf{V}_{l}^{(1)} \underline{\mathbf{z}}_{l}^{*}\left(0^{+}\right) \\
\mathbf{A}_{l+2} \mathbf{V}_{l}^{(1)} \underline{\mathbf{z}}_{l}^{*}\left(0^{+}\right) \\
\vdots \\
\mathbf{A}_{\kappa} \mathbf{V}_{l}^{(1)} \underline{\mathbf{z}}_{l}^{*}\left(0^{+}\right)
\end{array}\right] \\
& +\left[\begin{array}{c}
\mathbb{O}_{l} \\
\mathbf{A}_{l+1}\left(I_{n}-\mathbf{V}_{l}^{(1)} \mathbf{V}_{l}\right) \\
\mathbf{A}_{l+2}\left(I_{n}-\mathbf{V}_{l}^{(1)} \mathbf{V}_{l}\right) \\
\vdots \\
\mathbf{A}_{\kappa}\left(I_{n}-\mathbf{V}_{l}^{(1)} \mathbf{V}_{l}\right)
\end{array}\right] \underline{\psi}=\left[\begin{array}{c}
\underline{\mathbf{z}}_{l}^{*}\left(0^{+}\right) \\
\underline{\mathbf{d}}_{l+1}\left(0^{+}\right) \\
\underline{\mathbf{d}}_{l+2}\left(0^{+}\right) \\
\vdots \\
\underline{\mathbf{d}}_{\kappa}\left(0^{+}\right)
\end{array}\right] \\
& \Leftrightarrow \mathbf{A}_{j} \mathbf{V}_{l}^{(1)} \underline{\mathbf{z}}_{l}^{*}\left(0^{+}\right)+\mathbf{A}_{j}\left(I_{n}-\mathbf{V}_{l}^{(1)} \mathbf{V}_{l}\right) \underline{\psi}=\underline{\mathbf{d}}_{j}\left(0^{+}\right) \\
& \Leftrightarrow \mathbf{A}_{j}\left[\mathbf{V}_{l}^{(1)} \underline{\mathbf{z}}_{l}^{*}\left(0^{+}\right)+\left(I_{n}-\mathbf{V}_{l}^{(1)} \mathbf{V}_{l}\right) \underline{\psi}\right]=\underline{\mathbf{d}}_{j}\left(0^{+}\right)
\end{aligned}
$$

for every $j=l+1, l+2 \ldots, \kappa$. So (123) has been derived. Analogously, the system $\mathbf{A} \underline{\mathbf{a}}=\underline{\mathbf{b}}$ should also satisfy every subsystem $\mathbf{A}_{j} \underline{\mathbf{a}}=\underline{\mathbf{d}}_{j}\left(0^{+}\right)$for $j=l+1, l+2, \ldots, \kappa$.
Consequently, we have that

$$
\begin{aligned}
& \underline{\mathbf{a}}=\mathbf{A}_{j}^{(1)} \underline{\mathbf{d}}_{j}\left(0^{+}\right)+\left(I_{n}-\mathbf{A}_{j}^{(1)} \mathbf{A}_{j}\right) \underline{\psi}, \\
& \text { for every } j=l+1, l+2, \ldots, \kappa \text {, } \\
& \mathbf{A} \underline{\mathbf{a}}=\underline{\mathbf{b}} \Longleftrightarrow \mathbf{A}\left(\mathbf{A}_{j}^{(1)} \underline{\mathbf{d}}_{j}\left(0^{+}\right)+\left(I_{n}-\mathbf{A}_{j}^{(1)} \mathbf{A}_{j}\right) \underline{\psi}\right)=\underline{\mathbf{b}} \\
& \Leftrightarrow \mathbf{A A}_{j}^{(1)} \underline{\mathbf{d}}_{j}\left(0^{+}\right)+\mathbf{A}\left(I_{n}-\mathbf{A}_{j}^{(1)} \mathbf{A}_{j}\right) \underline{\psi}=\underline{\mathbf{b}} \\
& \Leftrightarrow\left[\begin{array}{c}
\mathbf{V}_{l} \\
\mathbf{A}_{l+1} \\
\mathbf{A}_{l+2} \\
\vdots \\
\mathbf{A}_{\kappa}
\end{array}\right] \mathbf{A}_{j}^{(1)} \underline{\mathbf{d}}_{j}\left(0^{+}\right)+\left(\left[\begin{array}{c}
\mathbf{V}_{l} \\
\mathbf{A}_{l+1} \\
\mathbf{A}_{l+2} \\
\vdots \\
\mathbf{A}_{\kappa}
\end{array}\right]-\left[\begin{array}{c}
\mathbf{V}_{l} \\
\mathbf{A}_{l+1} \\
\mathbf{A}_{l+2} \\
\vdots \\
\mathbf{A}_{\kappa}
\end{array}\right] \mathbf{A}_{j}^{(1)} \mathbf{A}_{j}\right) \underline{\psi} \\
& \triangleq\left[\begin{array}{c}
\underline{\mathbf{z}}_{l}^{*}\left(0^{+}\right) \\
\underline{\mathbf{d}}_{l+1}\left(0^{+}\right) \\
\underline{\mathbf{d}}_{l+2}\left(0^{+}\right) \\
\vdots \\
\underline{\mathbf{d}}_{\kappa}\left(0^{+}\right)
\end{array}\right] \Longleftrightarrow\left[\begin{array}{c}
\mathbf{V}_{l} \mathbf{A}_{j}^{(1)} \underline{\mathbf{d}}_{j}\left(0^{+}\right) \\
\mathbf{A}_{l+1} \mathbf{A}_{j}^{(1)} \underline{\mathbf{d}}_{j}\left(0^{+}\right) \\
\mathbf{A}_{l+2} \mathbf{A}_{j}^{(1)} \underline{\mathbf{d}}_{j}\left(0^{+}\right) \\
\vdots \\
\mathbf{A}_{\kappa} \mathbf{A}_{j}^{(1)} \underline{\mathbf{d}}_{j}\left(0^{+}\right)
\end{array}\right] \\
& +\left[\begin{array}{c}
\mathbf{V}_{l}\left(I_{n}-\mathbf{A}_{j}^{(1)} \mathbf{A}_{j}\right) \\
\mathbf{A}_{l+1}\left(I_{n}-\mathbf{A}_{j}^{(1)} \mathbf{A}_{j}\right) \\
\mathbf{A}_{l+2}\left(I_{n}-\mathbf{A}_{j}^{(1)} \mathbf{A}_{j}\right) \\
\vdots \\
\mathbf{A}_{\kappa}\left(I_{n}-\mathbf{A}_{j}^{(1)} \mathbf{A}_{j}\right)
\end{array}\right] \underline{\psi}=\left[\begin{array}{c}
\underline{\mathbf{z}}_{l}^{*}\left(0^{+}\right) \\
\underline{\mathbf{d}}_{l+1}\left(0^{+}\right) \\
\underline{\mathbf{d}}_{l+2}\left(0^{+}\right) \\
\vdots \\
\underline{\mathbf{d}}_{\kappa}\left(0^{+}\right)
\end{array}\right] .
\end{aligned}
$$

From the above equalities, the expressions (122)-(124) are obtained.

With this important theorem, the discussion of system (32) has finished. In the next section, we present the solution of system (24).

\section{The Solution of Subsystem (24)}

The differential system of (15) is given by

$$
H_{q} \underline{y}_{p}^{(r)}(t)=\underline{y}_{q}(t)+\underline{\tilde{b}}_{q} u_{o}(t) .
$$

We start this subsection by observing that-as is well known, see Section 2-there exists a $q^{*} \in \mathbb{N}$ such that $H_{q}^{q^{*}}=\mathbb{O}$, that is, $q^{*}$ is the annihilation index of $H_{q}$. We obtain

$$
\begin{aligned}
H_{q} \underline{y}_{p}^{(r)}(t) & =\underline{y}_{q}(t)+\underline{\tilde{b}}_{q} u_{o}(t) \Longrightarrow H_{q}^{q^{*}} \underline{y}_{p}^{(r)}(t) \\
& =H_{q}^{q^{*}-1} \underline{y}_{q}(t)+H_{q}^{q^{*}-1} \underline{\tilde{b}}_{q} u_{o}(t)
\end{aligned}
$$


or equivalently

$$
H_{q}^{q^{*}-1} \underline{y}_{q}(t)+H_{q}^{q^{*}-1} \underline{\tilde{b}}_{q} u_{o}(t)=\underline{0}_{q} .
$$

Afterwards, we present a condition, in order to accept the already known solution $\underline{\mathbf{a}}$ (see Section 3 ).

We know that the nilpotent block matrix $H_{q}$ is given by

$$
H_{q_{1}} \bigoplus H_{q_{2}} \bigoplus \cdots \oplus H_{q_{\mu}}
$$

Now, taking into consideration that $H_{q}^{q^{*}}=\mathbb{O}$, it follows that for each

$$
\begin{gathered}
H_{q^{*}}^{q^{*}-1}=\left[\begin{array}{cccc}
0 & \cdots & 0 & 1 \\
0 & \cdots & 0 & 0 \\
\vdots & \cdots & \vdots & \vdots \\
0 & \cdots & 0 & 0
\end{array}\right], \\
q^{*}=\max \left\{q_{j}: j=1,2, \ldots, \mu\right\} .
\end{gathered}
$$

Lemma 21 (see [5]). All the elements of the block diagonal matrix $H_{q}^{q^{*}-1}$ are zero, that is, $H_{i j}^{q^{*}-1}=0$ apart from the $\xi$ elements at positions $\left(1, q^{*}\right),\left(q^{*}+1,2 q^{*}\right), \ldots,\left((\xi-1) q^{*}+\right.$ $\left.1, \xi q^{*}\right)$ which are one. Note that $\xi$ is the number of maximum block matrices with the same dimension, that is, $q^{*} \times q^{*}$, where $q^{*}=\max \left\{q_{j}: j=1,2, \ldots, r\right\}$.

Theorem 22. The condition which makes the system (24) solvable and provides an acceptable choice of vector $\underline{\mathbf{a}}$ is given by

$$
H_{q}^{q^{*}-1} \underline{y}_{q}\left(0^{+}\right)=\underline{0}_{q}
$$

Proof. First, we obtain system (129), that is, $H_{q}^{q^{*}-1} \underline{y}_{q}(t)+$ $H_{q}^{q^{*}-1} \tilde{b}_{q} u_{o}(t)=\underline{0}_{q}$. Then, for the solution of system (129), we take into account that

$$
\begin{aligned}
u_{0}(t)=\lim _{\sigma \rightarrow 0}( & \alpha_{0} \frac{1}{\sigma} \Phi\left(\frac{t}{\sigma}\right)+\alpha_{1} \frac{1}{\sigma^{2}} \Phi^{\prime}\left(\frac{t}{\sigma}\right) \\
& \left.+\cdots+\alpha_{n-1} \frac{1}{\sigma^{n}} \Phi^{(n-1)}\left(\frac{t}{\sigma}\right)\right) .
\end{aligned}
$$

Following Remark 1, we can assume that $t=K \sigma$ with $K$ big enough so that

$$
\Phi^{(k)}(K) \longrightarrow 0, \quad \text { for every } k=1,2, \ldots, n .
$$

Moreover, we have $K \sigma \rightarrow 0^{+}$and $t \rightarrow 0^{+}$. Then, we take

$$
\begin{aligned}
\lim _{t \rightarrow 0^{+}}\left(H_{q}^{q^{*}-1} \underline{y}_{q}(t)+H_{q}^{q^{*}-1} \underline{\tilde{b}}_{q} u_{o}(t)\right) \\
\quad=\underline{0}_{q} \Longleftrightarrow H_{q}^{q^{*}-1} \lim _{t \rightarrow 0^{+}} \underline{y}_{q}(t)+H_{q}^{q^{*}-1} \tilde{\underline{b}}_{q} \lim _{\sigma \rightarrow 0} u(\mathrm{t}) .
\end{aligned}
$$

However,

$$
\begin{aligned}
& \lim _{\sigma \rightarrow 0} u(t)=\lim _{\sigma \rightarrow 0}\left(\alpha_{0} \frac{1}{\sigma} \Phi\left(\frac{t}{\sigma}\right)+\cdots+\alpha_{n-1} \frac{1}{\sigma^{n}} \Phi^{(n-1)}\left(\frac{t}{\sigma}\right)\right) \\
&= \lim _{\sigma \rightarrow 0}\left(\alpha_{0} \frac{1}{\sigma} \Phi(K)+\cdots+\frac{1}{\sigma^{n}} \Phi^{(n-1)}(K)\right)=0, \\
& \lim _{t \rightarrow 0^{+}} \underline{y}_{q}(t)=\underline{y}_{q}\left(0^{+}\right) .
\end{aligned}
$$

Thus, (132) is derived.

An equivalent proof to [5] for Theorem 23 is provided.

Theorem 23. A necessary condition for obtaining the solution of system (24) is given by

$$
\underline{x}\left(0^{+}\right)=\left(I_{n}-G^{(1)} G\right) X,
$$

where

$$
\begin{aligned}
G & =\left[\begin{array}{cccc}
\tilde{q}_{p+q^{*}, 1} & \tilde{q}_{p+q^{*}, 1} & \cdots & \tilde{q}_{p+q^{*}, n} \\
\tilde{q}_{p+2 q^{*}, 1} & \tilde{q}_{p+2 q^{*}, 2} & \cdots & \tilde{q}_{p+2 q^{*}, n} \\
\vdots & \vdots & \cdots & \vdots \\
\tilde{q}_{p+\xi q^{*}, 1} & \tilde{q}_{p+\xi q^{*}, 2} & \cdots & \tilde{q}_{p+\xi q^{*}, n}
\end{array}\right] \\
& \in \mathcal{M}_{\xi n} ;\left(\tilde{q}_{i j}\right)_{\substack{i=p+q^{*}, \ldots, p+r q^{*} \\
j=1,2, \ldots, n}}
\end{aligned}
$$

are elements of the matrix $\widetilde{Q}$, the $G^{(1)}$ is $\{1\}$-inverse of matrix $G$, and for an arbitrarily chosen $X \in \mathcal{M}_{n 1}$.

Proof. Considering the transformation (25), we obtain

$$
\underline{y}\left(0^{+}\right)=Q^{-1} \underline{x}\left(0^{+}\right)
$$

or equivalently by defining $\widetilde{Q} \triangleq Q^{-1}$

$$
\begin{aligned}
{\left[\begin{array}{l}
\underline{y}_{p}\left(0^{+}\right) \\
\underline{y}_{q}\left(0^{+}\right)
\end{array}\right] } & =\left(\tilde{q}_{i j}\right)_{i, j=1,2, \ldots, n}\left[\begin{array}{l}
\underline{x}_{p}\left(0^{+}\right) \\
\underline{x}_{q}\left(0^{+}\right)
\end{array}\right] \Longleftrightarrow\left[\begin{array}{c}
\underline{y}_{p}\left(0^{+}\right) \\
\underline{y}_{q}\left(0^{+}\right)
\end{array}\right] \\
& =\left(\sum_{j=1}^{n} \tilde{q}_{i j} x_{j}\left(0^{+}\right)\right)_{i=1,2, \ldots, n}
\end{aligned}
$$

and finally

$$
H_{q}^{q^{*}-1} \underline{y}_{q}\left(0^{+}\right)=\underline{0}_{q} \Longrightarrow\left\{\begin{array}{l}
\sum_{j=1}^{n} \tilde{q}_{p+q^{*}, j} x_{j}\left(0^{+}\right)=0, \\
\sum_{j=1}^{n} \tilde{q}_{p+2 q^{*}, j} x_{j}\left(0^{+}\right)=0, \\
\vdots \\
\sum_{j=1}^{n} \tilde{q}_{p+\xi q^{*}, j} x_{j}\left(0^{+}\right)=0 .
\end{array}\right.
$$

System (141) can be expressed as follows:

$$
G \underline{x}\left(0^{+}\right)=\underline{0},
$$


where

$$
G=\left[\begin{array}{cccc}
\tilde{q}_{p+q^{*}, 1} & \tilde{q}_{p+q^{*}, 1} & \cdots & \tilde{q}_{p+q^{*}, n} \\
\tilde{q}_{p+2 q^{*}, 1} & \tilde{q}_{p+2 q^{*}, 2} & \cdots & \tilde{q}_{p+2 q^{*}, n} \\
\vdots & \vdots & \cdots & \vdots \\
\tilde{q}_{p+\xi q^{*}, 1} & \tilde{q}_{p+\xi q^{*}, 2} & \cdots & \tilde{q}_{p+\xi q^{*}, n}
\end{array}\right] \in \mathcal{M}_{\xi n} .
$$

The proof is concluded by using the results of Theorem 22 .

\section{An Illustrative Numerical Application}

In this section, a numerical example of the above method is presented. We consider the second-order system

$$
F \underline{x}^{\prime \prime}(t)=G \underline{x}(t)+\underline{b} u(t)
$$

where the matrix coefficients are given by

$$
\begin{aligned}
F & =\left[\begin{array}{ccccccc}
0 & 1 & -1 & 0 & 1 & 1 & 0 \\
1 & -6 & 3 & -2 & 1 & -1 & -2 \\
1 & -1 & 1 & -1 & 0 & -1 & -1 \\
1 & -3 & 2 & -1 & 0 & -1 & -1 \\
0 & 0 & 0 & 0 & 0 & 0 & 0 \\
0 & 0 & 1 & 0 & -1 & -1 & 0 \\
0 & -3 & 1 & -1 & 1 & 0 & 0
\end{array}\right], \\
G & =\left[\begin{array}{ccccccc}
1 & 0 & 1 & -1 & 0 & -1 & -1 \\
-2 & -2 & -2 & 1 & 1 & 3 & 4 \\
-1 & 2 & 0 & 1 & -2 & -1 & 1 \\
-1 & 0 & -1 & 1 & 0 & 1 & 2 \\
0 & 0 & 1 & 0 & -1 & -1 & 0 \\
-1 & 1 & -1 & 1 & 0 & 1 & 1 \\
-2 & -1 & -2 & 1 & 1 & 2 & 3
\end{array}\right],
\end{aligned}
$$

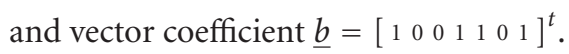

Let us suppose that the system at time $0^{-}$is

$$
\underline{x}\left(0^{-}\right)=\underline{x}^{\prime}\left(0^{-}\right)=\left[\begin{array}{lllllll}
0 & 0 & 0 & 0 & 0 & 0 & 0
\end{array}\right]^{t},
$$

and at time $0^{+}$it achieves

$$
\underline{x}\left(0^{+}\right)=\underline{x}^{\prime}\left(0^{+}\right)=\left[\begin{array}{lllllll}
-3 & -2 & 2 & 3 & 1 & 2 & 1
\end{array}\right]^{t} .
$$

From the regularity of $s F-G$, there exist nonsingular matrices in $\mathbb{R}^{7 \times 7}$

$$
P=\left[\begin{array}{ccccccc}
0 & 0 & 0 & 1 & 0 & 0 & -1 \\
1 & 0 & 0 & 0 & 0 & 1 & 0 \\
0 & -1 & 0 & 1 & 0 & 0 & 1 \\
1 & 0 & 0 & 1 & 0 & 0 & 0 \\
0 & 0 & -1 & 0 & 1 & 0 & 0 \\
0 & 0 & 0 & 0 & 0 & 1 & 0 \\
0 & 0 & 0 & 0 & 1 & 0 & 0
\end{array}\right],
$$

$$
Q=\left[\begin{array}{ccccccc}
1 & 0 & 0 & 1 & 0 & 0 & -1 \\
0 & 1 & 0 & 0 & 0 & 0 & 0 \\
0 & 1 & 0 & 0 & 1 & 1 & 1 \\
1 & 0 & 1 & 0 & 1 & 1 & 0 \\
0 & 1 & 1 & 0 & 1 & 0 & 0 \\
0 & 0 & -1 & 0 & 0 & 1 & 0 \\
0 & 0 & 0 & 1 & 0 & 0 & 0
\end{array}\right],
$$

such that

$$
\begin{gathered}
P F Q=F_{w}=\left[\begin{array}{cc}
I_{2} & \mathbb{O}_{5,2} \\
\mathbb{O}_{5,2} & H_{5}
\end{array}\right], \\
P G Q=G_{w}=\left[\begin{array}{cc}
J_{2}(1) & \mathbb{O}_{5,2} \\
\mathbb{O}_{5,2} & I_{5}
\end{array}\right],
\end{gathered}
$$

where $I_{2}, J_{2}(1), H_{5}$ are given by (17).

Consequently, system (144) is divided into the following subsystems:

$$
\underline{y}_{2}^{\prime \prime}(t)=\left[\begin{array}{ll}
2 & 1 \\
0 & 2
\end{array}\right] \underline{y}_{2}(t)+\underline{b}_{2} u_{0}(t),
$$

$$
\left[\begin{array}{lllll}
0 & 1 & 0 & & \\
0 & 0 & 1 & \\
0 & 0 & 0 & \\
& & & 0 & 1 \\
& & & 0 & 0
\end{array}\right] \underline{y}_{5}^{\prime \prime}(t)=\underline{y}_{5}(t)+\underline{b}_{5} u_{o}(t)
$$

Moreover, we obtain

$$
u_{o}(t)=a_{o} \delta(t)+a_{1} \delta^{(1)}(t)+\cdots+a_{5} \delta^{(5)}(t)+a_{6} \delta^{(6)}(t) .
$$


Before we go further with the analytic determination of the unknown coefficients $a_{i}$, we should verify the important condition (132). First, we calculate the inverse matrix of $Q$,

$$
Q^{-1}=\left(\widetilde{q}_{i j}\right)_{i, j=1,2, \ldots, n}=\left[\begin{array}{ccccccc}
1 & 0 & 1 & 0 & -1 & -1 & -1 \\
0 & 1 & 0 & 0 & 0 & 0 & 0 \\
-1 & 1 & -1 & 1 & 0 & 0 & 1 \\
0 & 0 & 0 & 0 & 0 & 0 & 1 \\
1 & -2 & 1 & -1 & 1 & 0 & -1 \\
-1 & 1 & -1 & 1 & 0 & 1 & 1 \\
0 & 0 & 1 & 0 & -1 & -1 & 0
\end{array}\right],
$$

$\underline{y}\left(0^{+}\right)=Q^{-1} \underline{x}\left(0^{+}\right)=\left[\begin{array}{l}\underline{y}_{2}\left(0^{+}\right) \\ \underline{y}_{5}\left(0^{+}\right)\end{array}\right]=\left[\begin{array}{lllllll}-5 & -2 & -3 & 1 & 0 & 5 & -1\end{array}\right]^{t}$.

Consequently, it is not difficult to verify that condition (132) holds, that is, $H_{5}^{2} y_{5}\left(0^{+}\right)=\underline{0}_{5}$.

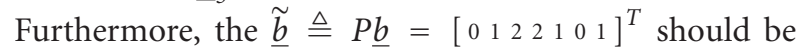
calculated. Thus

$$
\underline{\tilde{b}}_{2}=\left[\begin{array}{l}
0 \\
1
\end{array}\right], \quad \underline{\tilde{b}}_{5}=\left[\begin{array}{l}
2 \\
2 \\
1 \\
0 \\
1
\end{array}\right] .
$$

Now, the approximate expression for (151) is given by

$$
\begin{aligned}
u(t)= & \frac{a_{o}}{\sigma} \Phi\left(\frac{t}{\sigma}\right)+\frac{a_{1}}{\sigma^{2}} \Phi^{(1)}\left(\frac{t}{\sigma}\right) \\
& +\cdots+\frac{a_{5}}{\sigma^{5}} \Phi^{(5)}\left(\frac{t}{\sigma}\right)+\frac{a_{6}}{\sigma^{6}} \Phi^{(6)}\left(\frac{t}{\sigma}\right) .
\end{aligned}
$$

System (150) is a standard form of nonhomogeneous secondorder linear differential equations of Apostol-Kolodner type. Thus, using the results of Section 3.1, we obtain

$$
\underline{\mathbf{z}}^{\prime}(t)=\mathbf{R} \underline{\mathbf{z}}(t)+\underline{\mathbf{L}} u_{o}(t),
$$

where $\underline{\mathbf{z}}(t)=\left[\underline{z}_{1}^{t}(t) \underline{z}_{2}^{t}(t)\right]^{t}$ and the coefficient matrices $\mathbf{R}$ and $\underline{\mathbf{L}}$ are given by

$$
\begin{gathered}
\mathbf{R}=\left[\begin{array}{cc}
\mathbb{O}_{2} & I_{2} \\
J_{2}(1) & \mathbb{O}_{2}
\end{array}\right]=\left[\begin{array}{llll}
0 & 0 & 1 & 0 \\
0 & 0 & 0 & 1 \\
1 & 1 & 0 & 0 \\
0 & 1 & 0 & 0
\end{array}\right], \\
\underline{\mathbf{L}}=\left[\begin{array}{l}
\underline{0}_{2} \\
\tilde{\underline{b}}_{2}
\end{array}\right]=\left[\begin{array}{l}
0 \\
0 \\
0 \\
1
\end{array}\right] .
\end{gathered}
$$

Considering (25), at time $0^{+}$we obtain

$$
\underline{y}\left(0^{+}\right)=Q^{-1} \underline{x}\left(0^{+}\right)=\underline{y}^{\prime}\left(0^{+}\right)=Q^{-1} \underline{x}^{\prime}\left(0^{+}\right) .
$$

Moreover,

$$
\underline{y}_{2}\left(0^{+}\right)=\left[\begin{array}{l}
-5 \\
-2
\end{array}\right], \quad \underline{y}_{2}^{\prime}\left(0^{+}\right)=\left[\begin{array}{l}
-5 \\
-2
\end{array}\right] .
$$

Furthermore,

$$
\underline{\mathbf{z}}\left(0^{+}\right)=\left[\begin{array}{ll}
\underline{y}_{2}^{t}\left(0^{+}\right) & \underline{y}_{2}^{\prime t}\left(0^{+}\right)
\end{array}\right]^{t}=\left[\begin{array}{c}
-5 \\
-2 \\
-5 \\
-2
\end{array}\right] .
$$

There exists an invertible matrix

$$
C=\left[\begin{array}{cccc}
1 / 4 & 1 / 4 & 1 / 4 & -1 / 4 \\
0 & -1 / 2 & 0 & 1 / 2 \\
-1 / 4 & 0 & 1 / 4 & 0 \\
0 & 1 / 2 & 0 & 1 / 2
\end{array}\right]
$$

such that $\mathbf{J}=C^{-1} \mathbf{R} C$ is Jordan canonical form of matrix $\mathbf{R}$. Analytically,

$$
\mathbf{J}=\left[\begin{array}{cc}
-1 & 1 \\
0 & -1
\end{array}\right] \bigoplus\left[\begin{array}{ll}
1 & 1 \\
0 & 1
\end{array}\right]=J_{2}(-1) \bigoplus J_{2}(1)
$$

Now, taking into consideration (52) and Remark 1 the solution is given by

$$
C^{-1} \underline{\mathbf{z}}(t)=\left[\begin{array}{c}
\lim _{\sigma \rightarrow 0}\left\{\int_{-\infty}^{t} e^{-J_{2}(-1) \tau} \underline{\mathbf{b}}_{2} u(\tau) d \tau\right\} \\
\lim _{\sigma \rightarrow 0}\left\{\int_{-\infty}^{t} e^{-J_{2}(1) \tau} \underline{\mathbf{b}}_{2} u(\tau) d \tau\right\}
\end{array}\right],
$$

where

$$
\underline{\mathbf{b}} \triangleq C^{-1} \underline{\mathbf{L}}=\left[\begin{array}{l}
\underline{\mathbf{b}}_{2} \\
\underline{\mathbf{b}}_{2}
\end{array}\right]=\left[\begin{array}{l}
0 \\
1 \\
0 \\
1
\end{array}\right] \text {. }
$$


Using (55), we obtain

$$
C^{-1} \underline{\mathbf{z}}(t)=\left[\begin{array}{c}
-\int_{-\infty}^{t} \tau e^{\tau} u(\tau) d \tau \\
\int_{-\infty}^{t} e^{\tau} u(\tau) d \tau \\
-\int_{-\infty}^{t} e^{-\tau} \tau u(\tau) d \tau \\
\int_{-\infty}^{t} e^{-\tau} u(\tau) d \tau
\end{array}\right]=\Phi^{-1}(K)\left[\begin{array}{c}
\sum_{k=0}^{6} \alpha_{k} \frac{d \lambda_{1}^{k}}{d \lambda_{1}} \\
\sum_{k=0}^{6} \alpha_{k} \lambda_{1}^{k} \\
\sum_{k=0}^{6} \alpha_{k} \frac{d \lambda_{2}^{k}}{d \lambda_{2}} \\
\sum_{k=0}^{6} \alpha_{k} \lambda_{2}^{k}
\end{array}\right]
$$$$
=\Phi^{-1}(K)\left[\begin{array}{ccccccc}
0 & 1 & -2 & 3 & -4 & 5 & -6 \\
1 & -1 & 1 & -1 & 1 & -1 & 1 \\
0 & 1 & 2 & 3 & 4 & 5 & 6 \\
1 & 1 & 1 & 1 & 1 & 1 & 1
\end{array}\right] \underline{\mathbf{a}},
$$$$
\mathbf{A}=\left[\begin{array}{l}
\mathbf{A}_{\tau_{1}=2} \\
\mathbf{A}_{\tau_{2}=2}
\end{array}\right]=\left[\begin{array}{ccccccc}
1 & -1 & 1 & -1 & 1 & -1 & 1 \\
0 & 1 & -2 & 3 & -4 & 5 & -6 \\
1 & 1 & 1 & 1 & 1 & 1 & 1 \\
0 & 1 & 2 & 3 & 4 & 5 & 6
\end{array}\right]
$$

or equivalently

$$
\begin{gathered}
\mathbf{A}_{\tau_{1}=2}=\left[\begin{array}{ccccccc}
1 & -1 & 1 & -1 & 1 & -1 & 1 \\
0 & 1 & -2 & 3 & -4 & 5 & -6
\end{array}\right], \\
\mathbf{A}_{\tau_{2}=2}=\left[\begin{array}{ccccccc}
1 & 1 & 1 & 1 & 1 & 1 & 1 \\
0 & 1 & 2 & 3 & 4 & 5 & 6
\end{array}\right] .
\end{gathered}
$$

Thus,

$$
\begin{aligned}
P_{1}(2,1) \mathbf{A}_{\tau_{1}=2}= & {\left[\begin{array}{ccccccc}
1 & 0 & -1 & 2 & -3 & 4 & -5 \\
0 & 1 & -2 & 3 & -4 & 5 & -6
\end{array}\right]=\widetilde{\mathbf{A}}_{\tau_{1}=2}, } \\
\widetilde{\mathbf{A}}_{\tau_{1}=2} Q_{1} & (3,1) Q_{2}(3,2) Q_{1}(4,-2) Q_{2}(4,-3) Q_{1}(5,3) \\
& \times Q_{2}(5,4) Q_{1}(6,-4) Q_{2}(6,-5) Q_{1}(7,5) Q_{2}(7,6) \\
= & {\left[\begin{array}{lllllll}
1 & 0 & 0 & 0 & 0 & 0 & 0 \\
0 & 1 & 0 & 0 & 0 & 0 & 0
\end{array}\right], }
\end{aligned}
$$

where

$$
\mathbf{P}_{1}=\left[\begin{array}{ll}
1 & 1 \\
0 & 1
\end{array}\right], \quad \mathbf{Q}_{1}=\left[\begin{array}{ccccccc}
1 & 0 & 1 & -2 & 3 & -4 & 5 \\
0 & 1 & 2 & -3 & 4 & -5 & 6 \\
0 & 0 & 1 & 0 & 0 & 0 & 0 \\
0 & 0 & 0 & 1 & 0 & 0 & 0 \\
0 & 0 & 0 & 0 & 1 & 0 & 0 \\
0 & 0 & 0 & 0 & 0 & 1 & 0 \\
0 & 0 & 0 & 0 & 0 & 0 & 1
\end{array}\right] .
$$

Moreover,

$$
\begin{aligned}
& P_{1}(2,-1) \mathbf{A}_{\tau_{2}=2}=\left[\begin{array}{ccccccc}
1 & 0 & -1 & -2 & -3 & -4 & -5 \\
0 & 1 & 2 & 3 & 4 & 5 & 6
\end{array}\right]=\widetilde{\mathbf{A}}_{\tau_{2}=2}, \\
& \widetilde{\mathbf{A}}_{\tau_{1}=2} Q_{1}(3,1) Q_{2}(3,2) Q_{1}(4,2) Q_{2}(4,-3) Q_{1}(5,3) \\
& \times Q_{2}(5,-4) Q_{1}(6,4) Q_{2}(6,-5) Q_{1}(7,5) Q_{2}(7,-6) \\
& =\left[\begin{array}{lllllll}
1 & 0 & 0 & 0 & 0 & 0 & 0 \\
0 & 1 & 0 & 0 & 0 & 0 & 0
\end{array}\right]
\end{aligned}
$$

where

$$
\begin{gathered}
\mathbf{P}_{2}=\left[\begin{array}{cc}
1 & -1 \\
0 & 1
\end{array}\right], \\
\mathbf{Q}_{2}=\left[\begin{array}{ccccccc}
1 & 0 & 1 & 2 & 3 & 4 & 5 \\
0 & 1 & -2 & -3 & -4 & -5 & -6 \\
0 & 0 & 1 & 0 & 0 & 0 & 0 \\
0 & 0 & 0 & 1 & 0 & 0 & 0 \\
0 & 0 & 0 & 0 & 1 & 0 & 0 \\
0 & 0 & 0 & 0 & 0 & 1 & 0 \\
0 & 0 & 0 & 0 & 0 & 0 & 1
\end{array}\right] .
\end{gathered}
$$

Thus, considering the result of Section 3 , the $\{1\}$-inverse of matrices $\mathbf{A}_{\tau_{1}=2}$ and $\mathbf{A}_{\tau_{2}=2}$ are given by,

$$
Q_{1}\left[\begin{array}{c}
I_{2} \\
\mathbb{O}_{5,2}
\end{array}\right] \mathbf{P}_{1}, \quad \mathbf{Q}_{2}\left[\begin{array}{c}
I_{2} \\
\mathbb{O}_{5,2}
\end{array}\right] \mathbf{P}_{2} \text {, respectively, }
$$

Finally, the general solution is given by the expression

$$
\underline{\mathbf{a}}=\frac{1}{\Phi^{-1}(K)} \mathbf{A}_{\tau_{1}=2}^{(1)} \underline{\mathbf{d}}_{\tau_{1}=2}\left(0^{+}\right)+\left(I_{7}-\mathbf{A}_{\tau_{1}=2}^{(1)} \mathbf{A}_{\tau_{1}=2}\right) \underline{\psi}
$$

where the following conditions should also be satisfied:

$$
\begin{aligned}
& \mathbf{A}_{\tau_{1}=2}\left[\frac{1}{\Phi^{-1}(K)} \mathbf{A}_{\tau_{2}=2}^{(1)} \underline{\mathbf{d}}_{\tau_{2}=2}\left(0^{+}\right)+\left(I_{7}-\mathbf{A}_{\tau_{2}=2}^{(1)} \mathbf{A}_{\tau_{2}=2}\right) \underline{\psi}\right]=\underline{\mathbf{d}}_{\tau_{1}=2}, \\
& \mathbf{A}_{\tau_{2}=2}\left[\frac{1}{\Phi^{-1}(K)} \mathbf{A}_{\tau_{1}=2}^{(1)} \underline{\mathbf{d}}_{\tau_{1}=2}\left(0^{+}\right)+\left(I_{7}-\mathbf{A}_{\tau_{1}=2}^{(1)} \mathbf{A}_{\tau_{1}=2}\right) \underline{\psi}\right]=\underline{\mathbf{d}}_{\tau_{2}=2} .
\end{aligned}
$$


Analytically,

$$
\begin{gathered}
\underline{\mathbf{d}}\left(0^{+}\right)=C^{-1} \underline{\mathbf{z}}\left(0^{+}\right)=\left[\begin{array}{l}
\underline{\mathbf{d}}_{\tau_{1}=2}\left(0^{+}\right) \\
\underline{\mathbf{d}}_{\tau_{2}=2}\left(0^{+}\right)
\end{array}\right]=\frac{1}{\Phi^{-1}(K)}\left[\begin{array}{c}
-2 \\
0 \\
-22 \\
-4
\end{array}\right], \\
\underline{\mathbf{a}}=\frac{1}{\Phi^{-1}(K)}\left[\begin{array}{c}
-2 \\
0 \\
0 \\
0 \\
0 \\
0 \\
0
\end{array}\right]+\left[\begin{array}{lllllll}
0 & 0 & 1 & -2 & 3 & -4 & 5 \\
0 & 0 & 2 & -3 & 4 & -5 & 6 \\
& & 1 & & & \\
& & & 1 & & \\
& & & & 1 & \\
& & & & & 1
\end{array}\right]\left[\begin{array}{c}
\psi_{1} \\
\psi_{2} \\
\psi_{3} \\
\psi_{4} \\
\psi_{5} \\
\psi_{6} \\
\psi_{7}
\end{array}\right],
\end{gathered}
$$

where

$$
\begin{aligned}
& {\left[\begin{array}{ccccccc}
1 & -1 & 1 & -1 & 1 & -1 & 1 \\
0 & 1 & -2 & 3 & -4 & 5 & -6
\end{array}\right]} \\
& \left.\times \frac{1}{\Phi^{-1}(K)}\left[\begin{array}{c}
-2 \\
0 \\
0 \\
0 \\
0 \\
0 \\
0
\end{array}\right]+\left[\begin{array}{ccccccc}
0 & 0 & 1 & -2 & 3 & -4 & 5 \\
0 & 0 & 2 & -3 & 4 & -5 & 6 \\
& & 1 & & & & \\
& & & 1 & & & \\
& & & & 1 & & \\
& & & & & 1 & \\
\psi_{3} \\
\psi_{4} \\
\psi_{5} \\
\psi_{6} \\
\psi_{7}
\end{array}\right]\right]\left[\begin{array}{c}
\psi_{1} \\
\psi_{2} \\
\\
\end{array}\right. \\
& =\frac{1}{\Phi^{-1}(K)}\left[\begin{array}{c}
-2 \\
0
\end{array}\right] \\
& \Leftrightarrow \frac{1}{\Phi^{-1}(K)}\left[\begin{array}{c}
-2 \\
0
\end{array}\right]+\left[\begin{array}{c}
-\psi_{3}+\psi_{4}-\psi_{5}+\psi_{6}-\psi_{7} \\
2 \psi_{3}-3 \psi_{4}+4 \psi_{5}-5 \psi_{6}+6 \psi_{7}
\end{array}\right] \\
& =\frac{1}{\Phi^{-1}(K)}\left[\begin{array}{c}
-2 \\
0
\end{array}\right] \text {, }
\end{aligned}
$$$$
\left[\begin{array}{lllllll}
1 & 1 & 1 & 1 & 1 & 1 & 1 \\
0 & 1 & 2 & 3 & 4 & 5 & 6
\end{array}\right]
$$

$$
\left.\times \frac{1}{\Phi^{-1}(K)}\left[\begin{array}{c}
-18 \\
-4 \\
0 \\
0 \\
0 \\
0 \\
0
\end{array}\right]+\left[\begin{array}{ccccccc}
0 & 0 & 1 & 2 & 3 & 4 & 5 \\
0 & 0 & -2 & -3 & -4 & -5 & -6 \\
& & 1 & & & & \\
& & & 1 & & & \\
& & & & 1 & & \\
& & & & & & \\
\psi_{3} & \\
\psi_{4} \\
\psi_{5} \\
\psi_{6} \\
\psi_{7}
\end{array}\right]\right]\left[\begin{array}{c}
\psi_{1} \\
\psi_{2}
\end{array}\right]
$$

$$
\begin{aligned}
& =\frac{1}{\Phi^{-1}(K)}\left[\begin{array}{c}
-22 \\
-4
\end{array}\right] \\
& \Leftrightarrow \frac{1}{\Phi^{-1}(K)}\left[\begin{array}{c}
-22 \\
-4
\end{array}\right]-\left[\begin{array}{c}
\psi_{3}+\psi_{4}+\psi_{5}+\psi_{6}+\psi_{7} \\
2 \psi_{3}+3 \psi_{4}+4 \psi_{5}+5 \psi_{6}+6 \psi_{7}
\end{array}\right] \\
& =\frac{1}{\Phi^{-1}(K)}\left[\begin{array}{c}
-22 \\
-4
\end{array}\right] .
\end{aligned}
$$

Following the above conditions, we can see that $\psi_{4}=\psi_{6}=0$, $\psi_{3}, \psi_{5}$, and $\psi_{7}$ are arbitrary. Thus, we conclude this numerical example with the determination of the unknown coefficients $a_{i}$, for $i=0,1, \ldots, 6$

$$
\underline{\mathbf{a}}=\left[\begin{array}{c}
a_{0} \\
a_{1} \\
a_{2} \\
a_{3} \\
a_{4} \\
a_{5} \\
a_{6}
\end{array}\right]=\left[\begin{array}{c}
-\frac{2}{\Phi^{-1}(K)}+\psi_{3}+3 \psi_{5}+5 \psi_{7} \\
2 \psi_{3}+4 \psi_{5}+6 \psi_{7} \\
\psi_{3} \\
0 \\
\psi_{5} \\
0 \\
\psi_{7}
\end{array}\right]
$$

for arbitrary $\psi_{i} \in \mathbb{R}$, for $i=3,5,7$ and $\Phi^{-1}(\mathrm{~K})=$ $(1 / \sqrt{2 \pi}) \int_{-\infty}^{K} e^{-x^{2} / 2} d x ; K$ is large enough (i.e., $\left.K \rightarrow \infty\right)$ that the assumption $\lim _{x \rightarrow K}(1 / \sqrt{2 \pi}) e^{-x^{2} / 2}=0$ is valid.

\section{Conclusions}

In this paper, an analytical method and several sufficient conditions were proposed and discussed for the changing of the state of a higher-order linear descriptor (regular) differential system in (almost) zero time by using the impulse function and its derivatives. The input vector has to be made up a linear combination of the $\delta$-function of Dirac and its derivatives approximated using the normal (Gaussian) probability density function. Using the tools of linear algebra and generalized inverses, exact calculations of the relevant matrices are obtained.

To date, no other approximating function has been used in the analysis of the problem studied in this paper. It will be of interest to use some other approximations and obtain comparative results. In $[14,18,21]$, there are only some hints about the minimum time. However, there is no known formula which can calculate the minimum time considering some other significant problem parameters, such as the volatility $\sigma, K$ and so forth. 
Since we have applied an approximation for the impulse input, we transfer the initial state $\varepsilon$-close to a desired state. Consequently, a natural question is to ask how close we can get, and how we can calculate that distance, and if it is possible to minimize it.

\section{Appendices}

\section{A. The Analytic Calculation of Integral}

$$
\lim _{\sigma \rightarrow 0} \int_{-\infty}^{t} e^{-J_{0} t} \underline{b}_{s} u(\tau) d \tau
$$

Consider that

$$
\begin{aligned}
& \lim _{\sigma \rightarrow 0} \int_{-\infty}^{t} e^{-J_{0} t} \underline{\mathbf{b}}_{s} u(\tau) d \tau \\
& =\int_{-\infty}^{t}\left[\begin{array}{cccc}
e^{-J_{1} \tau} & & & \\
& \ddots & & \\
& & \ddots & \\
& & & e^{-J_{l} \tau}
\end{array}\right]\left[\begin{array}{c}
\underline{\mathbf{b}}_{\tau_{1}} \\
\underline{\mathbf{b}}_{\tau_{2}} \\
\vdots \\
\underline{\mathbf{b}}_{\tau_{l}}
\end{array}\right] u(\tau) d \tau,
\end{aligned}
$$

and finally, we obtain

$$
\lim _{\sigma \rightarrow 0} \int_{-\infty}^{t} e^{-J_{0} t} \underline{\mathbf{b}}_{s} u(\tau) d \tau=\left[\begin{array}{c}
\int_{-\infty}^{t} e^{-J_{1} \tau} \underline{\mathbf{b}}_{\tau_{1}} u(\tau) d \tau \\
\vdots \\
\int_{-\infty}^{t} e^{-J_{i} \tau} \underline{\mathbf{b}}_{\tau_{i}} u(\tau) d \tau \\
\vdots \\
\int_{-\infty}^{t} e^{-J_{l} \tau} \underline{\mathbf{b}}_{\tau_{l}} u(\tau) d \tau
\end{array}\right] \in \mathcal{M}_{s 1}
$$

with $i=1,2, \ldots, l$ and $\underline{\mathbf{b}}_{\tau_{i}} \in \mathcal{M}_{\left(\tau_{i}\right) 1}$. Moreover, we take for every $i=1,2, \ldots, l$,

$$
\begin{aligned}
\int_{-\infty}^{t} e^{-J_{i} \tau} \underline{\mathbf{b}}_{\tau_{i}} u(\tau) d \tau & =\int_{-\infty}^{t} e^{-\lambda_{i} \tau} \underline{\mathbf{b}}_{\tau_{i}} u(\tau) d \tau \\
& =\underline{\mathbf{b}}_{\tau_{i}} \int_{-\infty}^{t} e^{-\lambda_{i} \tau} u(\tau) d \tau .
\end{aligned}
$$

Afterwards, we calculate the integral $\int_{-\infty}^{t} e^{-\lambda_{i} \tau} \mathcal{u}(\tau) d \tau$. Analytically, considering also (21), we obtain

$$
\int_{-\infty}^{t} e^{-\lambda_{i} \tau} u(\tau) d \tau=\int_{-\infty}^{t} e^{-\lambda_{i} \tau} \sum_{k=0}^{n-1} \frac{1}{\sigma^{k+1}} \Phi^{(k)}\left(\frac{\tau}{\sigma}\right) a_{k} d \tau
$$

for every $i=1,2, \ldots, q$. Thus,

$$
\int_{-\infty}^{t} e^{-\lambda_{i} \tau} u(\tau) d \tau=\sum_{k=0}^{n-1} a_{k} \int_{-\infty}^{t} e^{-\lambda_{i} \tau} \frac{1}{\sigma^{k+1}} \Phi^{(k)}\left(\frac{\tau}{\sigma}\right) d \tau,
$$

where $(1 / \sigma) \Phi^{0}(\tau / \sigma)=(1 / \sigma) \Phi(\tau / \sigma)$ and $\Phi^{(m)}(\tau / \sigma)=d^{m} \Phi(\tau /$ $\sigma) / d(\tau / \sigma)^{m}$.

Remark 5. It is well-known that $\int_{-\infty}^{t}(1 / \sqrt{2 \pi}) e^{-\omega^{2} / 2} d \omega=$ $\Phi^{-1}(t)$. Consequently,

$$
\int_{-\infty}^{t} \Phi(\omega) d \omega=\Phi^{-1}(t)
$$

where $\Phi^{-1}(t)$ is the inverse function of normal probability distribution which is also a continuous one.

Lemma 24. One has

$$
\begin{aligned}
\int_{-\infty}^{t} e^{-\lambda \tau} \frac{1}{\sigma^{k+1}} \Phi^{(k)}\left(\frac{\tau}{\sigma}\right) d \tau \\
\quad=e^{-\lambda t}\left(\sum_{\ell=0}^{k-1} \lambda^{k-\ell-1} \frac{\Phi^{(\ell)}(t / \sigma)}{\sigma^{\ell+1}}\right)+\lambda^{k} e^{\lambda^{2} \sigma^{2} / 2} \Phi^{-1}\left(\frac{t}{\sigma}+\lambda \sigma\right) .
\end{aligned}
$$

Proof. First, we calculate the integral

$$
\begin{aligned}
& \int_{-\infty}^{t} e^{-\lambda \tau} \frac{1}{\sigma} \Phi\left(\frac{\tau}{\sigma}\right) d \tau \\
& \quad=e^{\left(\lambda^{2} \sigma^{2}\right) / 2} \cdot \frac{1}{\sigma} \int_{-\infty}^{t} \frac{1}{\sqrt{2 \pi}} e^{-(\tau / \sigma+\lambda \sigma)^{2} / 2} d \tau .
\end{aligned}
$$

Afterwards, we denote $(\tau / \sigma)+\lambda \sigma=\omega$. Then, $d \tau=\sigma \cdot d \omega$. Consequently, we obtain

$e^{\lambda^{2} \sigma^{2} / 2} \frac{1}{\sigma} \int_{-\infty}^{t / \sigma+\lambda \sigma} \frac{1}{\sqrt{2 \pi}} e^{-\omega^{2} / 2} \sigma d \omega=e^{\lambda^{2} \sigma^{2} / 2} \int_{-\infty}^{t / \sigma+\lambda \sigma} \frac{1}{\sqrt{2 \pi}} e^{-\omega^{2} / 2} d \omega$.

Then,

$$
\int_{-\infty}^{t} e^{-\lambda \tau} \frac{1}{\sigma} \Phi\left(\frac{\tau}{\sigma}\right) d \tau=e^{\lambda^{2} \sigma^{2} / 2} \Phi^{-1}\left(\frac{t}{\sigma}+\lambda \sigma\right) .
$$

Continuing our calculations, we take

$$
\begin{aligned}
& \int_{-\infty}^{t} e^{-\lambda \tau} \frac{\Phi^{(1)}(\tau / \sigma)}{\sigma^{2}} d \tau \\
& \quad=\frac{1}{\sigma}\left[e^{-\lambda \tau} \Phi\left(\frac{\tau}{\sigma}\right)\right]_{-\infty}^{t}+\lambda \frac{1}{\sigma} \int_{-\infty}^{t} e^{-\lambda \tau} \Phi\left(\frac{\tau}{\sigma}\right) d \tau \\
& \left(\text { But }_{\tau \rightarrow-\infty} e^{-\lambda \tau} \Phi\left(\frac{\tau}{\sigma}\right)=0\right) .
\end{aligned}
$$


Moreover, considering also (A.10), we obtain

$$
\begin{aligned}
\int_{-\infty}^{t} e^{-\lambda \tau} \frac{\Phi^{(1)}(\tau / \sigma)}{\sigma^{2}} d \tau & \\
& =\frac{1}{\sigma} e^{-\lambda t} \Phi\left(\frac{t}{\sigma}\right)+\lambda e^{\lambda^{2} \sigma^{2} / 2} \Phi^{-1}\left(\frac{t}{\sigma}+\lambda \sigma\right) .
\end{aligned}
$$

Continuing the above procedure, we have to calculate the integral $\int_{-\infty}^{t} e^{-\lambda \tau}\left(\Phi^{(2)}(\tau / \sigma) / \sigma^{3}\right) d \tau$. Thus,

$$
\int_{-\infty}^{t} e^{-\lambda \tau} \frac{\Phi^{(2)}(\tau / \sigma)}{\sigma^{3}} d \tau=
$$

(we denote $\tau / \sigma=z \Leftrightarrow \tau=\sigma z$, then $d \tau=\sigma d z$ and $-\infty / \sigma<$ $z<t / \sigma$. Note that $-\infty / \sigma=-\infty$ because $\sigma>0$-by definition of the normal probability distribution)

$$
\begin{aligned}
& =\int_{-\infty}^{t / \sigma} e^{-\lambda \sigma z} \frac{\Phi^{(2)}(z)}{\sigma^{3}} \cdot \sigma d z \\
& =\frac{1}{\sigma^{2}} e^{-\lambda t} \Phi^{(1)}\left(\frac{t}{\sigma}\right)+\frac{1}{\sigma^{2}} \lambda \sigma \int_{-\infty}^{t / \sigma} e^{-\lambda \sigma z} \Phi^{(1)}(z) d z=
\end{aligned}
$$

(we reuse the transformation $z=\tau / \sigma$ )

$$
\begin{aligned}
= & \frac{1}{\sigma^{2}} e^{-\lambda t} \Phi^{(1)}\left(\frac{t}{\sigma}\right) \\
& +\lambda\left[\frac{1}{\sigma} e^{-\lambda t} \Phi\left(\frac{t}{\sigma}\right)+\lambda e^{\left(\lambda^{2} \sigma^{2}\right) / 2} \Phi^{-1}\left(\frac{t}{\sigma}+\lambda \sigma\right)\right] .
\end{aligned}
$$

Consequently, we obtain

$$
\begin{aligned}
\int_{-\infty}^{t} e^{-\lambda \tau} \frac{\Phi^{(2)}(\tau / \sigma)}{\sigma^{3}} d \tau= & \frac{1}{\sigma^{2}} e^{-\lambda t} \Phi^{(1)}\left(\frac{t}{\sigma}\right)+\lambda \frac{1}{\sigma} e^{-\lambda t} \Phi\left(\frac{t}{\sigma}\right) \\
& +\lambda^{2} e^{\lambda^{2} \sigma^{2} / 2} \Phi^{-1}\left(\frac{t}{\sigma}+\lambda \sigma\right)
\end{aligned}
$$

Following the previous expressions, we assume that

$$
\begin{aligned}
& \int_{-\infty}^{t} e^{-\lambda \tau} \frac{1}{\sigma^{k+1}} \Phi^{(k)}\left(\frac{t}{\sigma}\right) d \tau \\
& \quad=e^{-\lambda t}\left(\sum_{\ell=0}^{k-1} \lambda^{k-\ell-1} \frac{\Phi^{(\ell)}(t / \sigma)}{\sigma^{\ell+1}}\right)+\lambda^{k} e^{\lambda^{2} \sigma^{2} / 2} \Phi^{-1}\left(\frac{t}{\sigma}+\lambda \sigma\right) .
\end{aligned}
$$

Now, in order to conclude the proof, the recursive formula should be proved, that is,

$$
\begin{aligned}
& \int_{-\infty}^{t} e^{-\lambda \tau} \frac{1}{\sigma^{k+2}} \Phi^{(k+1)}\left(\frac{\tau}{\sigma}\right) d \tau \\
& \quad=e^{-\lambda t}\left(\sum_{\ell=0}^{k} \lambda^{k-\ell} \frac{\Phi^{(\ell)}(t / \sigma)}{\sigma^{\ell+1}}\right)+\lambda^{k+1} e^{\lambda^{2} \sigma^{2} / 2} \Phi^{-1}\left(\frac{t}{\sigma}+\lambda \sigma\right) .
\end{aligned}
$$

Thus, we have

$$
\begin{aligned}
& \int_{-\infty}^{t} e^{-\lambda \tau} \frac{1}{\sigma^{k+2}} \Phi^{(k+1)}\left(\frac{\tau}{\sigma}\right) d \tau \quad\left(\text { denote } \frac{\tau}{\sigma}=z\right) \\
& =\frac{1}{\sigma^{k+1}} e^{-\lambda t} \Phi^{(k)}\left(\frac{t}{\sigma}\right)+\lambda \frac{1}{\sigma^{k+1}} \int_{-\infty}^{t / \sigma} e^{-\lambda \sigma z} \Phi^{(k)}(z) \sigma d z
\end{aligned}
$$

(reusing the transformation $z=\tau / \sigma$ )

$$
\begin{aligned}
& \stackrel{(\text { A.16) }}{=} \frac{1}{\sigma^{k+1}} e^{-\lambda t} \Phi^{(k)}\left(\frac{t}{\sigma}\right)+e^{-\lambda t} \sum_{\ell=0}^{k-1} \lambda^{k-\ell} \frac{\Phi^{(\ell)}(t / \sigma)}{\sigma^{\ell+1}} \\
& +\lambda^{k+1} e^{\lambda^{2} \sigma^{2} / 2} \Phi^{-1}\left(\frac{t}{\sigma}+\lambda \sigma\right) .
\end{aligned}
$$

However, the

$$
\begin{gathered}
\frac{1}{\sigma^{k+1}} e^{-\lambda t} \Phi^{(k)}\left(\frac{t}{\sigma}\right)+e^{-\lambda t} \sum_{\ell=0}^{k-1} \lambda^{k-\ell} \frac{\Phi^{(\ell)}(t / \sigma)}{\sigma^{\ell+1}} \\
=e^{-\lambda t} \sum_{\ell=0}^{k} \lambda^{k-\ell} \frac{\Phi^{(\ell)}(t / \sigma)}{\sigma^{\ell+1}} .
\end{gathered}
$$

Consequently, we have proved that

$$
\begin{aligned}
& \int_{-\infty}^{t} \sigma^{1 /(k+2)} e^{-\lambda \tau} \Phi^{(k+1)}\left(\frac{\tau}{\sigma}\right) d \tau \\
& =e^{-\lambda t}\left(\sum_{\ell=0}^{k} \lambda^{k-\ell} \frac{\Phi^{(\ell)}(t / \sigma)}{\sigma^{\ell+1}}\right)+\lambda^{k+1} e^{\lambda^{2} \sigma^{2} / 2} \Phi^{-1}\left(\frac{t}{\sigma}+\lambda \sigma\right) .
\end{aligned}
$$

And the proof has been concluded.

Lemma 25. One has

$$
\begin{aligned}
& \int_{-\infty}^{t} e^{-\lambda_{i} \tau} u(\tau) d \tau \\
&=\sum_{k=0}^{n-1} \alpha_{k}\left[e^{-\lambda_{i} t}\left(\sum_{\ell=0}^{k-1} \lambda_{i}^{k-\ell-1} \frac{\Phi^{(\ell)}(t / \sigma)}{\sigma^{\ell+1}}\right)\right. \\
&\left.+\lambda_{i}^{k} e^{\lambda_{i}^{2} \sigma^{2} / 2} \Phi^{-1}\left(\frac{t}{\sigma}+\lambda_{i} \sigma\right)\right] .
\end{aligned}
$$

Proof. We have seen that

$$
\begin{aligned}
& \int_{-\infty}^{t} e^{-\lambda_{i} \tau} u(\tau) d \tau \\
& \stackrel{(\mathrm{A} .17)}{=} \sum_{k=0}^{n-1} \alpha_{k}\left[e^{-\lambda_{i} t}\left(\sum_{\ell=0}^{k-1} \lambda_{i}^{k-\ell-1} \frac{\Phi^{(\ell)}(t / \sigma)}{\sigma^{\ell+1}}\right)\right. \\
&\left.+\lambda_{i}^{k} e^{\lambda_{i}^{2} \sigma^{2} / 2} \Phi^{-1}\left(\frac{t}{\sigma}+\lambda_{i} \sigma\right)\right] .
\end{aligned}
$$

Substituting (A.23) into (A.3), we obtain

$$
\begin{aligned}
& \underline{\mathbf{b}}_{\tau_{i}} \int_{-\infty}^{t} e^{-\lambda_{i} \tau} u(\tau) d \tau \\
&=\underline{\mathbf{b}}_{\tau_{i}} \sum_{k=0}^{n-1} \alpha_{k}\left[e^{-\lambda_{i} t}\left(\sum_{\ell=0}^{k-1} \lambda_{i}^{k-\ell-1} \frac{\Phi^{(\ell)}(t / \sigma)}{\sigma^{\ell+1}}\right)\right. \\
&\left.+\lambda_{i}^{k} e^{\lambda_{i}^{2} \sigma^{2} / 2} \Phi^{-1}\left(\frac{t}{\sigma}+\lambda_{i} \sigma\right)\right] .
\end{aligned}
$$


Thus, combining (A.2) and (A.25), we obtain

$$
\lim _{\sigma \rightarrow 0} \int_{-\infty}^{t} e^{-J_{0} t} \underline{\mathbf{b}}_{s} u(\tau) d \tau=\left[\begin{array}{c}
\underline{\mathbf{b}}_{\tau_{1}} \sum_{k=0}^{n-1} \alpha_{k}\left[e^{-\lambda_{1} t}\left(\sum_{\ell=0}^{k-1} \lambda_{1}^{k-\ell-1} \frac{\Phi^{(\ell)}(t / \sigma)}{\sigma^{\ell+1}}\right)+\lambda_{1}^{k} e^{\lambda_{1}^{2} \sigma^{2} / 2} \Phi^{-1}\left(\frac{t}{\sigma}+\lambda_{1} \sigma\right)\right] \\
\vdots \\
\underline{\mathbf{b}}_{\tau_{i}}^{n-1} \sum_{k=0}^{n}\left[e_{k}^{-\lambda_{i} t}\left(\sum_{\ell=0}^{k-1} \lambda_{i}^{k-\ell-1} \frac{\Phi^{(\ell)}(t / \sigma)}{\sigma^{\ell+1}}\right)+\lambda_{i}^{k} e^{\lambda_{i}^{2} \sigma^{2} / 2} \Phi^{-1}\left(\frac{t}{\sigma}+\lambda_{i} \sigma\right)\right] \\
\vdots \\
\underline{\mathbf{b}}_{\tau_{l}} \sum_{k=0}^{n-1} \alpha_{k}\left[e^{-\lambda_{l} t}\left(\sum_{\ell=0}^{k-1} \lambda_{l}^{k-\ell-1} \frac{\Phi^{(\ell)}(t / \sigma)}{\sigma^{\ell+1}}\right)+\lambda_{l}^{k} e^{\lambda_{l}^{2} \sigma^{2} / 2} \Phi^{-1}\left(\frac{t}{\sigma}+\lambda_{l} \sigma\right)\right]
\end{array}\right] \in \mathcal{M}_{s 1} .
$$

Lemma 26. One has

$$
\begin{array}{r}
\lim _{\sigma \rightarrow 0} \sum_{k=0}^{n-1} \alpha_{k}\left[e^{-\lambda_{i} t}\left(\sum_{\ell=0}^{k-1} \lambda_{i}^{k-\ell-1} \frac{\Phi^{(\ell)}(t / \sigma)}{\sigma^{\ell+1}}\right)\right. \\
\left.+\lambda_{i}^{k} e^{\lambda_{i}^{2} \sigma^{2} / 2} \Phi^{-1}\left(\frac{t}{\sigma}+\lambda_{i} \sigma\right)\right] \\
\approx \Phi^{-1}(K) \sum_{k=0}^{n-1} \underline{\alpha}_{k} \lambda_{i}^{k} \quad \text { for } i=1,2, \ldots, l .
\end{array}
$$

Proof. Considering here, Remark 2.1, and denoting $t=K \sigma$ with $K$ big enough such as

$$
\frac{\Phi^{(k)}(K)}{\sigma^{k+1}} \approx 0 \quad \text { for every } k=0,1,2, \ldots, n-1,
$$

then we obtain (A.27).

This appendix is finished with (A.29). This equation is a consequence of (A.26) and (A.27)

$$
\lim _{\sigma \rightarrow 0} \int_{-\infty}^{t} e^{-J_{0} t} \underline{\mathbf{b}}_{s} u(\tau) d \tau=\Phi^{-1}(K)\left[\begin{array}{c}
\underline{\mathbf{b}}_{\tau_{1}} \sum_{k=0}^{n-1} \alpha_{k} \lambda_{1}^{k} \\
\vdots \\
\underline{\mathbf{b}}_{\tau_{i}} \sum_{k=0}^{n-1} \alpha_{k} \lambda_{i}^{k} \\
\vdots \\
\underline{\mathbf{b}}_{\tau_{l}} \sum_{k=0}^{n-1} \alpha_{k} \lambda_{l}^{k}
\end{array}\right] \in \mathcal{M}_{s 1} .
$$

\section{B. The Analytic Calculation of Integral}

$\lim _{\sigma \rightarrow 0} \int_{-\infty}^{t} e^{-J_{i} t} \underline{b}_{\tau_{i}} u(\tau) d \tau$ for $i=l+1, l+2, \ldots, k$

Consider that

$$
\lim _{\sigma \rightarrow 0} \int_{-\infty}^{t} e^{-J_{j} t} \underline{\mathbf{b}}_{\tau_{j}} u(\tau) d \tau
$$

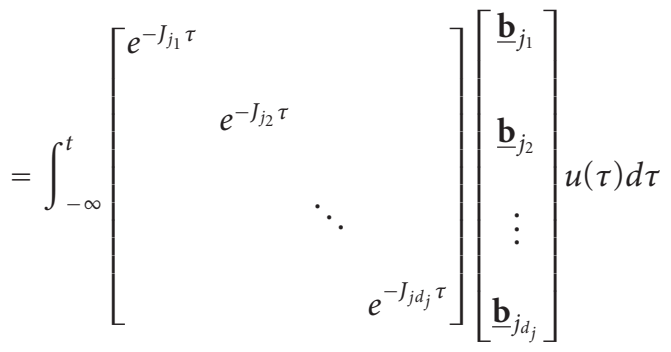

$$
=\left[\begin{array}{c}
\int_{-\infty}^{t} e^{-J_{j_{1}} \tau} \mathbf{T}_{j_{1}} u(\tau) d \tau \\
\int_{-\infty}^{t} e^{-J_{j 2} \tau} \mathbf{T}_{j_{2}} u(\tau) d \tau \\
\vdots \\
\int_{-\infty}^{t} e^{-J_{j_{j}} \tau} \mathbf{T}_{j_{d_{j}}} u(\tau) d \tau
\end{array}\right]
$$

$$
\text { with } z_{j}=1,2, \ldots, d_{j} \text { and the matrix } \underline{\mathbf{b}}_{z_{j}} \in \mathcal{M}_{\left(j_{z_{j}}\right) 1} \text {. }
$$


Thus,

$$
\begin{aligned}
& \left.\int_{-\infty}^{t} e^{-J_{z_{j}}{ }^{\tau}} \underline{\mathbf{b}}_{j_{j}} u(\tau) d \tau=\int_{-\infty}^{t}\left[\begin{array}{cccc}
e^{-\lambda_{j} \tau} & (-1) \tau e^{-\lambda_{j} \tau} & \cdots & (-1)^{\left(\mu_{z_{j}}-1\right)} \frac{\tau^{\left(\mu_{z_{j}}-1\right)}}{\left(\mu_{z_{j}}-1\right) !} e^{-\lambda_{j} \tau} \\
0 & e^{-\lambda_{j} \tau} & \cdots & (-1)^{\left(\mu_{z_{j}}-2\right)} \frac{\tau^{\left(\mu_{z_{j}}-2\right)}}{\left(\mu_{z_{j}}-2\right) !} e^{-\lambda_{j} \tau} \\
\vdots & \vdots & \ddots & e^{\lambda_{j} \tau}
\end{array}\right]=\left[\begin{array}{c}
\mathbf{b}_{j z_{j}}^{1} \\
0 \\
0
\end{array}\right] \begin{array}{c}
\mathbf{b}_{j z_{j}}^{2} \\
\vdots \\
\mathbf{b}_{j z_{j}}^{\mu_{j}}
\end{array}\right] u(\tau) d \tau \\
& =\left[\begin{array}{c}
\left.\int_{-\infty}^{t} e^{-\lambda_{j} \tau} \mathbf{b}_{j_{z_{j}}}^{1,} u(\tau) d \tau+\int_{-\infty}^{t}(-1) \tau e^{-\lambda_{j} \tau} \mathbf{b}_{j_{z_{j}}}^{2} u(\tau) d \tau+\cdots+\int_{-\infty}^{t}(-1)^{\left(\mu_{z_{j}}-1\right)} \frac{\tau^{\left(\mu_{z_{j}}-1\right)}}{\left(\mu_{z_{j}}-1\right) !} e^{-\lambda_{j} \tau} \mathbf{b}_{j_{z_{j}}}^{\mu_{j}} u(\tau) d \tau\right] \\
\int_{-\infty}^{t} e^{-\lambda_{j} \tau} \mathbf{b}_{j_{z_{j}}}^{2,} u(\tau) d \tau+\cdots+\int_{-\infty}^{t}(-1)^{\left(\mu_{z_{j}}-2\right)} \frac{\tau^{\left(\mu_{z_{j}}-2\right)}}{\left(\mu_{z_{j}}-2\right) !} \mathbf{b}_{j_{z_{j}}}^{\mu_{j}} u(\tau) d \tau \\
\vdots \\
\int_{-\infty}^{t} e^{-\lambda_{j} \tau} \mathbf{b}_{j_{z_{j}}}^{\mu_{j}} u(\tau) d \tau
\end{array}\right]
\end{aligned}
$$

Remark 6. According to (B.2), in order to able be to calculate the integral $\lim _{\sigma \rightarrow 0} \int_{-\infty}^{t} e^{-J_{i} t} \underline{\mathbf{b}}_{\tau_{i}} u(\tau) d \tau$, we should calculate the $\lim _{\sigma \rightarrow 0} \int_{-\infty}^{t}\left(\tau^{\rho} / \rho !\right) e^{-\lambda \tau} u(\tau) d \tau$.

Lemma 27. One has

$$
\lim _{\sigma \rightarrow 0} \int_{-\infty}^{t} \frac{\tau^{\rho}}{\rho !} e^{-\lambda \tau} u(\tau) d \tau=(-1)^{\rho}\left(\sum_{k=0}^{n-1} \underline{\alpha}_{k} \frac{d^{\rho} \lambda^{k}}{d \lambda^{\rho}} \frac{1}{\rho !}\right) \Phi^{-1}(K) .
$$

However, before we prove Lemma 27, the following lemmas should be considered firstly.

Lemma 28. One has

$$
\lim _{\sigma \rightarrow 0} \int_{-\infty}^{t} \tau e^{-\lambda \tau} u(\tau) d \tau=-\sum_{k=0}^{n-1} \alpha_{k} \frac{d \lambda^{k}}{d \lambda} \Phi^{-1}(K) .
$$

Proof. After some basic calculations, we obtain

$$
\lim _{\sigma \rightarrow 0} \int_{-\infty}^{t} \tau e^{-\lambda \tau} u(\tau) d \tau=\sum_{k=0}^{n-1} \alpha_{k} \lim _{\sigma \rightarrow 0} \int_{-\infty}^{t} \tau e^{-\lambda \tau} \frac{1}{\sigma^{k+1}} \Phi^{(k)}\left(\frac{\tau}{\sigma}\right) d \tau .
$$

First, we calculate

$$
\begin{aligned}
& \lim _{\sigma \rightarrow 0} \int_{-\infty}^{t} \tau e^{-\lambda \tau} \frac{1}{\sigma} \Phi\left(\frac{\tau}{\sigma}\right) d \tau \\
& \quad=\lim _{\sigma \rightarrow 0}\left(\int_{-\infty}^{t} \tau \frac{1}{\sigma} \frac{1}{\sqrt{2 \pi}} e^{-((\tau / \sigma)+\lambda \sigma)^{2} / 2} d \tau \cdot e^{\left(\lambda^{2} \sigma^{2}\right) / 2}\right) .
\end{aligned}
$$

Then $\lim _{\sigma \rightarrow 0} e^{\lambda^{2} \sigma^{2} / 2}=1$ and we obtain $\lim _{\sigma \rightarrow 0} \int_{-\infty}^{t} \tau(1 /$ $\sigma)(1 / \sqrt{2 \pi}) e^{-((\tau / \sigma)+\lambda \sigma)^{2} / 2} d \tau$.

Afterwards, we denote $(\tau / \sigma)+\lambda \sigma=z \Leftrightarrow \tau=\sigma z-$ $\lambda \sigma^{2}$ and $d \tau=\sigma d z$.

And, we take

$$
\begin{aligned}
& \lim _{\sigma \rightarrow 0} \int_{-\infty}^{(t / \sigma)+\lambda \sigma}\left(\sigma z-\lambda \sigma^{2}\right) \frac{1}{\sqrt{2 \pi}} \frac{1}{\sigma} e^{-z^{2} / 2} \sigma d z \\
& \quad=\lim _{\sigma \rightarrow 0}\left[-\frac{\sigma}{\sqrt{2 \pi}} e^{-((t / \sigma)+\lambda \sigma)^{2} / 2}-\sigma^{2} \lambda \Phi^{-1}\left(\frac{t}{\sigma}+\lambda \sigma\right)\right] .
\end{aligned}
$$

In this part, we use also Remark 2.1, with as $t=K \sigma$ with $\sigma \rightarrow 0$ and $K \gg 0$. Thus

$$
\lim _{\sigma \rightarrow 0}\left[-\frac{\sigma}{\sqrt{2 \pi}} e^{-(K+\lambda \sigma)^{2} / 2}-\sigma^{2} \lambda \Phi^{-1}(K+\lambda \sigma)\right]=0 .
$$

Moreover,

$$
\lim _{\sigma \rightarrow 0} \int_{-\infty}^{t} \tau e^{-\lambda \tau} \frac{1}{\sigma} \Phi\left(\frac{\tau}{\sigma}\right) d \tau=0
$$

Now, we calculate

$$
\begin{aligned}
& \lim _{\sigma \rightarrow 0} \int_{-\infty}^{t} \tau e^{-\lambda \tau} \frac{\Phi^{(1)}(\tau / \sigma)}{\sigma^{2}} d \tau\left(\operatorname{denote} \frac{\tau}{\sigma}=z \text {. Then } d \tau=\sigma d z\right) \\
& =\lim _{\sigma \rightarrow 0}\left[\left[z e^{-\lambda \sigma z} \Phi(z)\right]_{-\infty}^{t / \sigma}-\int_{-\infty}^{t / \sigma}\left(e^{-\lambda \sigma z}-\lambda \sigma z e^{-\lambda \sigma z}\right) \Phi(z) d z\right] .
\end{aligned}
$$


The $\lim _{\sigma \rightarrow 0}\left[z e^{-\lambda \sigma z} \Phi(z)\right]_{-\infty}^{t / \sigma}=\lim _{\sigma \rightarrow 0} t / \sigma e^{-\lambda t} \Phi(t / \sigma)=0$, as we have seen (using Remark 2.1).

Then, we obtain

$$
\begin{aligned}
-\lim _{\sigma \rightarrow 0} \int_{-\infty}^{t / \sigma} e^{-\lambda \sigma z} \Phi(z) d z+\lambda \lim _{\sigma \rightarrow 0} \int_{-\infty}^{t / \sigma} \sigma z e^{-\lambda \sigma z} \Phi(z) d z \\
=-\lim _{\sigma \rightarrow 0} \int_{-\infty}^{t} e^{-\lambda \tau} \frac{1}{\sigma} \Phi\left(\frac{\tau}{\sigma}\right) d \tau \\
\quad+\lambda \lim _{\sigma \rightarrow 0} \int_{-\infty}^{t} \tau e^{-\lambda \tau} \frac{1}{\sigma} \Phi\left(\frac{\tau}{\sigma}\right) d \tau=
\end{aligned}
$$

(using (B.9) and (A.10) ) $=-\Phi^{-1}(K)+\lambda \cdot 0=-\Phi^{-1}(\mathrm{~K})$.

Thus, we conclude

$$
\lim _{\sigma \rightarrow 0} \int_{-\infty}^{t} \tau e^{-\lambda \tau} \frac{\Phi^{(1)}(t / \sigma)}{\sigma^{2}} d \tau=-\Phi^{-1}(K) .
$$

Now, we calculate

$$
\begin{aligned}
\lim _{\sigma \rightarrow 0} \int_{-\infty}^{t} \tau e^{-\lambda \tau} \frac{\Phi^{(2)}(\tau / \sigma)}{\sigma^{3}} d \tau \\
=\lim _{\sigma \rightarrow 0}\left(\frac{1}{\sigma}\left[z e^{-\lambda \sigma z} \Phi^{(1)}(z)\right]_{-\infty}^{t / \sigma}\right. \\
\left.\quad-\frac{1}{\sigma} \int_{-\infty}^{t / \sigma}\left(e^{-\lambda \sigma z}-\lambda \sigma z e^{-\lambda \sigma z}\right) \Phi^{(1)}(z) d z\right)
\end{aligned}
$$

(since $\sigma \rightarrow 0$ and $t=K \sigma$, then $\left[z e^{-\lambda \sigma z} \Phi^{(1)}(z)\right]_{-\infty}^{t / \sigma} \rightarrow 0$ ). Thus, we obtain

$$
\begin{aligned}
& \lim _{\sigma \rightarrow 0}\left(-\frac{1}{\sigma} \int_{-\infty}^{t / \sigma} e^{-\lambda \sigma z} \Phi^{(1)}(z) d z+\lambda \frac{1}{\sigma} \int_{-\infty}^{t / \sigma} \sigma z e^{-\lambda \sigma z} \Phi^{(1)}(z) d z\right) \\
& =\lim _{\sigma \rightarrow 0}\left(-\int_{-\infty}^{t} e^{-\lambda \tau} \frac{1}{\sigma^{2}} \Phi^{(1)}\left(\frac{\tau}{\sigma}\right) d \tau+\lambda \int_{-\infty}^{\tau} \tau e^{-\lambda \tau} \frac{1}{\sigma^{2}} \Phi^{(1)}\left(\frac{\tau}{\sigma}\right) d \tau\right)
\end{aligned}
$$

(using (B.9) and (A.12)) $=-\lambda \Phi^{-1}(K)-\lambda \Phi^{-1}(K)=$ $-2 \lambda \Phi^{-1}(K)$.

Consequently,

$$
\lim _{\sigma \rightarrow 0} \int_{-\infty}^{t} \tau e^{-\lambda \tau} \frac{\Phi^{(2)}(\tau / \sigma)}{\sigma^{3}} d \tau=-2 \lambda \Phi^{-1}(K) .
$$

Generalized the above expression, we take

$$
\lim _{\sigma \rightarrow 0} \int_{-\infty}^{t} \tau e^{-\lambda \tau} \frac{\Phi^{(k)}(\tau / \sigma)}{\sigma^{k+1}} d \tau=-\frac{d \lambda^{k}}{d \lambda} \Phi^{-1}(K) .
$$

Now, it should be proved the recursive (B.17)

$$
\lim _{\sigma \rightarrow 0} \int_{-\infty}^{t} \tau e^{-\lambda \tau} \frac{\Phi^{(k+1)}(\tau / \sigma)}{\sigma^{k+2}} d \tau=-\frac{d \lambda^{k+1}}{d \lambda} \Phi^{-1}(K) .
$$

Thus, we obtain

$$
\begin{aligned}
\lim _{\sigma \rightarrow 0} \int_{-\infty}^{t / \sigma} \sigma z e^{-\lambda \sigma z} \frac{\Phi^{(k+1)}(z)}{\sigma^{k+2}} \sigma d z \\
=\lim _{\sigma \rightarrow 0}\left(-\frac{1}{\sigma^{k}} \int_{-\infty}^{t} e^{-\lambda \tau} \frac{\Phi^{(k)}(\tau / \sigma)}{\sigma} d \tau\right. \\
\left.\quad+\lambda \int_{-\infty}^{t} \tau \frac{1}{\sigma^{k+1}} \Phi^{(k)}(\tau) d \tau\right)=
\end{aligned}
$$

(using (B.16) and (A.16) $)=-\left(d \lambda^{k+1} / d \lambda\right) \Phi^{-1}(K)$. The above expression concludes the recursive proof.

Lemma 29. One has

$$
\lim _{\sigma \rightarrow 0} \int_{-\infty}^{t} \frac{\tau^{2}}{2 !} e^{-\lambda \tau} u(\tau) d \tau=(-1)^{2} \frac{1}{2 !}\left(\sum_{k=0}^{n-1} \alpha_{k} \frac{d^{2} \lambda^{k}}{d \lambda^{2}}\right) \Phi^{-1}(K) .
$$

Proof. Consider that

$$
\begin{aligned}
& \lim _{\sigma \rightarrow 0} \int_{-\infty}^{t} \frac{\tau^{2}}{2 !} e^{-\lambda \tau} u(\tau) d \tau \\
& \quad=\sum_{k=0}^{n-1} \alpha_{k} \lim _{\sigma \rightarrow 0} \int_{-\infty}^{t} \frac{\tau^{2}}{2 !} e^{-\lambda \tau} \frac{1}{\sigma^{k+1}} \Phi^{(k)}\left(\frac{\tau}{\sigma}\right) d \tau .
\end{aligned}
$$

First, we calculate

$$
\begin{aligned}
& \lim _{\sigma \rightarrow 0} \int_{-\infty}^{t} \frac{\tau^{2}}{2 !} e^{-\lambda \tau} \frac{1}{\sigma} \Phi\left(\frac{\tau}{\sigma}\right) d \tau \\
& =\lim _{\sigma \rightarrow 0} \int_{-\infty}^{t} \frac{\tau^{2}}{2 !} e^{-\lambda \tau} \frac{1}{\sigma} \frac{1}{\sqrt{2 \pi}} e^{-((\tau / \sigma)+\lambda \sigma)^{2} / 2} d \tau= \\
& \left(\text { Denote } \frac{\tau}{\sigma}+\lambda \sigma=z \Longleftrightarrow \tau=\sigma z-\lambda \sigma^{2}, d \tau=\sigma d z\right) \\
& =\lim _{\sigma \rightarrow 0} \int_{-\infty}^{t / \sigma+\lambda \sigma} \frac{1}{2 !}\left(\sigma z-\lambda \sigma^{2}\right)^{2} \frac{1}{\sigma} \frac{1}{\sqrt{2 \pi}} e^{-\frac{z^{2}}{2}} \sigma d z \\
& =\lim _{\sigma \rightarrow 0}\left(\sigma^{2} \frac{1}{2 !} \int_{-\infty}^{(t / \sigma)+\lambda \sigma} z^{2} \frac{1}{\sqrt{2 \pi}} e^{-z^{2} / 2} d z-\frac{2 \lambda \sigma^{3}}{2 !} \int_{-\infty}^{(t / \sigma)+\lambda \sigma}\right. \\
& \left.z \frac{1}{\sqrt{2 \pi}} e^{-z^{2} / 2} d z+\frac{\lambda^{2} \sigma^{4}}{2 !} \int_{-\infty}^{(t / \sigma)+\lambda \sigma} \frac{1}{\sqrt{2 \pi}} e^{-z^{2} / 2} d z\right) .
\end{aligned}
$$

Following Remark 2.1, we assume that $\sigma \rightarrow 0$ and $t=K \sigma$ with $K \gg 0$. Thus,

$$
\begin{gathered}
\int_{-\infty}^{(t / \sigma)+\lambda \sigma} z^{2} \frac{1}{\sqrt{2 \pi}} e^{-z^{2} / 2} d z \approx 0, \\
\int_{-\infty}^{(t / \sigma)+\lambda \sigma} z \frac{1}{\sqrt{2 \pi}} e^{-z^{2} / 2} d z \approx 0, \\
\int_{-\infty}^{(t / \sigma)+\lambda \sigma} \frac{1}{\sqrt{2 \pi}} e^{-z^{2} / 2} d z \approx \Phi^{-1}(K) .
\end{gathered}
$$


Moreover, we take $\lim _{\sigma \rightarrow 0}\left(\lambda^{2} \sigma^{4} / 2 !\right) \Phi^{-1}(K)=0$. And

$$
\lim _{\sigma \rightarrow 0} \int_{-\infty}^{t} \frac{\tau^{2}}{2 !} e^{-\lambda \tau} \frac{1}{\sigma} \Phi\left(\frac{\tau}{\sigma}\right) d \tau=0 \cdot \Phi^{-1}(K)=0 .
$$

Then, we calculate that

$$
\begin{aligned}
& \lim _{\sigma \rightarrow 0} \int_{-\infty}^{t} \frac{\tau^{2}}{2 !} e^{-\lambda \tau} \frac{1}{\sigma^{2}} \Phi^{(1)}\left(\frac{\tau}{\sigma}\right) d \tau \\
& \quad=\lim _{\sigma \rightarrow 0} \int_{-\infty}^{t / \sigma} \frac{\sigma^{2} z^{2}}{2 !} e^{-\lambda \sigma z} \frac{1}{\sigma^{2}} \Phi^{(1)}(z) \sigma d z \\
& \quad=\lim _{\sigma \rightarrow 0}\left(-\int_{-\infty}^{t} \tau e^{-\lambda \tau} \frac{1}{\sigma} \Phi\left(\frac{\tau}{\sigma}\right) d \tau+\lambda \int_{-\infty}^{t} \tau^{2} e^{-\lambda \tau} \frac{1}{\sigma} \Phi\left(\frac{\tau}{\sigma}\right) d \tau\right)
\end{aligned}
$$

(using (B.9) and (B.23)) $=0$.

Thus, we obtain

$$
\lim _{\sigma \rightarrow 0} \int_{-\infty}^{t} \frac{\tau^{2}}{2 !} e^{-\lambda \tau} \frac{1}{\sigma^{2}} \Phi^{(1)}\left(\frac{\tau}{\sigma}\right) d \tau=0 .
$$

Afterwards, we calculate

$$
\begin{aligned}
\lim _{\sigma \rightarrow 0} \int_{-\infty}^{t} \frac{\tau^{2}}{2 !} e^{-\lambda \tau} \frac{1}{\sigma^{3}} \Phi^{(2)}\left(\frac{\tau}{\sigma}\right) d \tau \\
=\lim _{\sigma \rightarrow 0}\left(-\int_{-\infty}^{t} \tau e^{-\lambda \tau} \frac{1}{\sigma^{2}} \Phi^{(1)}\left(\frac{\tau}{\sigma}\right) d \tau\right. \\
\left.\quad+\frac{1}{2} \lambda \int_{-\infty}^{t} \tau^{2} e^{-\lambda \tau} \frac{1}{\sigma^{2}} \Phi^{(1)}(\tau) d \tau\right)=
\end{aligned}
$$

(using (B.12) and (B.25) $)=-\left(-\Phi^{-1}(K)+0\right)=\Phi^{-1}(K)$.

Hence, we conclude that

$$
\int_{-\infty}^{t} \frac{\tau^{2}}{2 !} e^{-\lambda \tau} \frac{1}{\sigma^{3}} \Phi^{(2)}\left(\frac{\tau}{\sigma}\right) d \tau=\Phi^{-1}(K) .
$$

Now, we calculate that

$$
\begin{aligned}
\lim _{\sigma \rightarrow 0} \int_{-\infty}^{t} \frac{\tau^{2}}{2 !} e^{-\lambda \tau} \frac{1}{\sigma^{4}} \Phi^{(3)}\left(\frac{\tau}{\sigma}\right) d \tau & \\
=\lim _{\sigma \rightarrow 0}( & -\int_{-\infty}^{t} \tau e^{-\lambda \tau} \frac{1}{\sigma^{3}} \Phi^{(2)}\left(\frac{\tau}{\sigma}\right) d \tau \\
& \left.\quad+\frac{1}{2} \lambda \int_{-\infty}^{t} \tau^{2} e^{-\lambda \tau} \frac{1}{\sigma^{3}} \Phi^{(2)}\left(\frac{\tau}{\sigma}\right) d \tau\right)=
\end{aligned}
$$

(using (B.15) and (B.27)) $=-\left(-2 \lambda \Phi^{-1}(K)\right)+\lambda \Phi^{-1}(K)=$ $3 \lambda \Phi^{-1}(K)$.

Hence, we obtain

$$
\lim _{\sigma \rightarrow 0} \int_{-\infty}^{t} \frac{\tau^{2}}{2 !} e^{-\lambda \tau} \frac{1}{\sigma^{4}} \Phi^{(3)}\left(\frac{\tau}{\sigma}\right) d \tau=3 \lambda \Phi^{-1}(K) .
$$

Combining the equations (B.23)-(B.29), we obtain recursively (B.30)

$$
\lim _{\sigma \rightarrow 0} \int_{-\infty}^{t} \frac{\tau^{2}}{2 !} e^{-\lambda \tau} \frac{1}{\sigma^{k+1}} \Phi^{(k)}\left(\frac{\tau}{\sigma}\right) d \tau=\frac{1}{2 !} \frac{d^{2} \lambda^{k}}{d \lambda^{2}} \Phi^{-1}(K) .
$$

Thus, in order to conclude, we should prove

$$
\lim _{\sigma \rightarrow 0} \int_{-\infty}^{t} \frac{\tau^{2}}{2 !} e^{-\lambda \tau} \frac{1}{\sigma^{k+2}} \Phi^{(k+1)}\left(\frac{\tau}{\sigma}\right) d \tau=\frac{1}{2 !} \frac{d^{2} \lambda^{k+1}}{d \lambda^{2}} \Phi^{-1}(K) .
$$

Hence,

$$
\begin{aligned}
\lim _{\sigma \rightarrow 0} \int_{-\infty}^{t} \frac{\tau^{2}}{2 !} e^{-\lambda \tau} \frac{1}{\sigma^{k+2}} \Phi^{(k+1)}\left(\frac{\tau}{\sigma}\right) d \tau \\
=\lim _{\sigma \rightarrow 0} \frac{1}{2 !} \frac{1}{\sigma^{k-1}} \int_{-\infty}^{t / \sigma} z^{2} e^{-\lambda \sigma z} \Phi^{(k+1)}(z) d z \\
=\lim _{\sigma \rightarrow 0}\left(-\int_{-\infty}^{t} \tau e^{-\lambda \tau} \frac{1}{\sigma^{k+1}} \Phi^{(k)}\left(\frac{\tau}{\sigma}\right) d \tau\right. \\
\left.+\lambda \int_{-\infty}^{t} \frac{\tau^{2}}{2 !} e^{-\lambda \tau} \frac{1}{\sigma^{k+1}} \Phi^{(k)}\left(\frac{\tau}{\sigma}\right) d \tau\right)
\end{aligned}
$$

(using (B.16) and (B.30)) $=((k+1) k / 2 !) \lambda^{k-1} \Phi^{-1}(K)=$ $(1 / 2 !)\left(d^{2} \lambda^{k+1} / d \lambda^{2}\right) \Phi^{-1}(K)$.

The proof is concluded by considering.

Lemma 30. One has

$$
\lim _{\sigma \rightarrow 0} \int_{-\infty}^{t} \frac{\tau^{\rho}}{\rho !} e^{-\lambda \tau} \frac{1}{\sigma^{k+1}} \Phi^{(k)}\left(\frac{\tau}{\sigma}\right) d \tau=(-1)^{\rho} \frac{1}{\rho !} \frac{d^{\rho} \lambda^{k}}{d \lambda^{\rho}} \Phi^{-1}(K) .
$$

Proof. For $\rho=1$, we have proved (B.16).

For $\rho=2$, we have also proved (B.30).

We assume that (B.33) is true.

Now, we easily have to prove the following expression for $\rho+1$, that is,

$$
\begin{array}{r}
\lim _{\sigma \rightarrow 0} \int_{-\infty}^{t} \frac{\tau^{\rho+1}}{(\rho+1) !} e^{-\lambda \tau} \frac{1}{\sigma^{k+1}} \Phi^{(k)}\left(\frac{\tau}{\sigma}\right) d \tau \\
=(-1)^{\rho+1} \frac{1}{(\rho+1) !} \frac{d^{\rho+1} \lambda^{k}}{d \lambda^{\rho+1}} \Phi^{-1}(K) .
\end{array}
$$

Now, we return to the proof of Lemma 27.

Proof. Consider that

$$
\begin{aligned}
\lim _{\sigma \rightarrow 0} \int_{-\infty}^{t} \frac{\tau^{\rho}}{\rho !} e^{-\lambda \tau} u(\tau) d \tau \\
\quad=\sum_{k=0}^{n-1} \alpha_{k} \lim _{\sigma \rightarrow 0} \int_{-\infty}^{t} \frac{\tau^{\rho}}{\rho !} e^{-\lambda \tau} \frac{1}{\sigma^{k+1}} \Phi^{(k)}\left(\frac{\tau}{\sigma}\right) d \tau .
\end{aligned}
$$

Then,

$$
\begin{aligned}
\lim _{\sigma \rightarrow 0} \int_{-\infty}^{t} \frac{\tau^{\rho}}{\rho !} e^{-\lambda \tau} u(\tau) d \tau \\
\quad=\sum_{k=0}^{n-1} \alpha_{k} \cdot \lim _{\sigma \rightarrow 0} \int_{-\infty}^{t} \frac{\tau^{\rho}}{\rho !} e^{-\lambda \tau} \frac{1}{\sigma^{k+1}} \Phi^{(k)}\left(\frac{\tau}{\sigma}\right) d \tau
\end{aligned}
$$


(considering (B.33))

$$
\begin{aligned}
& =\sum_{k=0}^{n-1} \alpha_{k}(-1)^{\rho} \frac{1}{\rho !} \frac{d^{\rho} \lambda^{k}}{d \lambda^{\rho}} \Phi^{-1}(K) \\
& =(-1)^{\rho} \frac{1}{\rho !} \Phi^{-1}(K)\left(\sum_{k=0}^{n-1} \frac{1}{\rho !} \frac{d^{\rho} \lambda^{k}}{d \lambda^{\rho}}\right) .
\end{aligned}
$$

Remark 7. In all the proofs of Lemma 27-30, we have used

$$
\lim _{\sigma \rightarrow 0}\left[z^{\rho} e^{-\lambda \sigma z} \Phi^{(k)}(z)\right]_{-\infty}^{t / \sigma}=0
$$

for every $\kappa$ and every $\rho$. In this part of Appendix B, we return to expression (B.2) and we calculate

$$
\lim _{\sigma \rightarrow 0} \int_{-\infty}^{t} e^{-J_{j_{j}} \tau} \underline{\mathbf{b}}_{j_{z_{j}}} u(\tau) d \tau=
$$

(using (A.23) and (B.3), and calculating the limit as $\sigma \rightarrow 0$, we obtain what follows)

$$
\begin{aligned}
& \lim _{\sigma \rightarrow 0}\left[\begin{array}{c}
\int_{-\infty}^{t} e^{-\lambda_{j} \tau} \sum_{c=1}^{\mu_{z_{j}}} \mathbf{b}_{j_{z_{j}}}^{c}(-1)^{c-1} \frac{\tau^{c-1}}{(c-1) !} u(\tau) d \tau \\
\int_{-\infty}^{t} e^{-\lambda_{j} \tau} \sum_{c=2}^{\mu_{z_{j}}} \mathbf{b}_{j_{z_{j}}}(-1)^{c-2} \frac{\tau^{c-2}}{(c-2) !} u(\tau) d \tau \\
\vdots \\
\int_{-\infty}^{t} e^{-\lambda_{j} \tau} \sum_{c=\mu_{z j}}^{\mu_{z_{j}}} \mathbf{b}_{j_{z_{j}}}^{c}(-1)^{c-\mu_{z j}} \frac{\tau^{c-\mu_{z j}}}{\left(c-\mu_{z j}\right) !} u(\tau) d \tau
\end{array}\right] \\
& =\left[\begin{array}{c}
\sum_{c=1}^{\mu_{z_{j}}} \mathbf{b}_{j_{z_{j}}}^{c}(-1)^{c-1} \cdot \lim _{\sigma \rightarrow 0} \int_{-\infty}^{t} e^{-\lambda_{j} \tau} \frac{\tau^{c-1}}{(c-1) !} u(\tau) d \tau \\
\sum_{c=2}^{\mu_{z_{j}}} \mathbf{b}_{j_{z_{j}}}^{c}(-1)^{c-2} \cdot \lim _{\sigma \rightarrow 0} \int_{-\infty}^{t} e^{-\lambda_{j} \tau} \frac{\tau^{c-2}}{(c-2) !} u(\tau) d \tau \\
\vdots \\
\sum_{c=\mu_{z_{j}}} \mathbf{b}_{j_{z_{j}}}^{c}(-1)^{c-\mu_{z_{j}}} \cdot \lim _{\sigma \rightarrow 0} \int_{-\infty}^{t} e^{-\lambda_{j} \tau} \frac{\tau^{c-\mu_{z_{j}}}}{\left(c-\mu_{z_{j}}\right) !} u(\tau) d \tau
\end{array}\right] \\
& =\left[\begin{array}{c}
\sum_{c=1}^{\mu_{z_{j}}} \mathbf{b}_{j_{z_{j}}}^{c}(-1)^{2(c-1)} \cdot \frac{1}{(c-1) !}\left(\sum_{k=0}^{n-1} \alpha_{k} \frac{d^{c-1} \lambda_{j}^{k}}{d \lambda_{j}^{c-1}}\right) \Phi^{-1}(K) \\
\sum_{c=2}^{\mu_{z_{j}}} \mathbf{b}_{j_{z_{j}}}^{c}(-1)^{2(c-2)} \cdot \frac{1}{(c-2) !}\left(\sum_{k=0}^{n-1} \alpha_{k} \frac{d^{c-2} \lambda_{j}^{k}}{d \lambda_{j}^{c-2}}\right) \Phi^{-1}(K) \\
\vdots \\
\sum_{c=\mu_{z_{j}}} \mathbf{b}_{j_{z_{j}}}^{c}(-1)^{2\left(c-\mu_{z_{j}}\right)} \cdot \frac{1}{\left(c-\mu_{z_{j}}\right) !}\left(\sum_{k=0}^{n-1} \alpha_{k} \frac{d^{c-\mu_{z_{j}}} \lambda_{j}^{k}}{d \lambda_{j}^{c-\mu_{z_{j}}}}\right) \Phi^{-1}(K)
\end{array}\right]
\end{aligned}
$$

Consequently, we obtain

$$
\begin{aligned}
\lim _{\sigma \rightarrow 0} \int_{-\infty}^{t} e^{-J_{z_{j}} \tau} \underline{\mathbf{b}}_{j_{z_{j}}} u(\tau) d \tau & {\left[\begin{array}{c}
\sum_{c=1}^{\mu_{z_{j}}} \mathbf{b}_{j_{z_{j}}} \frac{1}{(c-1) !} \sum_{k=0}^{n-1} \alpha_{k} \frac{d^{c-1} \lambda_{j}^{k}}{d \lambda_{j}^{c-1}} \\
\sum_{z_{j}} \mathbf{b}_{j_{z_{j}}}^{c,} \frac{1}{(c-2) !} \sum_{k=0}^{n-1} \alpha_{k} \frac{d^{c-2} \lambda_{j}^{k}}{d \lambda_{j}^{c-2}} \\
\vdots \\
\sum_{c=\mu_{z_{j}}} \mathbf{b}_{j_{z_{j}}}^{c} \frac{1}{\left(c-\mu_{z_{j}}\right) !} \sum_{k=0}^{n-1} \alpha_{k} \frac{d^{c-\mu_{z_{j}}} \lambda_{j}^{k}}{d \lambda_{j}^{c-\mu_{z_{j}}}}
\end{array}\right] . }
\end{aligned}
$$

Combining (B.1) and (B.41), we obtain (B.42) for every $j=$ $l+1, l+2, \ldots, k$, that is,

$$
\lim _{\sigma \rightarrow 0} \int_{-\infty}^{t} e^{-J_{j} t} \underline{\mathbf{b}}_{\tau_{j}} u(\tau) d \tau
$$

$$
=\left[\begin{array}{c}
\int_{-\infty}^{t} e^{-J_{j_{1}} \tau} \underline{\mathbf{b}}_{j_{1}} u(\tau) d \tau \\
\int_{-\infty}^{t} e^{-J_{j_{2}} \tau} \underline{\mathbf{b}}_{j_{2}} u(\tau) d \tau \\
\vdots \\
\int_{-\infty}^{t} e^{-J_{j_{j}} \tau} \underline{\mathbf{b}}_{d_{d_{j}}} u(\tau) d \tau
\end{array}\right]
$$$$
=\Phi^{-1}(K)\left[\begin{array}{c}
\sum_{c=1}^{\mu_{1}} \mathbf{b}_{j_{1}}^{c} \frac{1}{(c-1) !} \sum_{k=0}^{n-1} \alpha_{k} \frac{d^{c-1} \lambda_{j}^{k}}{d \lambda_{j}^{c-1}} \\
\sum_{c=2}^{\mu_{1}} \mathbf{b}_{j_{1}}^{c} \frac{1}{(c-2) !} \sum_{k=0}^{n-1} \alpha_{k} \frac{d^{c-2} \lambda_{j}^{k}}{d \lambda_{j}^{c-2}} \\
\vdots \\
\sum_{c=\mu_{1}}^{\mu_{1}} \mathbf{b}_{j_{1}}^{c} \frac{1}{\left(c-\mu_{1}\right) !} \sum_{k=0}^{n-1} \alpha_{k} \frac{d^{c-\mu_{1}} \lambda_{j}^{k}}{d \lambda_{j}^{c-\mu_{1}}}
\end{array}\right\} \mu_{1} \times 1
$$ 
where $\mu_{z_{j}}$ are the Weyr characteristics via Ferrer diagrams, for $j=l+1, l+2, \ldots, \kappa$ and $z_{j}=1,2, \ldots, d_{j}$. Note that $\rho_{j}=\max _{z_{i}=1,2, \ldots, d_{j}} \mu_{z_{j}}$ is the index of annihila- tion for the eigenvalue $\lambda_{j}$. Note that matrix (B.42) has $d_{j}$-blocks. Each one of the $d_{j}$ blocks can be written as follows:

$$
\begin{aligned}
& \Phi^{-1}(K)\left[\begin{array}{c}
\sum_{c=1}^{\mu_{z_{j}}} \mathbf{b}_{j_{z_{j}}}^{c} \frac{1}{(c-1) !} \sum_{k=0}^{n-1} \alpha_{k} \frac{d^{c-1} \lambda_{j}^{k}}{d \lambda_{j}^{c-1}} \\
\sum_{c=2}^{\mu_{z_{j}}} \mathbf{b}_{j_{z_{j}}}^{c} \frac{1}{(c-2) !} \sum_{k=0}^{n-1} \alpha_{k} \frac{d^{c-2} \lambda_{j}^{k}}{d \lambda_{j}^{c-2}} \\
\vdots \\
\sum_{c=\mu_{z_{j}}}^{\mu_{z_{j}}} \mathbf{b}_{j_{z_{j}}}^{c} \frac{1}{\left(c-\mu_{2}\right) !} \sum_{k=0}^{n-1} \alpha_{k} \frac{d^{c-\mu_{z_{j}}} \lambda_{j}^{k}}{d \lambda_{j}^{c-\mu_{z_{j}}}}
\end{array}\right]
\end{aligned}
$$

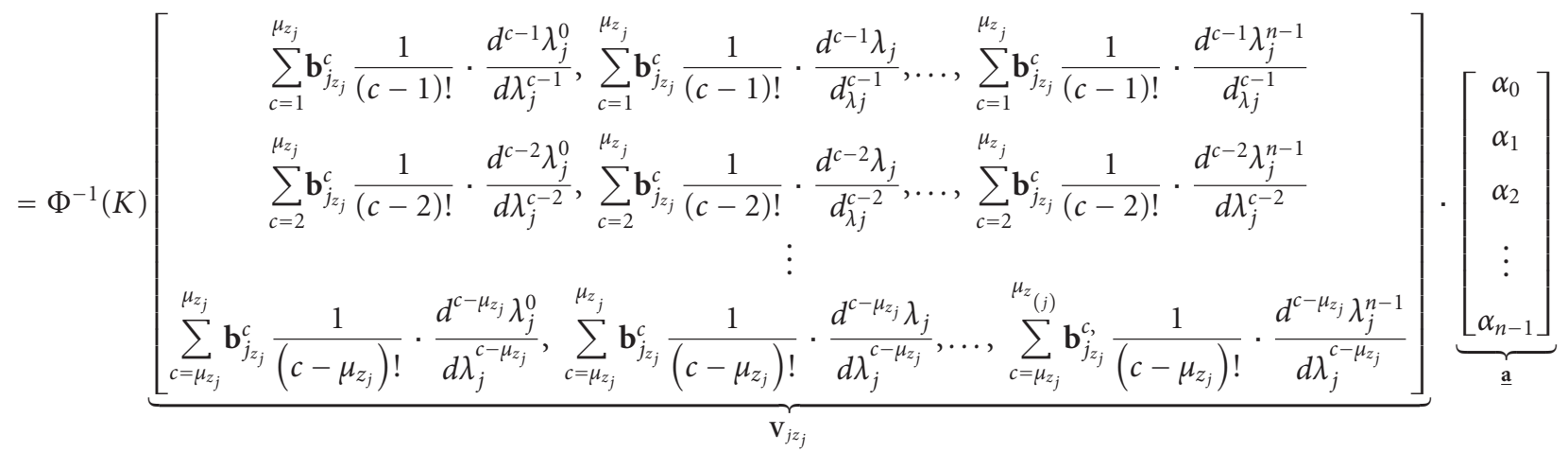

$$
\begin{aligned}
& =\Phi^{-1}(K) \mathbf{V}_{j_{j}} \underline{\mathbf{a}},
\end{aligned}
$$

for every $j=l+1, l+2, \ldots, \kappa$, and $z_{j}=1,2, \ldots, d_{j}$.

Consequently, the matrix (B.42), by using (B.43), can be written as follows

$$
\lim _{\sigma \rightarrow 0} \int_{-\infty}^{t} e^{-J_{j} t} \underline{\mathbf{b}}_{\tau_{j}} u(\tau) d \tau=\Phi^{-1}(K)\left[\begin{array}{c}
\mathbf{V}_{j_{1}} \\
\hline \mathbf{V}_{j_{2}} \\
\hline \vdots \\
\hline \\
\hline \mathbf{V}_{j_{j}} \\
\hline \vdots \\
\hline \mathbf{V}_{j_{j}} \\
\hline
\end{array}\right] \cdot \underline{\mathbf{a}}
$$

\section{Acknowledgments}

The authors are very grateful to Editor Professor Amit Bhaya and the anonymous referees for their comments and suggestions, which highly improved the quality of the paper. The second author is also very grateful to Mr. Aristomenis Tsiomos, Mr. Chris Andrianoupolitis, Ms. Maria Kelertzi, Ms. Aggeliki Drakopoulou, and Mr. Michael Filakouris for their important moral support.

\section{References}

[1] S. C. Gupta and L. Hasdorff, "Changing the state of a linear system by use of normal function and its derivatives," Journal of Electronics and Control, vol. 14, pp. 351-359, 1963.

[2] S. C. Gupta, Transform and State Variable Methods in Linear Systems, John Wiley \& Sons, New York, NY, USA, 1966.

[3] N. Karcanias and B. Kouvaritakis, "Zero time adjustment of initial conditions and its relationship to controllability subspaces," International Journal of Control, vol. 29, no. 5, pp. 749-765, 1979.

[4] G. I. Kalogeropoulos, A. D. Karageorgos, and A. A. Pantelous, "Changing the state of a linear differential system in (almost) zero time by using distributional input function," Systems Science, vol. 33, no. 4, pp. 37-56, 2007.

[5] G. I. Kalogeropoulos, A. D. Karageogos, and A. A. Pantelous, "Changing the state of a generalized regular differential system in (almost) zero time," Journal of Institute of Mathematics and Computer Sciences. Mathematics Series, vol. 20, no. 2, pp. 93112, 2007.

[6] A. A. Pantelous, A. D. Karageorgos, and G. I. Kalogeropoulos, "Some remarks for the generalized inverses of the Vandermonde matrix," Tech. Rep. 09-01-02, University of Athens, Athens, Greece.

[7] A. A. Pantelous, A. D. Karageorgos, and G. I. Kalogeropoulos, "Approximating distributional behaviour, systems theory and control," in Proceedings of the 6th Vienna International Conference on Mathematical Modelling (MathMod '09), I. Troch 
and F. Breitenecker, Eds., Series ARGESIM Reports no. 35, pp. 2246-2256, 2009.

[8] S. L. Campbell, Singular Systems of Differential Equations, vol. 1, Pitman, San Francisco, Calif, USA, 1980.

[9] S. L. Campbell, Singular Systems of Differential Equations, vol. 2, Pitman, San Francisco, Calif, USA, 1982.

[10] R. F. Gantmacher, The Theory of Matrices, Vol. I and II, Chelsea, New York, NY, USA, 1959.

[11] F. Lewis, "A survey of linear descriptor systems," Circuits Systems Signal Process, vol. 5, no. 1, pp. 3-36, 1986.

[12] J. M. Bowen, "Delta function terms arising from classical point source fields," American Journal of Physics, vol. 62, pp. 511515, 1994.

[13] T. B. Boykin, "Derivatives of the Dirac delta function by explicit construction of sequences," American Journal of Physics, vol. 71, no. 5, pp. 462-468, 2003.

[14] R. Estrada and R. P. Kanwal, Singular Integral Equations, Birkhäuser, Boston, Mass, USA, 2000.

[15] R. P. Kanwal, Generalized Functions: Theory and Applications, Birkhäuser, Boston, Mass, USA, 3rd edition, 2004.

[16] A. H. Zemanian, Distribution Theory and Transform Analysis: An Introduction to Generalized Functions with Applications, Dover, New York, NY, USA, 1987.

[17] L. A. Zadeh and C. A. Desoer, Linear System Theory, the State Space Approach, McGraw-Hill, New York, NY, USA.

[18] G. I. Kalogeropoulos, A. D. Karageorgos, and A. A. Pantelous, "Higher-order linear matrix descriptor differential equations of apostol-kolodner type," Electronic Journal of Differential Equations, vol. 2009, no. 25, pp. 1-13, 2009.

[19] A. Ben-Israel and T. N. E. Greville, Generalized Inverses: Theory and Applications, John Wiley \& Sons, New York, NY, USA, 1974.

[20] A. Bjerhammar, "A generalized matrix algebra," Kungliga Tekniska Högskolan Handlingar, vol. 124, pp. 1-32, 1968.

[21] B. N. Datta, Numerical Methods for Linear Control Systems Design and Analysis, ITP, 2003. 

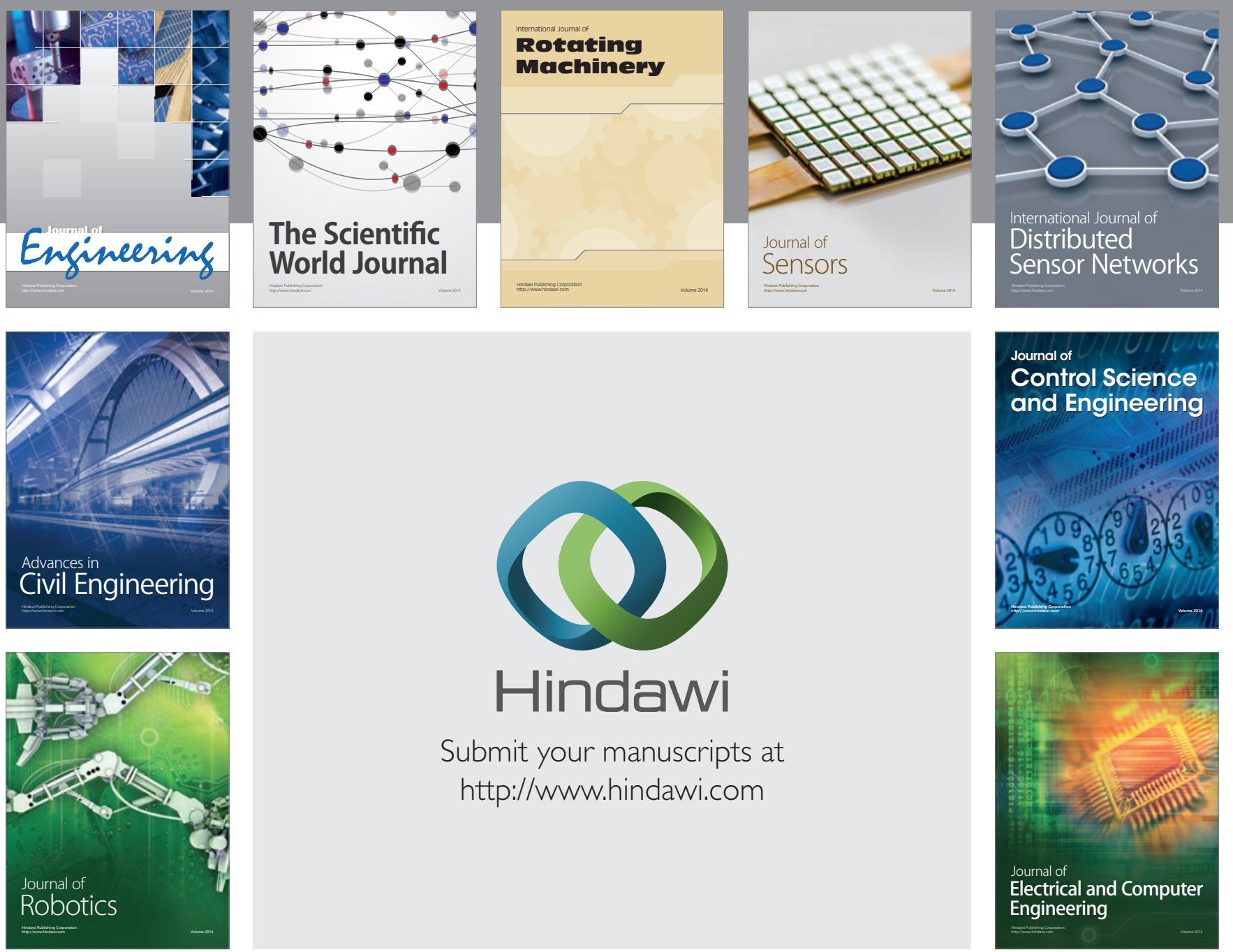

Submit your manuscripts at

http://www.hindawi.com
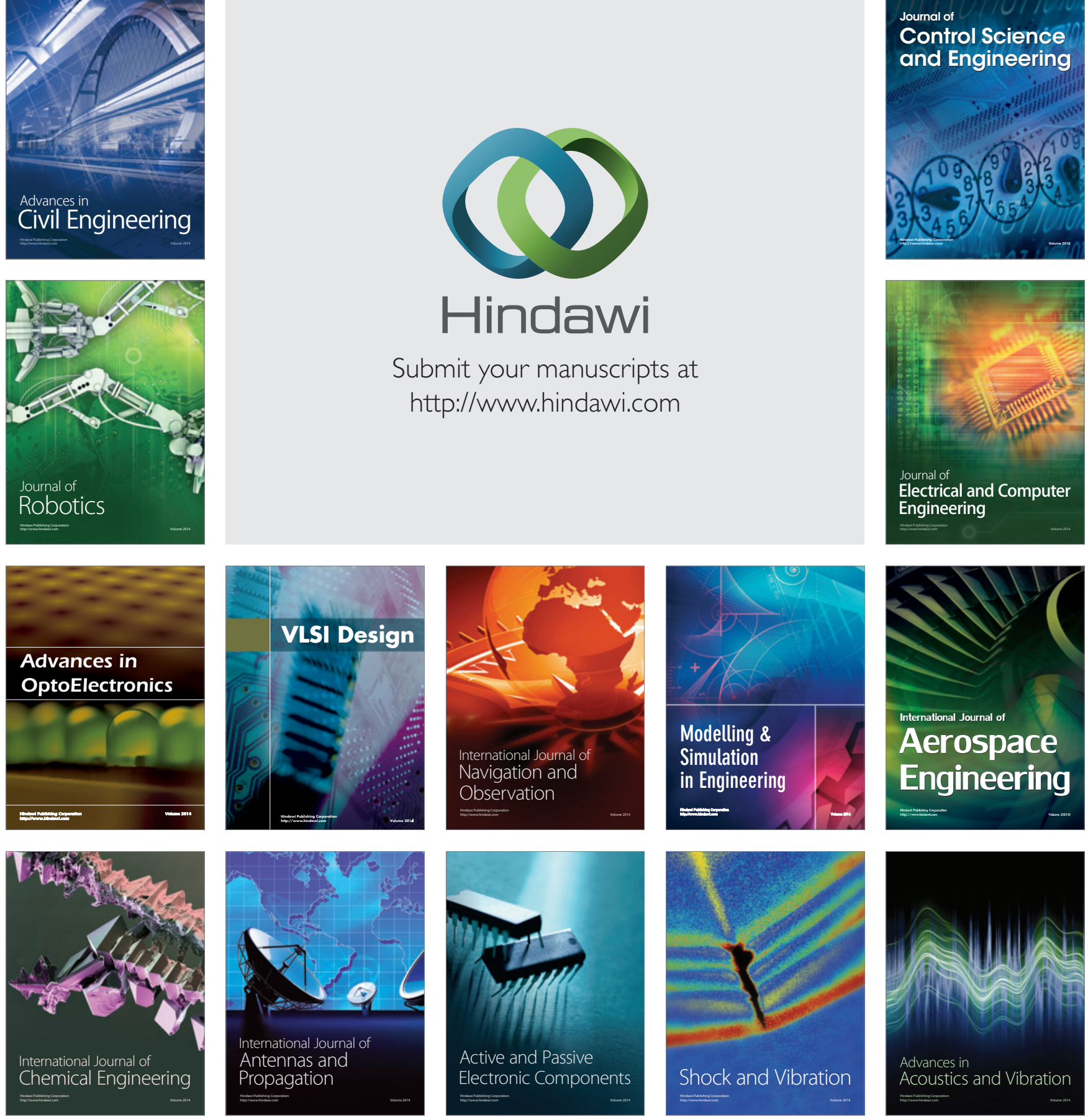